\title{
SYNTHESIS AND CHARACTERIZATION OF CDSE/ZNS CORE/SHELL QUANTUM DOT SENSITIZED PCPDTBT-P3HT:PCBM ORGANIC PHOTOVOLTAICS
}

\author{
A Thesis \\ presented to \\ the Faculty of California Polytechnic State University, \\ San Luis Obispo
}

\author{
In Partial Fulfillment \\ of the Requirements for the Degree
}

Master of Science in Engineering with Specializations in Materials Engineering

by

Buddy James Bump

July 2014 
(C) 2014

Buddy James Bump ALL RIGHTS RESERVED 


\section{COMMITTEE MEMBERSHIP}

TITLE:

Synthesis and Characterization of CdSe/ZnS Core/Shell Quantum Dot Sensitized PCPDTBT-P3HT:PCBM Organic Photovoltaics

AUTHOR:

Buddy James Bump

DATE SUBMITTED:

July 2014

COMMITTEE CHAIR: $\quad$ Richard Savage, Ph.D.

Professor of Materials Engineering

COMMITTEE MEMBER: $\quad$ Robert Echols, Ph.D.

Professor of Physics

COMMITTEE MEMBER: $\quad$ Linda Vanasupa, Ph.D.

Professor of Materials Engineering 


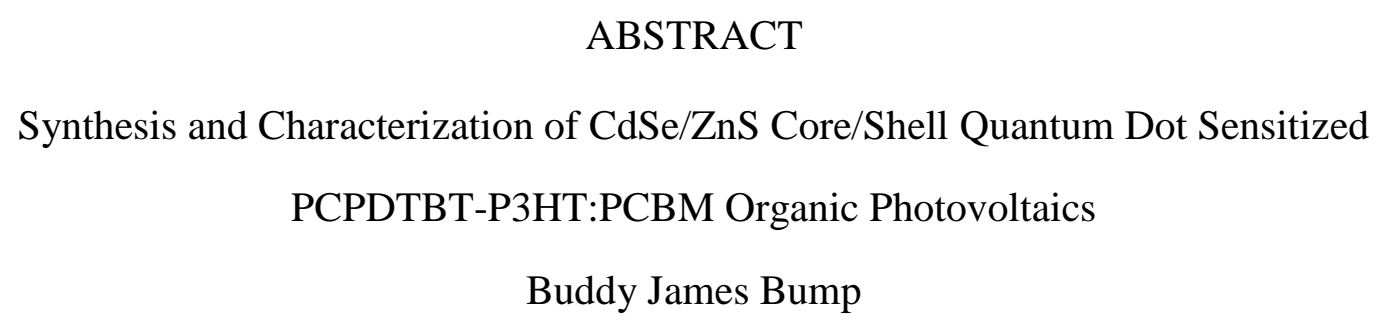

Durable, cheap, and lightweight polymer based solar cells are needed, if simply to meet the demand for decentralized electrical power production in traditionally "off-grid" areas. Using a blend of Poly(3-hexylthiophene-2,5-diyl) (P3HT), Phenyl-C61-butyric acid methyl ester (PCBM), and the low band-gap polymer Poly[2,6-(4,4-bis-(2ethylhexyl)-4H-cyclopenta [2,1-b;3,4-b']dithiophene)-alt-4,7(2,1,3-benzothiadiazole)] (PCPDTBT), we have fabricated devices with a wide spectral response and 3\% power conversion efficiency in AM 1.5 conditions; however, this thin film system exhibits only 0.43 optical density at $500 \mathrm{~nm}$. To improve the performance of this polymer blend photovoltaic, we aim to increase absorption by adding $\mathrm{CdSe}(\mathrm{ZnS})$ core (shell) quantum dots. Four groups of devices are fabricated: a control group with an active polymer layer of $16 \mathrm{mg} / \mathrm{mL}$ P3HT, $16 \mathrm{mg} / \mathrm{mL}$ PCBM, and $4 \mathrm{mg} / \mathrm{mL}$ PCPDTBT; and three groups with dispersed quantum dots at $4 \mathrm{mg} / \mathrm{ml}, 1 \mathrm{mg} / \mathrm{mL}$, and $0.25 \mathrm{mg} / \mathrm{mL}$. The $(\mathrm{CdSe}) \mathrm{ZnS}$ quantum dots are coated with octadecylamine ligands and have a peak absorbance at $560 \mathrm{~nm}$ and peak emission at $577 \mathrm{~nm}$. The active layer was dissolved in chlorobenzene solvent and spun on glass substrates, patterned with indium tin oxide. The devices were then annealed for fifteen minutes at $110^{\circ} \mathrm{C}, 140^{\circ} \mathrm{C}$, and $170^{\circ} \mathrm{C}$. Current-voltage characteristic curves $\mathrm{V}$

and optical density data were taken before and after the anneal step. Finally, surface characterization was conducted with atomic force microscopy and electrostatic force microscopy. When compared to the control, the sensitized devices exhibited increased absorption and depressed electrical performance with increasing quantum dot loading. The surface morphology, both electrical and physical, showed deviation from the typical 
values and patterns shown by the control that increased with quantum dot loading. When the degrading electrical characteristics, increasing optical absorbance, and surface changes, are considered together, it becomes likely that the quantum dots interact in a significant manner with the morphology of the P3HT phase, which leads to an overall decrease in performance.

Keywords: P3HT PCBM OPVs, PCPDTBT low band gap polymer, $\mathrm{CdSe}(\mathrm{ZnS})$ quantum dots, and wide spectral response 


\section{ACKNOWLEDGMENTS}

I would like to acknowledge everyone who helped me along this journey through the world of academia. When I consider who I was when I set out to accomplish this goal, and who I am now, I know I am the better for it. Specifically, I would like to thank Prof. Richard Savage, for his support and guidance; Prof. David Braun, for allowing me to work in the polymer electronics laboratory; Prof. Heather Smith, for her help with the statistical details; Prof. Linda Vanasupa, for her guidance and personal counsel; Grant Olson, for his collaboration throughout the project; Ross Gregoriev, for his skill with AFM and EFM imaging; and Prof. Robert Echols for his sustained and continued support throughout the project.

Lastly, I would like to thank the reader, because you give these pages meaning. 


\section{TABLE OF CONTENTS}

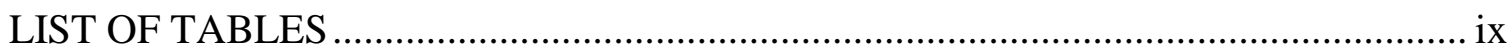

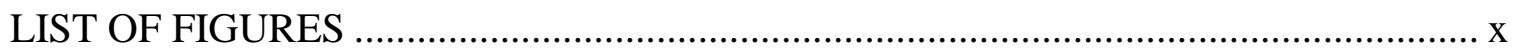

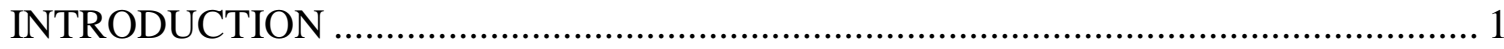

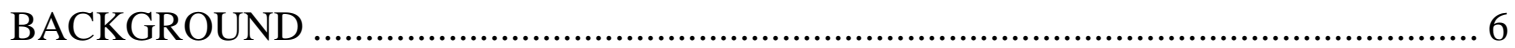

Semiconducting Polymers and the Bulk Heterojunction ............................................ 12

Creating Free Charge Carriers................................................................................ 16

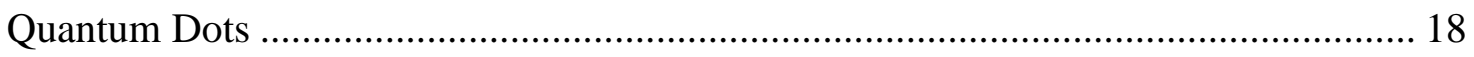

Solar Cell Characterization ............................................................................ 19

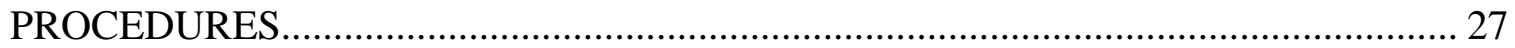

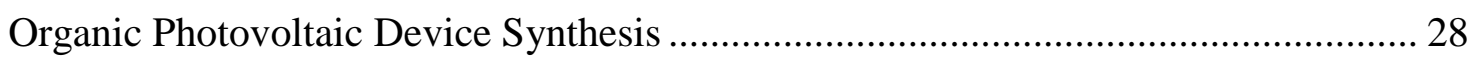

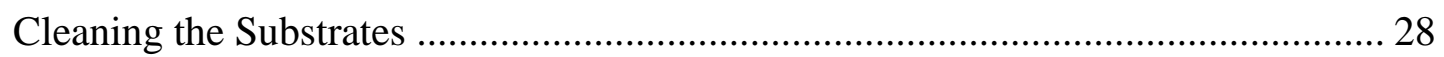

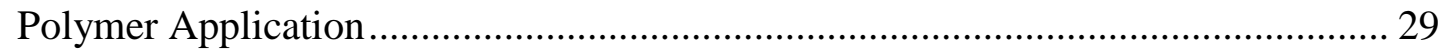

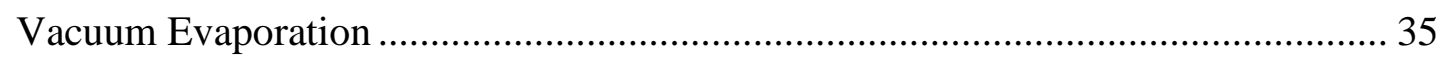

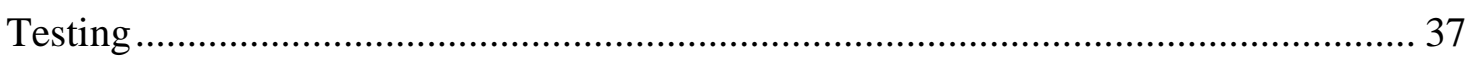

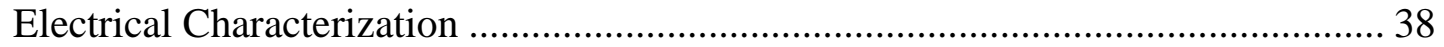

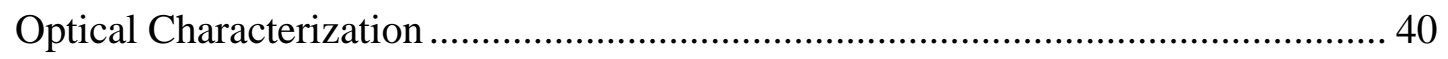

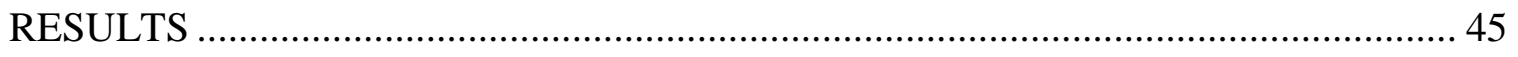

Annealed Devices.......................................................................................... 49

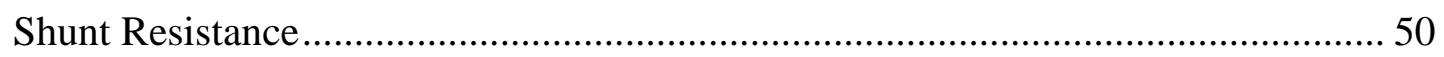

Short Circuit Current Density ..................................................................................... 51

Open Circuit Voltage ........................................................................................... 53

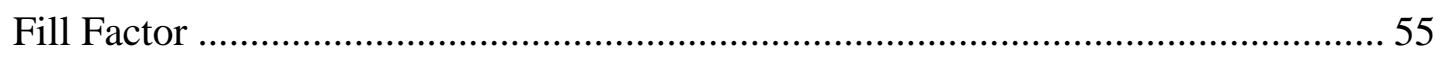

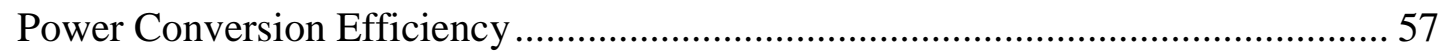

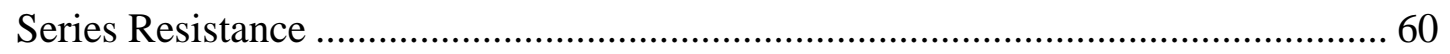

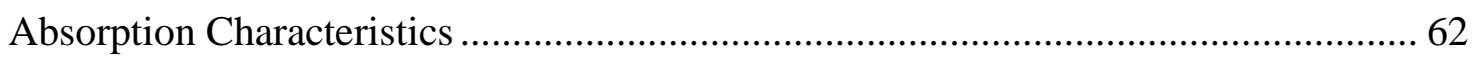

Comparison Between Anneal Temperatures ............................................................ 63

Comparison of Quantum Dot Loading Over Anneal Temperatures............................ 67

As-Cast and Post Anneal Comparison .................................................................... 72 
Short Circuit Current Density ................................................................................. 74

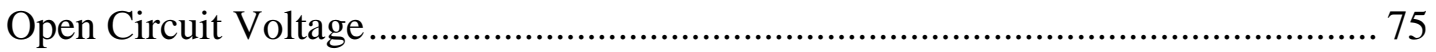

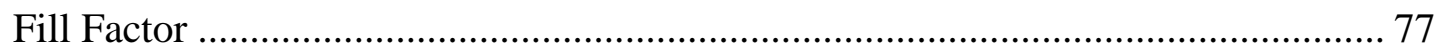

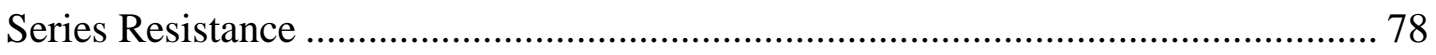

Atomic Force Microscopy and Electrostatic Force Microscopy..................................... 78

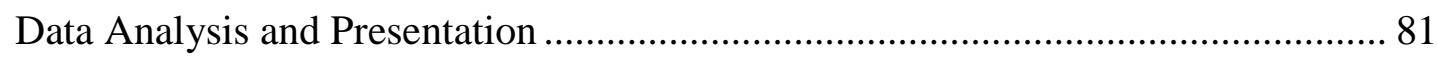

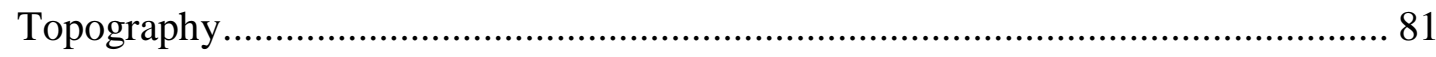

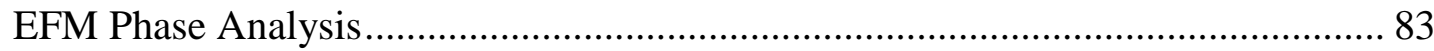

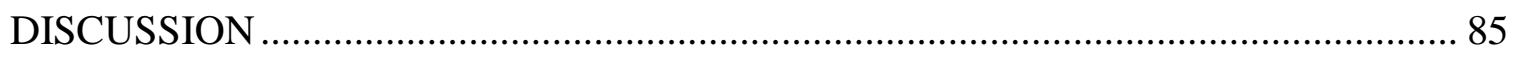

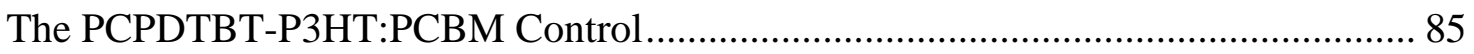

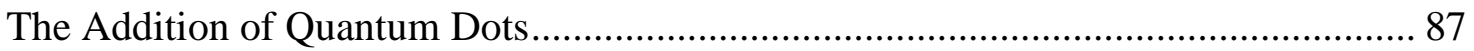

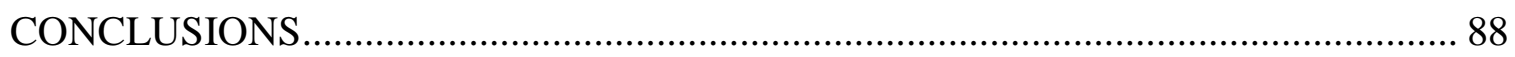

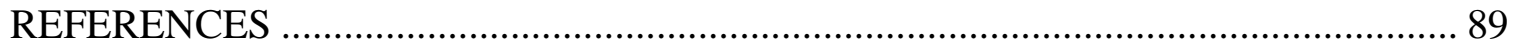




\section{LIST OF TABLES}

Table I: Visualization of Experimental Layout ...................................................... 27

Table II: F-Statistic Values for Statistically Significant Effects on Each Response ........ 49

Table III: Test Statistics and P-Values for Short Circuit Current Density ...................... 52

Table IV: Test statistics and P-Values for Open Circuit Voltage................................. 54

Table V: Test statistics and P-Values for Fill Factor............................................... 56

Table VI: Test statistic and P-Values for Power Conversion Efficiency........................ 58

Table VII: Test statistics and P-Values for Series Resistance .................................... 61

Table VIII: Quantum Dot Sensitized Cells Compared to the PCPDTBT-P3HT:PCBM

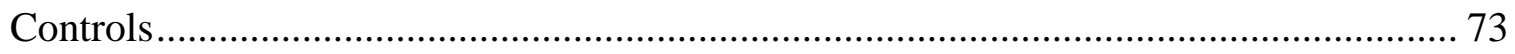




\section{LIST OF FIGURES}

Figure 1: ASTM G173 standard solar spectral emission at $1000 \mathrm{~W} /\left(\mathrm{m}^{2} \mathrm{~nm}\right)$.

Figure 2: Schematic representation of the concept behind the air mass measurement, showing the tilt away from zenith required for AM 1.5 and AM 2.0...... 8

Figure 3: Focused view of AM 1.5G expressed as energy per wavelength (black) and photon flux (red). The standard AM 1.5G spectrum extends from $280 \mathrm{~nm}$ to $4000 \mathrm{~nm}$. . 10 Figure 4: Spectral power density, the amount of power collected at any one wavelength, and spectral current density, the number of photons falling at any one wavelength, integrated and normalized to illustrate the percentage of the total collected at any one wavelength.

Figure 5: The modified fullerene PCBM, which functions as the donor in OPV devices. $^{35}$ 13

Figure 6: (A) P3HT semiconducting polymer. ${ }^{40}$ (B) PCPDTBT low energy semiconducting polymer. $^{41}$ (C) PEDOT:PSS polymer anode. ${ }^{42}$. 15

Figure 7: Energy diagram of a typical OPV device showing the driving forces behind electricity generation. ${ }^{17}$

Figure 8: J-V curve of an OPV device with and without illumination, showing the upward shift of the entire $\mathrm{J}-\mathrm{V}$ curve and subsequent strong effect of illumination on $\mathrm{J}_{\mathrm{SC}}$

Figure 9: Illustration of the fill factor calculation, which is the ratio of the maximum power divided by the theoretical maximum power. 23

Figure 10: Representative absorption spectra for PCPDTBT-P3HT:PCBM devices before and after annealing, showing annealing inducing a red-shift in the main 
amorphous peak ( 480 $\mathrm{nm}$ as-cast, and $\sim 510 \mathrm{~nm}$ after annealing) as well as crystaline shoulders at $\sim 540 \mathrm{~nm}$ and $\sim 600 \mathrm{~nm}$.

Figure 11: The first derivative of the simplified absorption spectra for a PCPDTBTP3HT:PCBM device as cast and annealed showing the existence of "shoulders" at 550 and $600 \mathrm{~nm}$ in the annealed spectra that are not present in the as-cast film............ 26

Figure 12: Schematic representation of the glass substrate with patterned ITO.............. 28 Figure 13: Schematic diagram of the ITO patterned glass substrate with a spin coated layer of PEDOT:PSS.

Figure 14: Schematic showing the remaining PEDOT:PSS layer, and the continuous scratches in red, both meant to electrical isolate the small ITO pad from the large ITO pad.

Figure 15: Schematic diagram showing the application of the active layer material on the patterned polymer anode and ITO.

Figure 16: Schematic of the remaining active material (orange) removed from the edges of the substrate.

Figure 17: Schematic diagram of a finished OPV device showing the approximate geometry and thickness of the various thin films.

Figure 18: A typical J-V curve focused on the power-producing region of the curve with the maximum power point in red, illustrating the origin of four electrical characteristics of a solar cell

Figure 19: Current and power plotted against voltage, illustrating the resolution of the approximation for maximum power. 
Figure 20: Electrical characterization jig, showing the eight pogo-pin connections

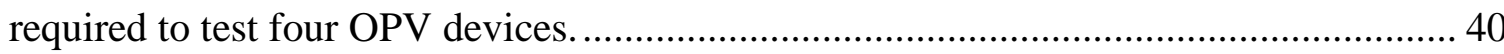

Figure 21: UV-Vis substrate holder inside of the nitrogen glove box environment........ 41

Figure 22: Schematic representation of the area of active layer sampled for optical density measurements.

Figure 23: Input light spectrum for the UV-Vis data collected showing low intensity on the edges of the spectrum.

Figure 24: Absorbance of the PEDOT:PSS layer and the substrate showing decreasing signal to noise ratio on the edges of the spectrum.

Figure 25: Standardized residuals versus the fitted values from the characteristic equation modeling the error found within the GLM model. 45 Figure 26: Standardized residuals plotted against the percent of data that have the associated residual value, visually representing the normal distribution of residuals from the model.

Figure 27: Dot-Plot of shunt resistance values showing small pixels, in red, with consistently lower shunt resistance, despite normalization to area. 50 Figure 28: Dot-Plot of short circuit current density showing relatively equal variance on the device level.

Figure 29: The main effects on the mean of short circuit current density, which show a large pixel variation with smaller pixels (b and c) performing more effectively, and a statistically significant effect from QD concentration much smaller than the observed pixel effects 53

Figure 30: Dot-Plot of open circuit voltage with one removed outlier in red. 53 
Figure 31: Interaction plot for the mean of open circuit voltage

Figure 32: Dot plot analysis for the fill factor response, showing the location of two outliers

Figure 33: Main effects on the mean of fill factor with the dominating factor of QD

concentration

Figure 34: Dot-plot of power conversion efficiency showing the removed outliers in red.

Figure 35: Main effects on efficiency showing small pixels outperforming large pixels and small loading of QDs having little negative effect on the efficiency.

Figure 36: Dot-plot of the response variable, series resistance, showing both outliers

in red.

Figure 37: Detail dot-plot of the response variable, series resistance, showing the

relative variance between treatments.

Figure 38: Main effects on the mean of series resistance.

Figure 39: Average normalized absorption spectra before devices are annealed. Each

line represents the average of six distinct spectra.

Figure 40: Average normalized absorption spectra after annealing at $110^{\circ} \mathrm{C}$ for ten

minutes.

Figure 41: Average normalized absorption spectra after annealing at $140{ }^{\circ} \mathrm{C}$ for ten

minutes.

Figure 42: Average normalized absorption spectra after annealing at $170{ }^{\circ} \mathrm{C}$ for ten

minutes. 
Figure 43: Average absorption spectra of devices without QDs showing a typical diffuse absorption before annealing and increasingly crystalline "shoulders" as anneal temperature increases. The color of the line denotes the value along the normalized absorption spectra.

Figure 44: Average absorption of devices containing 0.7 wt\% QDs before annealing and with each separate anneal treatment. The color of the line denotes the value along the normalized absorption spectra and the grey indicates off-scale absorption with the scale limited to the absorption of devices without QDs.

Figure 45: Average absorptivity spectra of devices containing $2.7 \mathrm{wt} \%$ QDs, showing a maximized absorption response at the $110^{\circ} \mathrm{C}$ anneal......................................... 70

Figure 46: Average absorption of devices containing $10.0 \mathrm{wt} \%$ QDs. 71

Figure 47: Main effects on the mean percentage difference between as-cast and annealed conditions, showing a large effect due to QDs with a smaller effect based on anneal temperature. 75

Figure 48: Main effects on the mean percentage difference between as-cast and annealed devices, showing a larger decrease in $\mathrm{V}_{\mathrm{OC}}$ due to QD loading than to anneal temperature.

Figure 49: Main effects on the mean percentage difference between annealed and ascast states' series resistance showing a smaller decrease in series resistance with QD loading and a nuetral response with anneal temperature.

Figure 50: On the left, a topography scan of a PCPDTBT-P3HT:PCBM OPV annealed at $110^{\circ} \mathrm{C}$ for ten minutes. On the right, a phase angle scan of the same position and surface. 79 
Figure 51: EFM phase angle scans of a PCPDTBT-P3HT:PCBM OPV annealed at $110^{\circ} \mathrm{C}$ for ten minutes: top showing normally distributed noise centered about $0.08 \mathrm{~V}$, bottom a $5 \mathrm{~V}$ bias clearly showing two distinctly different electric field responses with an intermediate response surrounding them. 80 Figure 52: Topography scan of the PCPDTBT-P3HT:PCBM control annealed at $110^{\circ} \mathrm{C}$ for ten minutes, showing an overall smooth film and several small particles and an RMS value of $0.60 \mathrm{~nm}$. 82 Figure 53: Topography scan of a PCPDTBT-P3HT:PCBM OPV sensitized with 0.25 $\mathrm{mg} / \mathrm{mL}$ QDs and annealed at $110^{\circ} \mathrm{C}$ for ten minutes, showing increased RMS roughness of $0.81 \mathrm{~nm}$ and similar particles on the surface

Figure 54: Topography scan of a PCPDTBT-P3HT:PCBM OPV sensitized with 1.0 $\mathrm{mg} / \mathrm{mL}$ QDs and annealed at $110^{\circ} \mathrm{C}$ for ten minutes, showing further increased RMS roughness of $1.41 \mathrm{~nm}$ and similar particles on the surface. 83 Figure 55: EFM phase image of the PCPDTBT-P3HT:PCBM control after annealing at $110^{\circ} \mathrm{C}$ for ten minutes which exhibits substantially different electric field strengths at sizes below $50 \mathrm{~nm}$ in diameter. 83 Figure 56: EFM phase images of OPVs sensitized with $0.25 \mathrm{mg} / \mathrm{mL}$ QDs (top) and $1.0 \mathrm{mg} / \mathrm{mL}$ (bottom) that exhibit increased phase potential differences and increased areas of electric field response when compare to the control. 


\section{INTRODUCTION}

Countries across the world have a need for energy. Since the advent of fire, the consumption of resources for fuel has become embedded in human society. Whether it is a wood-fired oven or a personal computer, we use energy to accomplish our personal and societal goals. The production rate of energy was so vital to the industrialization of nations; sociologists correlated that to a measure of a nation's level of industrialization. ${ }^{1}$ In 2010 the world consumed 510.5 quadrillion British thermal units (Btu), which is 74.4 million Btu consumed per person. ${ }^{2}$ In more familiar terms, that is 21.8 megawatt hours consumed per person on the planet in 2010. Energy use is predicted to rise, especially in countries outside of the Organization for Economic Co-operation and Development (OECD). ${ }^{3}$ OECD member countries include economic powerhouses such as, the United States of America, Canada, Japan, and the majority of Europe. ${ }^{4}$ The consumption of energy in developed economies generally has lower growth rates than developing economies. ${ }^{5}$ When only the production of electricity is considered, the trend is accentuated. ${ }^{6}$ Electricity is the fastest growing delivered energy method and the electrification of areas that historically do not have access to the resource, which equates to 1.3 billion people, is the main avenue for its growth. ${ }^{6}$

There are many methods of electricity production around the world. The most common production methods, in order from largest to smallest growth rate, are coal, natural gas, renewables, nuclear, and liquids. ${ }^{6}$ Renewable electricity production is predicted to be the fastest growing source between 2010 and 2040, mainly due to increased production from hydroelectric generation and wind generation; however, most 
renewable energy production methods carry with them large immediate costs. The benefits of renewable energy production extend beyond economics, because renewable energy production frees resources associated with securing that energy from another country through trade or force. ${ }^{6}$ Further, since most electricity production occurs in large, centralized installations, like dams for hydroelectric power and wind farms for wind energy, the generated electricity must be transported to its end-user. ${ }^{7}$ That transportation requires huge infrastructure and that infrastructure has associated power loss. Solar energy, as a decentralized electricity generation source, does not require pre-existing infrastructure to deliver power to the end-user. This means that solar has added benefits in locations with poor existing infrastructure or no infrastructure, and compatibility in applications that require stand-alone operation.

If we put aside the niche benefits of solar electricity generation, the most straightforward method of comparison between energy sources is the economic metric Levelized Energy Cost (LEC). Put simply, the LEC of an energy source is the cost of an energy production system divided by the amount of energy that system can produce during its useful life. ${ }^{8}$ This measure standardizes the costs of an energy production method per unit of energy. Conventional coal power plants entering service in 2019 have an estimated LEC of $95 \$ / \mathrm{MWh}$, with solar photovoltaics at $130 \$ / \mathrm{MWh} .{ }^{9}$ In the case of solar energy, the majority of the cost to the customer is in the initial investment, with almost no maintenance costs and no fuel costs. Thus, the LEC of photovoltaic systems can improve by decreasing the initial cost of the solar cell, extending the lifetime of the unit, or by increasing the amount of energy the unit can produce. The initial cost of silicon solar cells is fixed mainly by the cost of the highly purified, energy intensive 
material, electronic grade silicon. ${ }^{10}$ Since silicon is an indirect band-gap material, a thick film, around $400 \mu \mathrm{m}$, of material is required for good light absorption. ${ }^{11}$ Further, silicon solar cells are limited by thermodynamics to fall under the Shockley-Queisser limit, which places the maximum efficiency around 30\%. ${ }^{12}$ There are methods of exceeding the limit; however, the flaws in silicon as a material, low-band gap, indirect band gap, high dielectric constant, and consequently high reflectivity all pose great technical challenges. Simply put, silicon solar cells are unlikely to produce a favorable LEC since the only avenue for substantial improvement in LEC is in lowering the initial capital cost contribution to LEC.

If silicon solar cells are unlikely to produce a favorable LEC, and decentralized site-specific electric power generation is needed, how will this need be filled? One alternative is the polymer solar cell. Polymer solar cells are produced by dissolving a semiconducting polymer and depositing the dissolved polymer on a substrate between two dissimilar electrode materials. The ability to be dissolved, deposited in a wet form, and dried, all the while retaining semiconducting electrical properties, means that polymer solar cells can be produced on a roll-to-roll basis, like newspapers or magazines, allowing high throughput to lower production costs and consequently the LEC. ${ }^{13}$ Polymer solar cells also rely on much thinner films, around $100 \mathrm{~nm}$, meaning that material costs are much lower than the thick silicon based solar cells. ${ }^{14}$ The deposition of the film is a room-temperature process meaning that, unlike silicon, there is very little energy required to create the active layer. Polymer solar cells are lightweight, durable, cheap, and biodegradable making them a perfect fit for decentralized power production in nonOECD countries. ${ }^{15}$ Unfortunately, polymer solar cells are not perfect from a solar cell 
design standpoint. The active layer material lacks a high dielectric constant $(\varepsilon \approx 3.4) .{ }^{16}$ This low dielectric constant allows organic photovoltaics (OPVs) to absorb more light at higher incident angles throughout the day; however, a low dielectric constant fails to shield the columbic attraction between the excited electron and hole. Those excited electrons are still coulombically bound to a hole and must diffuse to a junction between donor and acceptor materials to separate, introducing complications in charge transfer states within the cell. ${ }^{17}$ The excitonic nature of OPV devices requires incredibly high interfacial surface area which is generally achieved by creating an intimate mixture of donor and acceptor materials, known as a bulk heterojunction. The material with the best spontaneous heterojunction formation, $\mathrm{P} 3 \mathrm{HT}$, is not well matched to the solar spectrum because of its relatively wide band-gap of $1.9 \mathrm{eV} .{ }^{18}$ The biodegradability of the devices stems from oxygen and water assisted photo-corrosion of the donor materials leading to shorter device lifetimes. Large scale OPVs have shown device lifetimes of 5000 hours in accelerated lifetime testing. ${ }^{19}$ Non-accelerated lifetime experiments conducted by Konarka Technologies Inc. show no degradation in over a year of rooftop testing. ${ }^{20 E r r o r !}$ eference source not found. Given the detriments of polymer solar cells, the best ways of improving device LEC are to improve the lifetime of the device or to improve the power conversion efficiency of the device, which assumes that the technologies will remain rollto-roll compatible for lower costs through increased production. A method of increasing the power conversion efficiency involves changing how a particular OPV responds to the light spectrum. This sensitization can be accomplished through adding synthetic nanocrystals known as quantum dots. ${ }^{21}$ Quantum dots have several advantages when considered for this application. Quantum dots have tunable optical and electrical 
properties, meaning that they can be applied in a wide variety of material systems. ${ }^{22}$ Second, since the quantum dots are semiconductor nanocrystals, they could increase absorption simply through scattering effects. This paper examines attempting to improve performance in polymer solar cells through increased absorption using fluorescent nanocrystals known as quantum dots. 


\section{BACKGROUND}

The goal of any solar cell is to convert the energy coming from the sun into usable electrical energy. This seems a simple task on the surface; however, the sun's energy does not arrive neatly packaged in multiples of one particularly sized "box" of energy, like the burst of energy from a piston in an engine. Since the sun is so massively energetic, its energy arrives in a multitude of wavelengths, all at different intensities related to the surface temperature of the star, which is known as a black body radiator. ${ }^{23}$ After the sun generates the spectrum of light, it travels through space in all directions and a small portion travels directly toward earth. Since there are relatively few obstructions present in the ultra-high vacuum of space, the light spectrum from the sun arrives generally intact and begins traveling through the earth's atmosphere. The earth's atmosphere is full of gaseous molecules that can absorb light or scatter it, making the spectrum reaching the earth's surface, the spectrum useful for terrestrial solar cell applications, a function of concentration of these attenuation mechanisms and the path length of atmosphere traversed. ${ }^{24}$ This real world condition makes comparison of solar cell performance overly difficult, if not nearly impossible. Without direct comparison, scholarly research of solar cells would be without focus or direction and reported industrial achievements could be manipulated. The combination of these needs and the emergence of solar energy as a resource prompted the American Society for Testing and Materials (ASTM) to accurately record the solar spectrum and publish a standard reference for testing and comparing photovoltaics (Figure 1). ${ }^{25}$ 


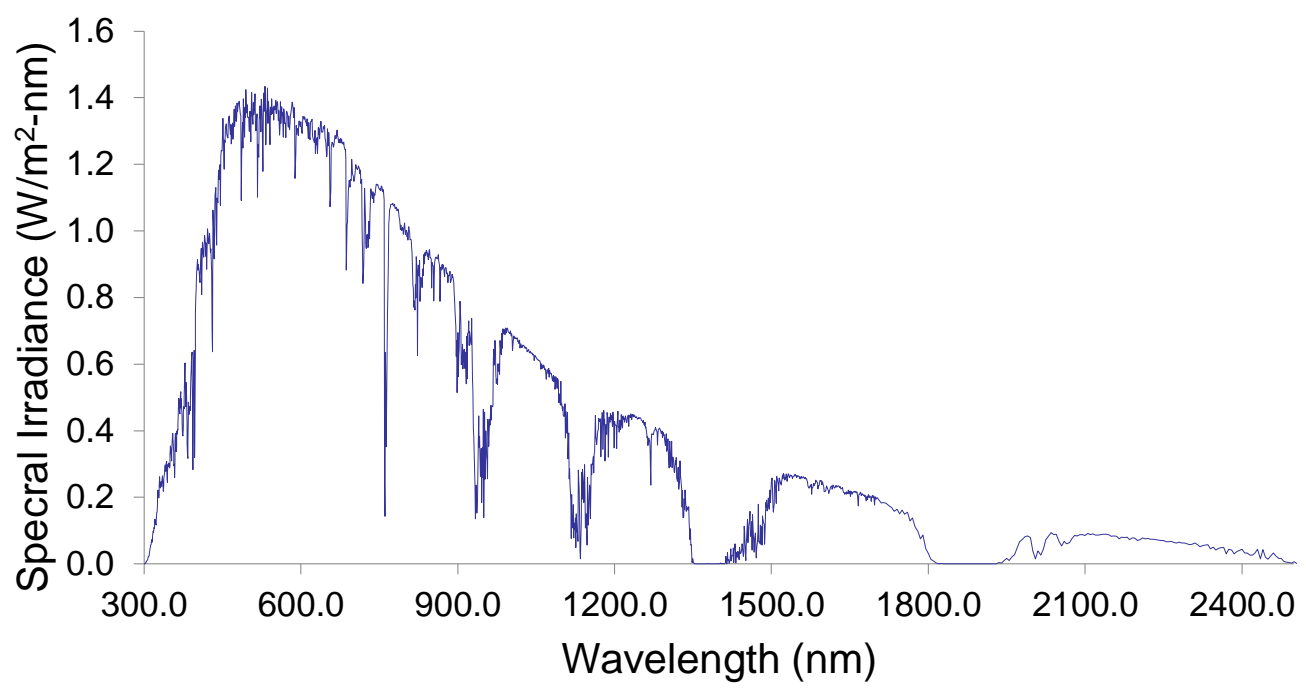

Figure 1: ASTM G173 standard solar spectral emission at $1000 \mathrm{~W} /\left(\mathrm{m}^{2} \mathrm{~nm}\right)$.

The downward spikes, or decreases in transmission, are linked to absorption and attenuation mechanisms from the earth's atmosphere. Each degree of tilt away from the zenith increases the path length, and subsequently the effect of atmospheric attenuation. As such, the ASTM standard includes the path length through the atmosphere in units of "air-masses" (AM). AM is defined by,

$$
A M=\frac{1}{\cos \theta}
$$

where $\theta$ is the angle from zenith, perpendicular to the earth's surface. For instance, the spectrum derived from the zenith, when light comes directly perpendicular to the earth's surface is referred to as AM 1.0 because it passes through exactly one thickness of the earth's atmosphere. AM 1.5, the most widely used standard, passes through the atmosphere at an angle of $48.2^{\circ}$ from zenith while AM 2.0 comes in at $60.1^{\circ}$ (Figure 2). 


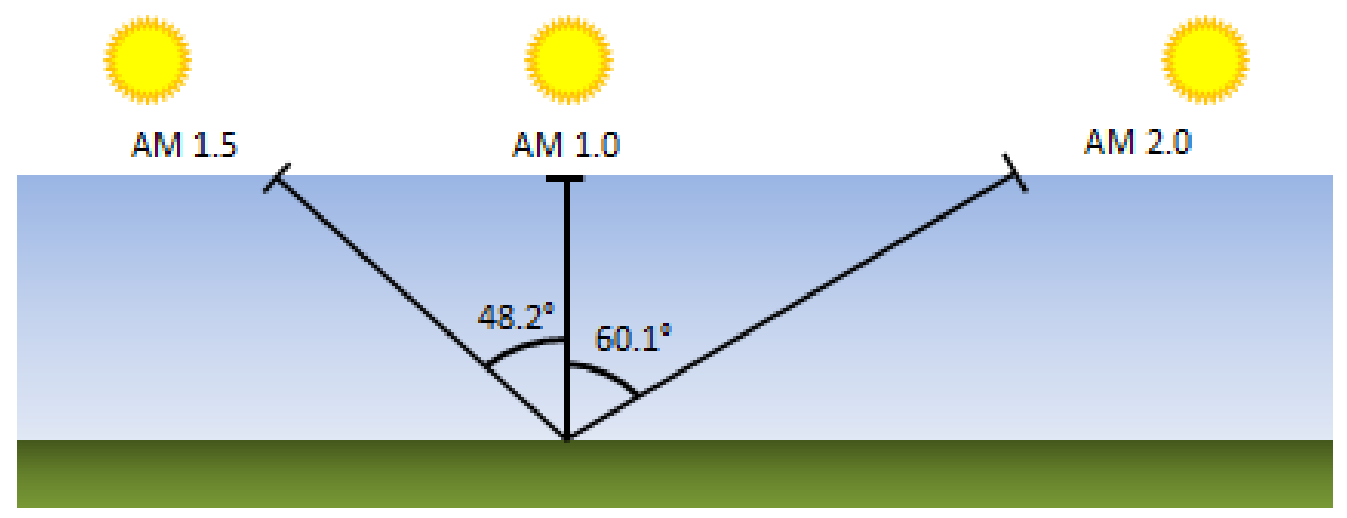

Figure 2: Schematic representation of the concept behind the air mass measurement, showing the tilt away from zenith required for AM 1.5 and AM 2.0.

Now that the spectrum of light has a standard intensity per wavelength, artificial solar simulation can be scaled and corrected so the strengths of the solar cell system are represented than false data from poor testing procedures, and scholarly calculations can be based in an agreed model of reality.

When designing a solar cell, a complication arises from the nature of light and light absorption. Each wavelength in the spectrum is representative of a discrete packet of energy known as a photon. The amount of energy contained in a photon is inversely proportional to the wavelength it travels and is given by:

$$
E=\frac{h c}{\lambda} \cong \frac{1240 \mathrm{eV} \mathrm{nm}}{\lambda}
$$

where $h$ is Planck's constant, $c$ is the speed of light, and $\lambda$ is the wavelength of light. ${ }^{26}$ Even though a $300 \mathrm{~nm}$ wavelength photon is twice as energetic as a $600 \mathrm{~nm}$ wavelength photon, their relative contribution to power generation within the solar cell is nearly identical. To explain why this happens, we need a more detailed picture of how photons create electricity in a solar cell. The absorption of photons drives the excitation of a solar 
cell by forcing electrons to jump to their next highest allowable energy state. If the incoming photon contains enough energy to excite the electron, and the conditions of the photon-electron interaction are favorable, the electron absorbs the energy of the photon; however, the difficulty of the jump to the next allowable energy, known as the band gap, is a constant material property. The band gap represents the maximum energy a solar cell can extract from a photon. From an absorption perspective, this allows for three sets of photon-electron interaction: the photon contains exactly the same energy as the band gap of the material; the photon contains more energy than the band gap of the material; lastly, the photon contains less energy than the band gap of the material. In the later situation, the photon is simply not absorbed which generally leads to transmission. The cases where the electron is at least, or more, energetic as the band gap, the photon can be absorbed. For instance, the glass in a window has a large band gap energy, so large in fact, the visible spectrum cannot excite the electrons in the glass while photons in the ultraviolet (UV) are absorbed. This "turn-on" in absorption is called band edge absorption. ${ }^{27}$ The electron that absorbed more energy than was required to jump the band gap goes through a process called thermalization in which it loses energy to heat as it relaxes to an allowed state within the conduction band. ${ }^{27}$ This thermal energy does not count toward the goal of converting sunlight into electricity, and once thermalization has completed, the two electrons end up in the same excitation state. Therefore, we must consider the band gap that sets the amount of energy that can be absorbed, as well as the number of photons that contribute a band-gap-sized portion of their energy to electricity generation in the solar cell. Thus, it becomes useful to think of the solar spectrum in terms of photon flux per 
wavelength, the number of photons of any particular wavelength striking the earth's surface per unit time, instead of power density (Figure 3).

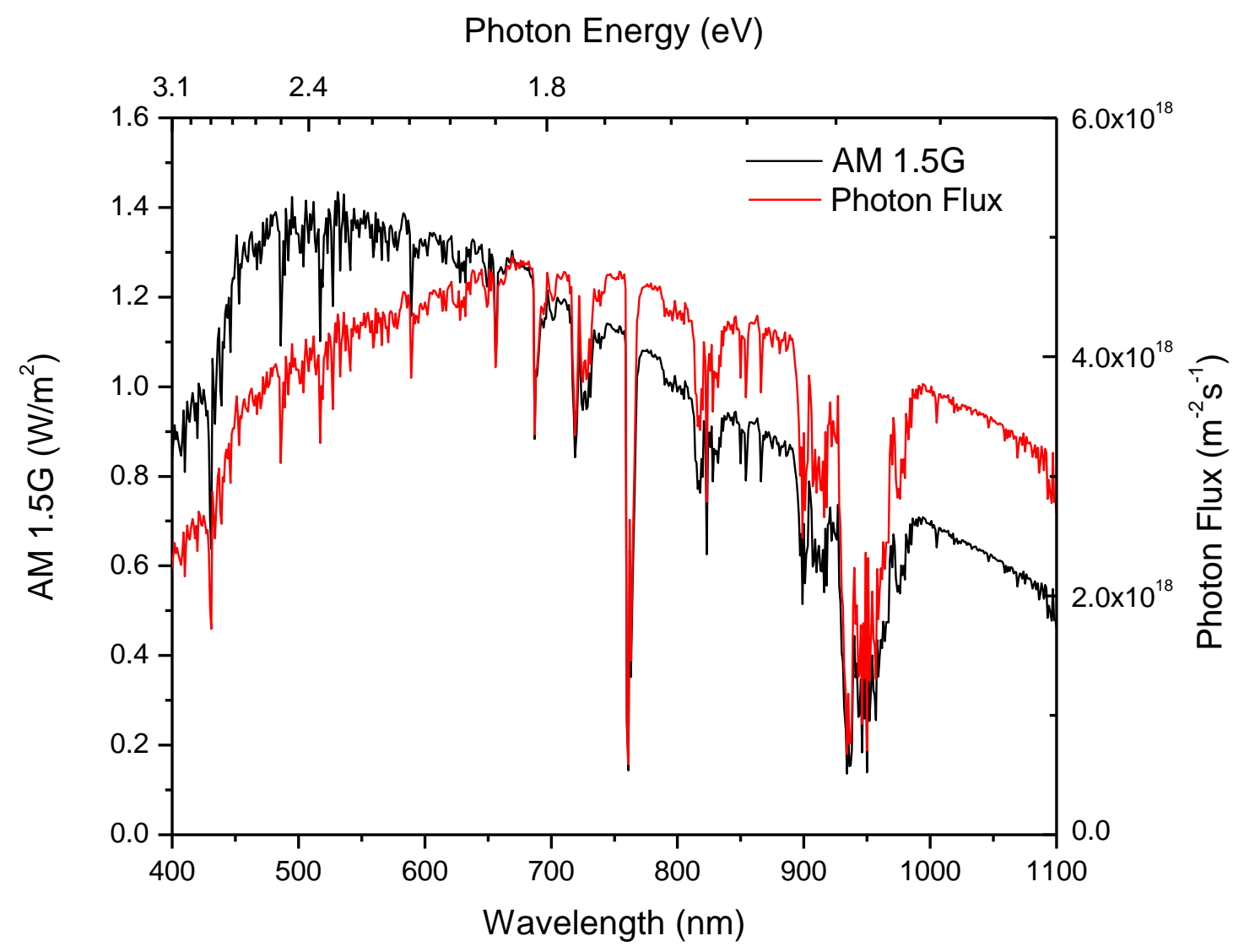

Figure 3: Focused view of AM 1.5G expressed as energy per wavelength (black) and photon flux (red). The standard AM $1.5 \mathrm{G}$ spectrum extends from $280 \mathrm{~nm}$ to $4000 \mathrm{~nm}$.

The difference between the two perspectives is obvious; however, photon flux versus photon intensity is far from helping to inform a design decision concerning the best band gap for power generation. Finding the percentage of photons harvested below a specific wavelength and the percentage of the total power harvest below a specific wavelength offers a direct, decision-making comparison between the two perspectives. In order to obtain this graph we must integrate the terrestrial solar spectrum and the solar photocurrent spectrum, the photon flux per unit time and area, with respect to wavelength. Then to compare the two spectra we must normalize and multiply by 100 to 
obtain percentages. The resulting graph informs the reader to what percentage of power could be harvested if thermalization did not exist, the percentage of solar photocurrent a given band edge harvests (Figure 4).

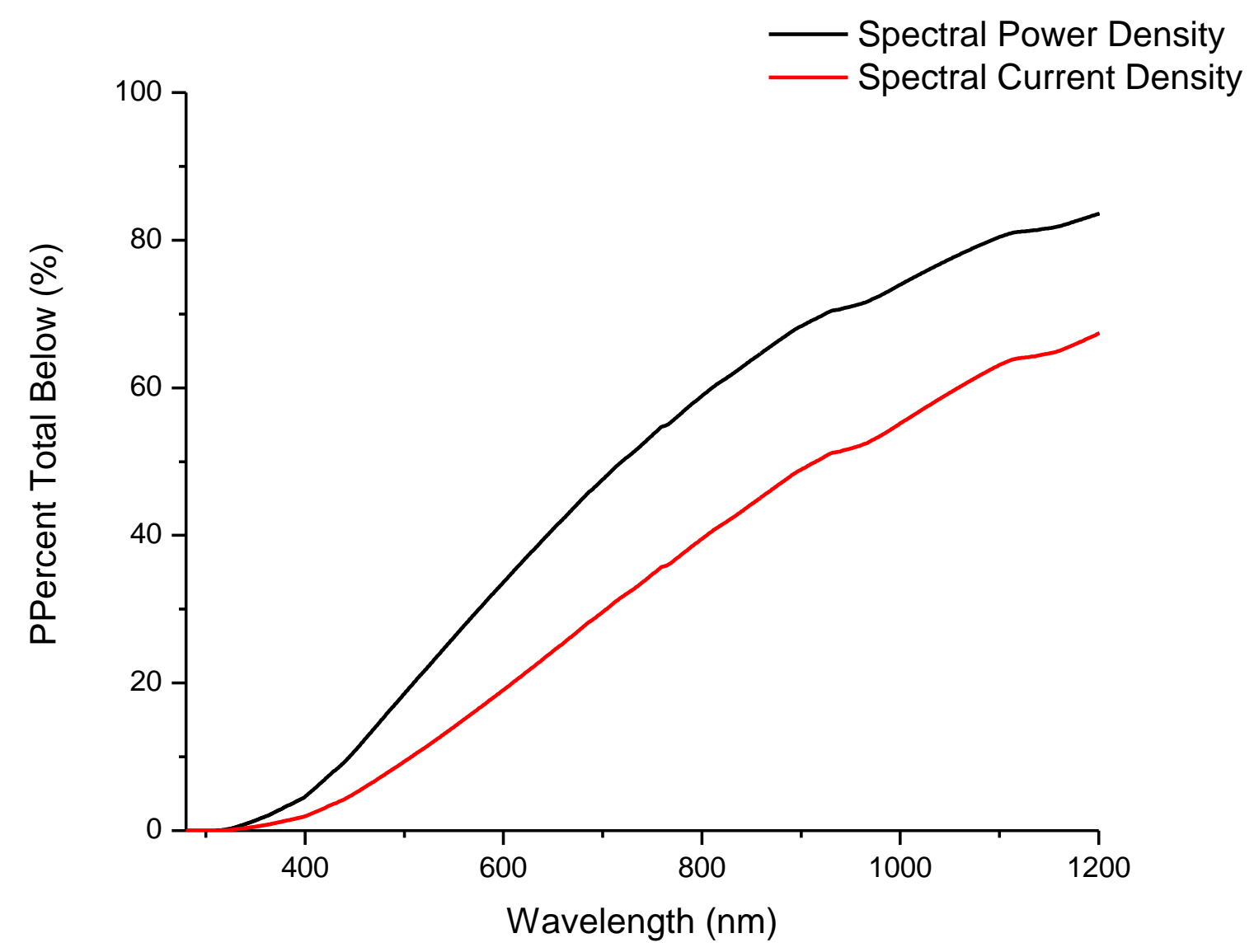

Figure 4: Spectral power density, the amount of power collected at any one wavelength, and spectral current density, the number of photons falling at any one wavelength, integrated and normalized to illustrate the percentage of the total collected at any one wavelength.

For instance, if we wanted to collect $60 \%$ of the total power emitted by the sun, we would collect every wavelength at or below $\sim 800 \mathrm{~nm}$. If we wanted to collect $60 \%$ of the total photons, we would collect every wavelength at or below $\sim 1050 \mathrm{~nm}$.

A final complication arises from the fact that simply because a material can absorb a photon does not mean that the material will absorb the photon. The ratio of 
photons incident to the surface of a material, photons that could be absorbed, to the free charge carriers generated by the solar cell, the photons that were absorbed, is known as the external quantum efficiency (EQE). Band edge absorption says that the EQE of a material is a step function that abruptly changes at the material's band gap energy; however, real world materials exhibit a gradual increase in EQE approaching the band gap energy from shorter wavelengths with a steep decline afterwards. This is because in the real world, materials are flawed and photon-electron interaction conditions are not always favorable. The practical consequences of this means that the actual energy captured by the solar cell will always be less than the number of photons beneath a certain band edge multiplied by the energy of the band gap.

\section{Semiconducting Polymers and the Bulk Heterojunction}

Despite their complex nature, polymer electronics are widespread, both commercially and in a laboratory setting. ${ }^{28}$ Polymer based light emitting diodes have been utilized in lighting and display technology. ${ }^{29}$ Basic electronics components like resistors, capacitors, and transistors have been created with combinations of conductive and semiconductive polymer elements. ${ }^{30}$ It comes as no surprise that Alan J. Heeger, Alan MacDiarmid, and Hideki Shirakawa won a Nobel Prize in 2000 for their work demonstrating macroscopic electronic processes in polymers. ${ }^{31}$

Semiconductive polymers enabled the creation of the polymer solar cell. ${ }^{32}$ Semiconducting polymers conduct charge through their molecular backbone consisting of alternating single bonded, known as $\sigma$-bonded, and double bonded, known as $\pi$-bonded, carbon-carbon bonds. ${ }^{33}$ This alternation between $\sigma$ and $\pi$ bonding is known as $\pi$ conjugation and allows charge to delocalize along the polymer chain. ${ }^{34}$ Charge can also 
transfer between polymer chains, but this process is more difficult. The bulk properties of an organic material are highly dependent on the order and closeness of each polymer backbone to one another.

Conductive polymers do not create solar cells on their own. Polymer solar cells are created using one or more semiconducting polymers that serve as electron donors. In this manner, donor polymers function as an n-type material, and hole-conductor. It is important to realize that because of the nature of charge transport in $\pi$-conjugated polymers, the polymer contributes nearly all of the hole mobility without contributing significantly to electron mobility. The material that functions as the acceptor, p-type material, and electron conductor is [6,6]-Phenyl C61 butyric acid methyl ester (PCBM), which is a fullerene modified for solubility in non-polar solvents (Figure 5).

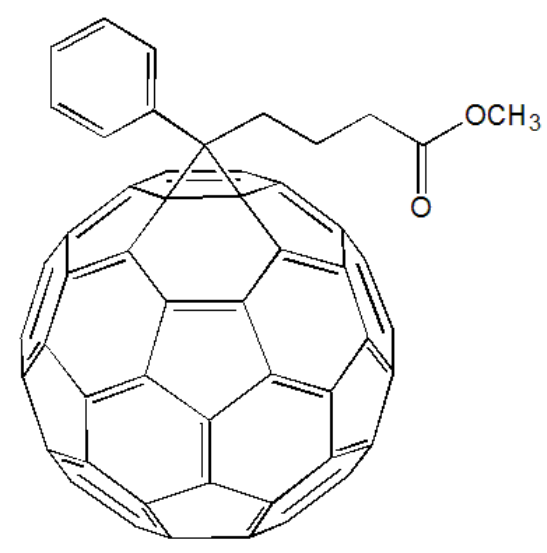

Figure 5: The modified fullerene PCBM, which functions as the donor in OPV devices. ${ }^{35}$ Despite extensive research into alternative acceptor materials, PCBM has remained the most widely used acceptor material because of its high electron affinity, charge carrying characteristics, and device morphology within a bulk heterojunction device architecture. ${ }^{36}$

To create an OPV device, donor and acceptor layers must be deposited between two electrodes. Initial device architectures used a "sandwich" method, depositing the 
anode, the donor material, the acceptor material, and the cathode. Devices using this architecture exhibited power conversion efficiencies close to $1 \% .^{37}$ This poor performance is due to the relatively small heterojunction area, the area where acceptor material and donor material touch. When P3HT (acceptor), PCPDTBT (acceptor), and PCBM (donor) are mixed, they form an intimate mixture with distinct phases on the nanometer scale, which is known as a bulk heterojunction $(\mathrm{BHJ}){ }^{38}$ This spontaneous process is essential to creating high efficiency OPVs as the donor-acceptor interfacial surface area drives the creation of free charge carriers within the OPV active layer. Since excitons, excited electron-hole pairs, have a fixed diffusion distance driven by the recombination rate, the morphology of the $\mathrm{BHJ}$ must be strictly controlled. If the regions within the $\mathrm{BHJ}$ are too large, the material is underutilized which drives down efficiency. If those regions are too small, free charge carriers must navigate a torturous pathway to the electrodes, which drives down efficiency by encouraging recombination of free charge carriers with part of a different electron-hole pair. Annealed P3HT:PCBM forms BHJ regions approximately 10-20 nm wide, which just happens to be the diffusion length of an exciton within the material. ${ }^{39}$ It is for this reason that, despite P3HT being poorly matched to the solar spectrum, P3HT-PCBM devices serve as the benchmark against new device designs.

The polymers investigated in this paper, which for clarity create one BHJ device design, are (Figure 6):

A. Regioregular Poly(3-hexylthiophene-2,5-diyl) (P3HT), which drives the morphology and absorption characteristics of the solar cell; 
B. Poly[2,6-(4,4-bis-(2-ethylhexyl)-4H-cyclopenta [2,1-b;3,4-

b']dithiophene)-alt-4,7(2,1,3-benzothiadiazole)] (PCPDTBT), which

serves as a narrow band-gap polymer working in conjunction with P3HT

to utilize a greater portion of the solar spectrum;

C. and Poly(3,4-ethylenedioxythiophene) Polystyrene sulfonate

(PEDOT:PSS), which serves as a transparent polymer anode, buffering layer, and electron blocking layer.

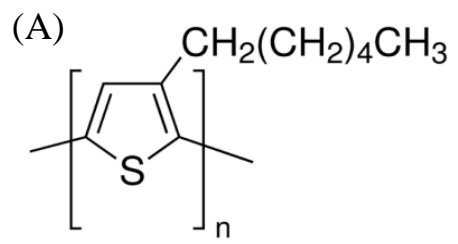

(C)

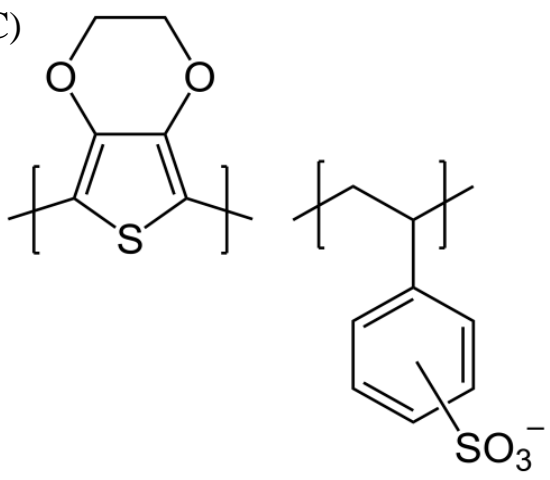

(B)

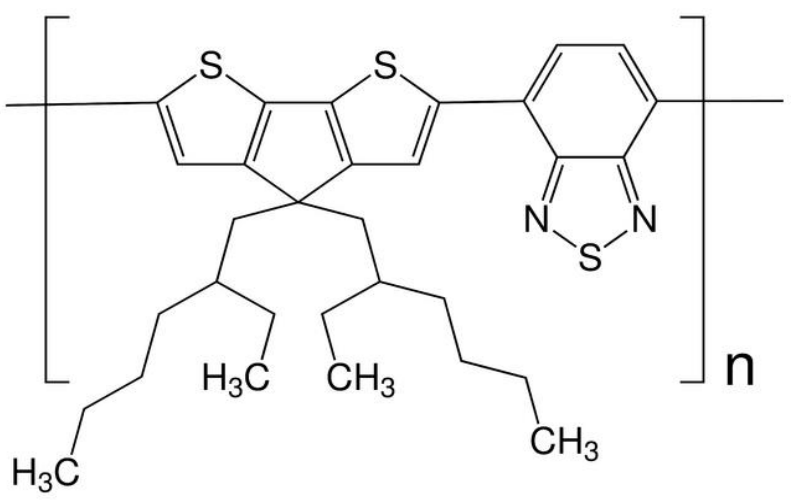

Figure 6: (A) P3HT semiconducting polymer. ${ }^{40}$ (B) PCPDTBT low energy semiconducting polymer. ${ }^{41}$ (C) PEDOT:PSS polymer anode. ${ }^{42}$

As said earlier, P3HT is poorly matched to the solar spectrum. With a band gap of $1.9 \mathrm{eV}$, the band edge absorption ends around $650 \mathrm{~nm}$, which corresponds to a little more than $20 \%$ of the available photons in the solar spectrum. PCPDTBT, the other electron donor material, has a band gap of about $1.4 \mathrm{eV}$ meaning that PCPDTBT can absorb photons out to $\sim 880 \mathrm{~nm}$, which corresponds to just under half of the available photons. 
PCPDTBT:PCBM BHJ devices exhibit poor charge carrier mobility and thus reduced efficiencies. ${ }^{43}$ The purpose of mixing the two acceptor materials becomes twofold: the relatively narrow band gap of the PCPDTBT increases the amount of the spectrum that can be absorbed, while the robust morphology and charge carrying capability of the P3HT mitigates a decrease in device performance.

\section{Creating Free Charge Carriers}

Since semi-conducting polymers transport charge differently than the metals and silicon based semiconductors commonly found in our electronics, they cannot be described using band theory. Those semiconductors rely on free charge carriers to transport charge through the material, while all the electrons participating in the bonding of the solid are predominately "locked." Semiconducting polymers, like P3HT and PCPDTBT, can only transport charge with electrons participating in the molecular bond. Since only the bonded electrons participate, semiconducting polymers are described using their highest occupied molecular orbital (HOMO), and their lowest unoccupied molecular orbital (LUMO), which are analogous to the valence and conduction bands respectively. As such, the LUMO and HOMO levels of the materials participating in photon absorption and exciton creation provide a forum to describe the underlying physics of OPVs. The difference between the HOMO of the donor material and the LUMO of the acceptor material sets a maximum limit to the open circuit voltage, while the LUMO-LUMO offset and the difference in work functions of the electrode materials have practical device level consequences on the $\mathrm{V}_{\mathrm{OC}}{ }^{44}$ In this case there are two donor materials, $\mathrm{P} 3 \mathrm{HT}$ and PCPDTBT, which both participate in exciton generation, which complicates any simplistic explanation of observed phenomena. Since the absorption of a photon results in the generation of an exciton, and the low dielectric constant of the 
organic materials do not shield the columbic attraction between the electron and hole, the exciton remains bound until it reaches an interface between donor and acceptor material. Once an interface is reached the LUMO-LUMO offset between the donor material and the acceptor material drives the dissociation of the excitonic state. ${ }^{45}$ Experimental observations have shown a LUMO-LUMO offset of $0.3 \mathrm{eV}$ sufficient separate the carriers; however, the P3HT-PCBM system exceeds this number with an offset of $1.1 \mathrm{eV}$. ${ }^{46}$ In an energy diagram, the electrode materials may be plotted using their work function, the minimum energy required to remove an electron from a material, with the polymer anode, polymer donor HOMO and LUMO level, and fullerene acceptor HOMO and LUMO level (Figure 7).

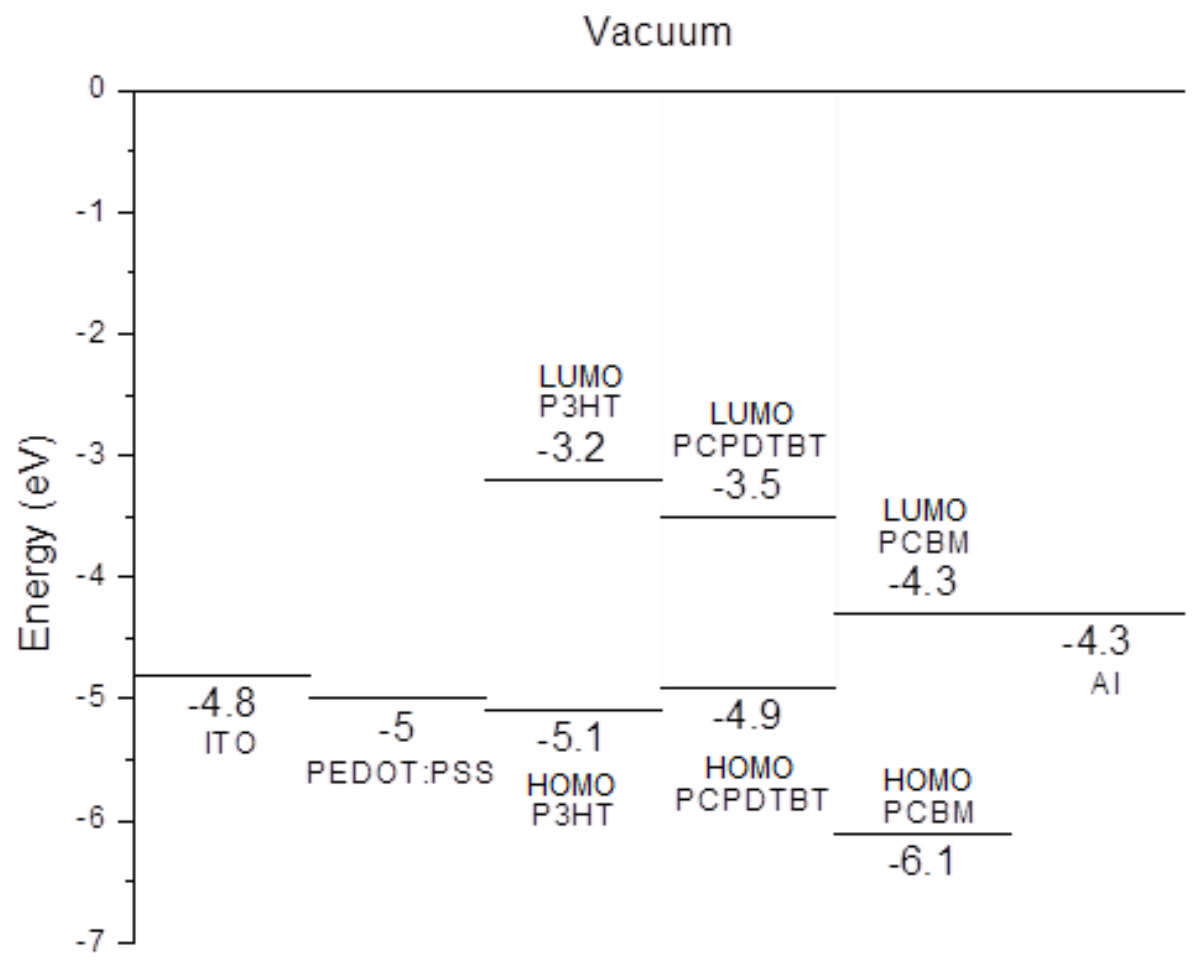

Figure 7: Energy diagram of a typical OPV device showing the driving forces behind electricity generation. ${ }^{17}$ 
When two different conductors are in equilibrium, there is a built in electric potential set by the difference between the two materials' work functions. This potential breaks the inherent symmetrical condition in BHJ devices, and drives the free charge carriers to the electrodes and allows the OPV to function. Since electric potential is distant dependent, the BHJ film thickness, which drives absorption, must be balanced with the built in electric field, which drives charge extraction.

\section{Quantum Dots}

Quantum dots (QDs) are semiconductor nanocrystals that fluoresce when exposed to high-energy wavelengths of light. ${ }^{47}$ QDs differ from organic dyes because their fluorescence peak can be tuned by varying their size, a property known as quantum confinement. ${ }^{48}$ This quantum mechanical effect arises when the excited electron-hole pair radius, the Bohr Exciton radius, is larger than the material conducting the electron, generally less than $10 \mathrm{~nm}$ wide. The effect increases the band-gap of the semiconducting material from the value of the bulk material toward the value of discrete atomic states.

Taken out of a theoretical context, not all QDs are created equally. The quality of the QD crystal has a huge effect on its performance. In fact, synthesized QDs have an associated quantum efficiency describing how many photons absorbed are converted to photons emitted. ${ }^{49}$ Things like surface defects, multiple ligand layers, and elevated operating temperatures can drive down the quantum efficiency of a QD. Multiple ligand layers and the operating temperature can generally be precisely controlled; however, since the crystals are so small and contain so few atoms, surface defects in QDs are likely. Surface defects act as electron trapping regions. In order to overcome this issue, QDs can be fabricated with a "shell" around the photoactive core made of a wider band 
gap material. ${ }^{50}$ This is called a type 1 core shell QD. ${ }^{51}$ The QDs used in this study have CdSe cores with a $\mathrm{ZnS}$ shell. The $\mathrm{ZnS}$ has a wider band gap than the CdSe, meaning that the $\mathrm{ZnS}$ can transmit light while the CdSe absorbs light and that the excited electron now has an additional barrier to pass in order to escape radiative recombination. Thus the size of the core is still the controlling factor for tuning the emission peak, and the quantum efficiency is bolstered by the added surface passivation and additional barrier to nonradiative decay of excited electrons.

QDs can be stabilized to reduce the likelihood agglomeration by attaching a polymeric ligand to the surface of the $\mathrm{QD}$, which has the added benefit of allowing QDs to be colloidaly suspended in non-polar solvents. The ligand, while increasing the total volume of the QD, passivates the shell surface and ideally does not contribute substantially to decreasing the quantum efficiency or red shifting the fluorescence peak. ${ }^{52}$

\section{Solar Cell Characterization}

Solar cells, much like other diodes, are characterized through the creation of a current-voltage curve (J-V curve), which is simply the current passed through the device at any one voltage. A resistor, having no diode characteristics, exhibits a linear J-V curve. This means that the current increases with the voltage at the same rate over all voltages and the inverse of the slope of that line gives the resistance value. J-V curves belonging to diodes are slightly more complicated in that the rate at which the current increases, or decreases, with voltage is not constant. This non-linearity gives rise to the electronic devices of the modern age. Producing power further complicates the J-V curve. Solar cells exhibit $\mathrm{J}-\mathrm{V}$ curves that are dependent on their degree of illumination, which means that they can produces power from sunlight. Illumination of a solar cell shifts the $\mathrm{J}-\mathrm{V}$ 
upwards because absorbed light creates current when the diode would otherwise not produce current (Figure 8).

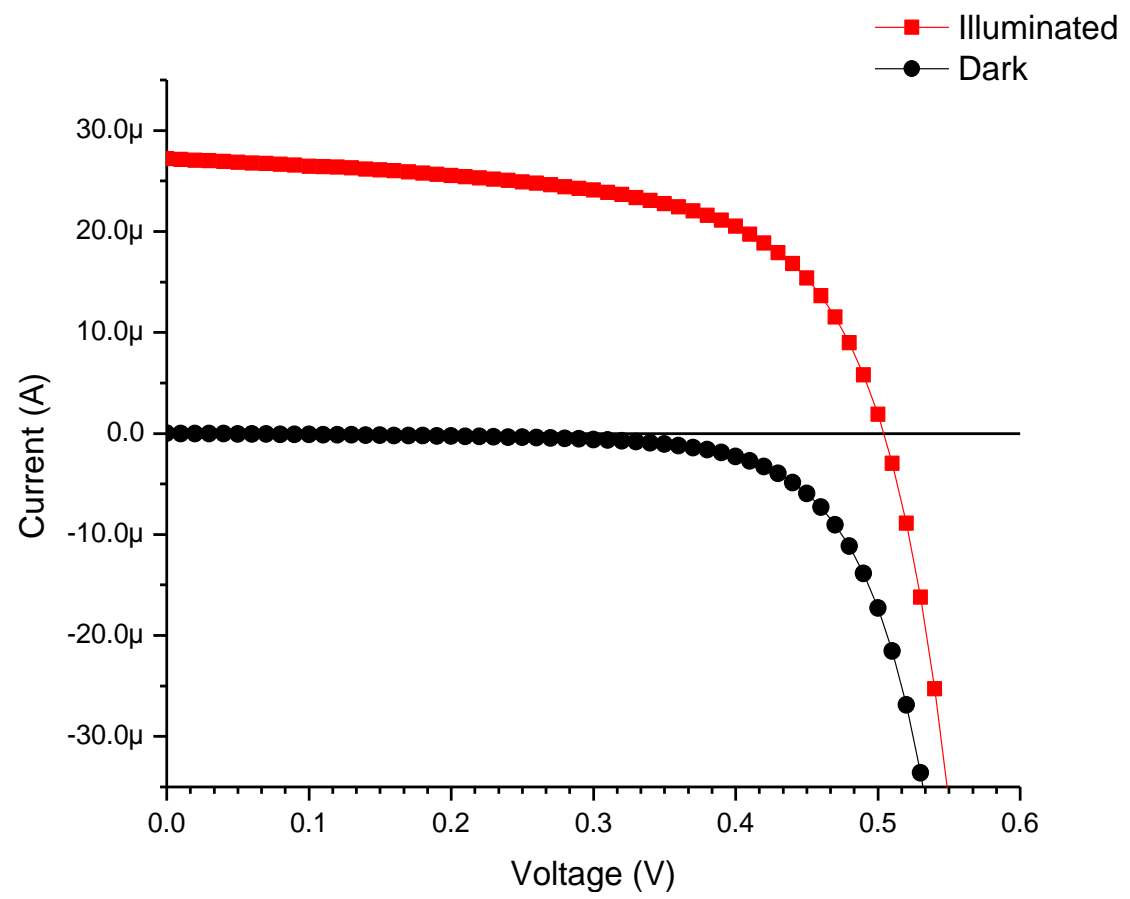

Figure 8: J-V curve of an OPV device with and without illumination, showing the upward shift of the entire $\mathbf{J}-\mathrm{V}$ curve and subsequent strong effect of illumination on $\mathbf{J}_{\mathrm{SC}}$.

If the amount of light is quantified, the temperature held constant, and the distance between voltage points short enough, an incredibly detailed electrical characterization is possible. Like any characterization, each characteristic value infers a distinct condition within the individual device; however, unlike inorganic solar cells, the underlying physics responsible for each response is often not straightforward and generally driven by multiple competing factors.

The basic electrical characteristics of any solar cell are the open circuit voltage $\left(V_{\mathrm{OC}}\right)$, the short circuit current density $\left(\mathrm{J}_{\mathrm{SC}}\right)$, series resistance $\left(\mathrm{R}_{\text {Series }}\right)$, shunt resistance $\left(\mathrm{R}_{\text {Shunt }}\right)$, the maximum power $\left(\mathrm{P}_{\mathrm{Max}}\right)$, the power conversion efficiency (PCE), and the fill 
factor $(\mathrm{FF}){ }^{53}$ These basic measures of performance serve as a fundamental comparison between all solar cells.

Open circuit voltage is strictly defined as the chemical potential between the cathode and anode of the solar cell. In silicon solar cells, it represents the contact potential of the p-n junction created by Fermi-level equalization. ${ }^{54}$ In polymer solar cells it is limited by, and linearly correlated to, the difference between the HOMO level of the donor and the LUMO level of the acceptor. Unlike their silicon counterparts, the LUMO level of the acceptor can decrease with crystallinity, decreasing the open circuit voltage. ${ }^{55}$ While the LUMO-HOMO offset limits the $\mathrm{V}_{\mathrm{OC}}$, the effectiveness of the exciton scission process can drive down observed $\mathrm{V}_{\mathrm{OC}}$ through accumulated charges in the LUMO level of the acceptor and HOMO level of the donor. The LUMO-LUMO offset of the donor and acceptor materials is mostly responsible for exciton scission; however, the built in potential created by the work functions difference between anode and cathode drive ambipolar diffusion ${ }^{56}$ For instance, an OPV with a large LUMO-HOMO offset and LUMO-LUMO offset created with electrodes of similar work functions would not function efficiently since the solar cell is electrically symmetrical and generated charge carriers would not be driven to flow out of the device. An OPV with a small LUMOHOMO offset, large LUMO-LUMO offset, and a large difference in electrode work function would be very efficient at exciton scission and free charge carrier collection, but the resulting potential of the collected charge would be small, giving a lower $\mathrm{V}_{\mathrm{OC}}$. Although the $\mathrm{V}_{\mathrm{OC}}$ is often considered simply a consequence of the materials used to construct the device and their resulting morphology, the complex nature of the materials and their morphologies create increasingly complex consequences. 
Short circuit current is the current passing through the solar cell when the voltage difference between the cathode and the anode is zero. In more fundamental terms, the short circuit current is given by:

$$
I_{S C}=n e \mu E
$$

where $n$ is the density of charge carriers, $e$ is the elementary charge, $\mu$ is the mobility, and $E$ is the electric field, assuming lossless contacts. ${ }^{57}$ It should be noted that in equation 3 , mobility is a complex function of temperature, electric field, and polymer properties. Since the density of charge carriers is a function of a material system's response to illumination, and the electric field is set by the difference in work function between the cathode and anode, the mobility of charge carriers dominates the determination of short circuit current. Any current loss mechanisms will drive down the short circuit current; however, equation 3 offers insight to compare the internal physics of devices manufactured under similar conditions. The fill factor of a solar cell is a measure of how well the solar cell functions as a diode. The fill factor is given by:

$$
\text { Fill Factor }=\frac{P_{\text {Max }}}{J_{S C} V_{O C}}
$$

where is the maximum power density produced along the $\mathrm{J}-\mathrm{V}$ curve, is the short circuit current density, and is the open circuit voltage. Fundamentally this is the maximum power produced divided by the theoretical maximum power, which is the open circuit voltage multiplied by the short circuit current (Figure 9). 


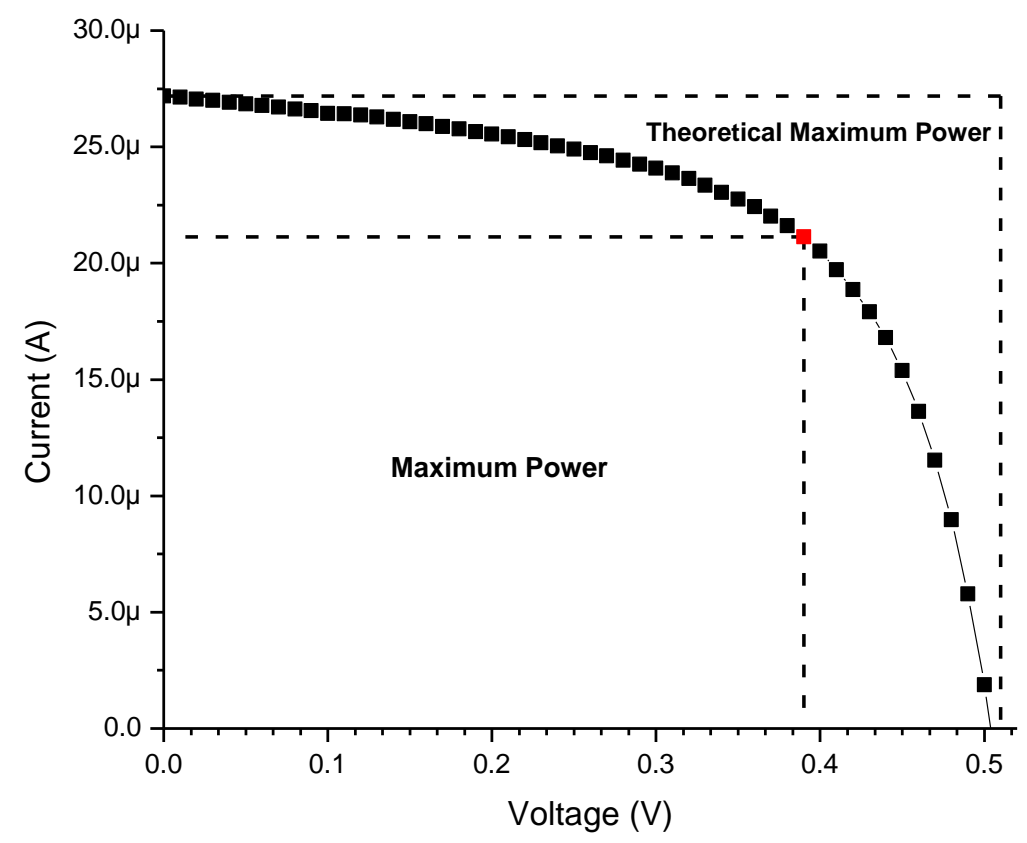

Figure 9: Illustration of the fill factor calculation, which is the ratio of the maximum power divided by the theoretical maximum power.

It is a measure of ideality of the solar cell, and is indicative of the ease of charge extraction within the solar cell. In polymer solar cells, charge extraction is a complicated process; however, higher fill factors are indicative of lower recombination rates and good charge carrier mobility. ${ }^{57}$

The maximum power a solar cell can produce is found by plotting the product of $\mathrm{J}-\mathrm{V}$ ordered pairs versus voltage. The maximum power point is useful in finding the power per unit area that can be produced as well as in the calculation of the fill factor. The maximum power is also used in calculating the power conversion efficiency of polymer solar cells by dividing the maximum power produced in the solar cell by the solar intensity incident to the OPV surface under AM 1.5G.

Power conversion efficiency is simply the ratio between the optical power incident to the solar cell and the electrical power produced by the solar cell. A high 
efficiency, while being the least indicative of any one specific phenomenon, is the best indicator of a good solar cell design because it is a direct measurement of the goal of any solar cell, to convert solar energy to usable energy.

Solar cells are also characterized by their absorption characteristics. For most silicon-based photovoltaics, the absorption characteristics are driven by surface modifications designed to reduce reflectivity. That reflectivity is driven by a large dielectric constant. OPVs have significantly smaller dielectric constants which complicates exciton separation processes, but creates very little reflectivity. As such, OPV absorbance is driven by the active layer quality and thickness. These absorption characteristics can be defined through optical density measurements. Optical density (OD) is a measure of the absorption of any particular wavelength and is given by:

$$
O D=-\log _{10}\left(\frac{I_{\text {Out }}}{I_{\text {In }}}\right)
$$

where $I_{\text {Out }}$ is the intensity of radiation passing through the cell, and $I_{I n}$ is the intensity of the radiation shining on the cell. For instance, a solar cell with an optical density of one would have a transmittance of 0.1 , meaning that $90 \%$ of the incident light was absorbed. A solar cell with an optical density of two would absorb $99 \%$ of the light falling on it. The construction of the cells described here allow for two passes through the material. Thus, an optical density of 1.0 would absorb $90 \%$ of the light through the first pass and $90 \%$ of the remaining light on the second pass, resulting in $99 \%$ total absorption. Although in practice, thin film interference effects between the reflective cathode and the polymer active layer decrease the total amount of light available on the second pass, meaning that we only know qualitatively that the actual absorbance is slightly larger than 
in a single pass. Thus, the optical density measurement, made on a per wavelength basis, becomes a powerful tool in gauging the amount of the spectrum that is useful to any particular system, no matter its complexity. Another consequence of the absorbance spectrum is the direct qualitative observation of crystalline "shoulders" of P3HT (Figure $10) .^{58}$

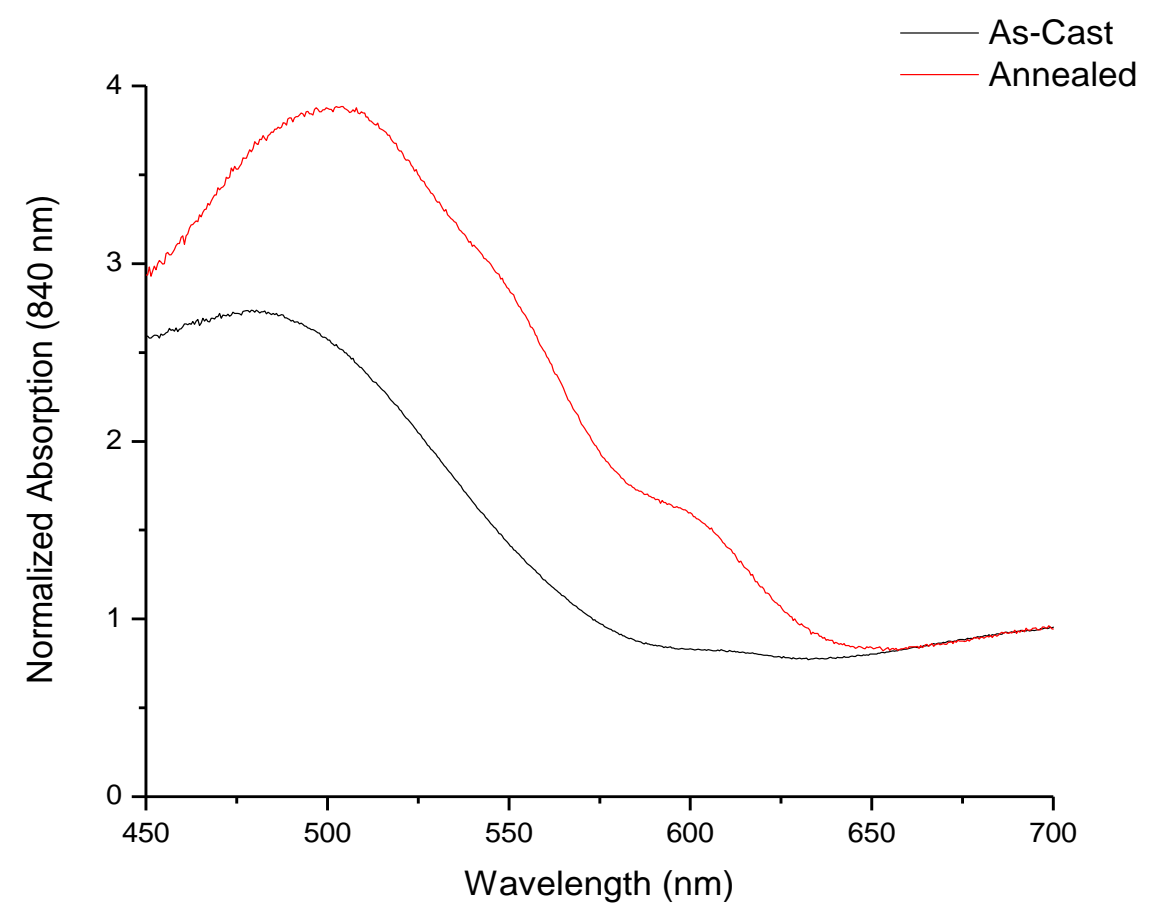

Figure 10: Representative absorption spectra for PCPDTBT-P3HT:PCBM devices before and after annealing, showing annealing inducing a red-shift in the main amorphous peak $(\sim 480 \mathrm{~nm}$ as-cast, and $\sim 510 \mathrm{~nm}$ after annealing) as well as crystaline shoulders at $\sim 540 \mathrm{~nm}$ and $\sim 600 \mathrm{~nm}$.

These crystalline regions exhibit lower energy, longer wavelength, "shoulders" in the optical density spectrum and the pronounced red shift in the main amorphous peak.

Finding the crystalline "shoulders" quantitatively is difficult, as the processed absorbance spectra always have some degree of noise associated with heat and stray light. This ambient noise, while not significantly evident in regular absorbance spectra, complicates the first derivative such that the exact location of the crystalline "shoulders" is difficult to 
obtain. Although this inability to find the exact location of the shoulder prevents a quantitative analysis, a qualitative analysis is still possible (Figure 11).

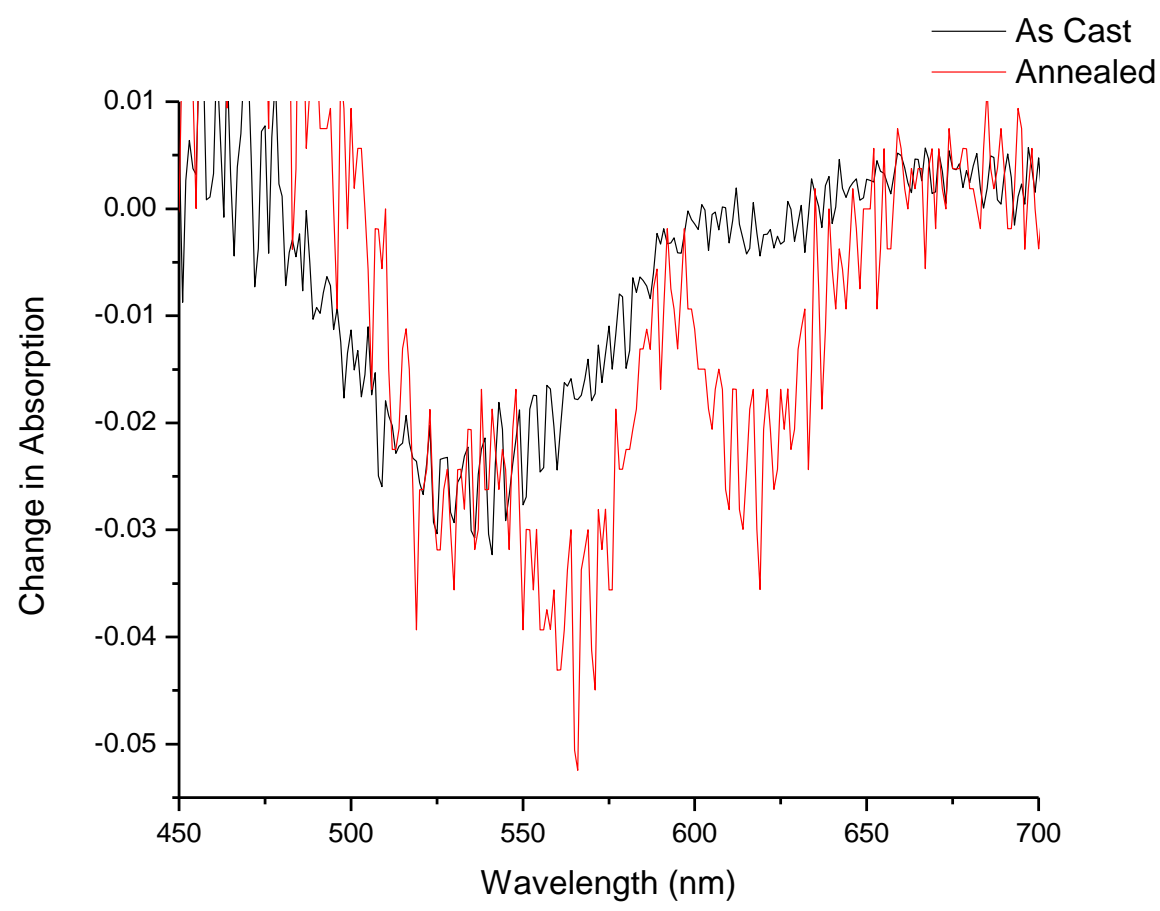

Figure 11: The first derivative of the simplified absorption spectra for a PCPDTBT-P3HT:PCBM device as cast and annealed showing the existence of "shoulders" at 550 and $600 \mathrm{~nm}$ in the annealed spectra that are not present in the as-cast film. 


\section{PROCEDURES}

In order to investigate the effects of QDs in an OPV system, four levels of QD loading were created: $0.25 \mathrm{mg} / \mathrm{mL}$ or $0.7 \mathrm{wt} \%, 1.0 \mathrm{mg} / \mathrm{mL}$ or $2.7 \mathrm{wt} \%, 4.0 \mathrm{mg} / \mathrm{mL}$ or 10 wt\%, and a control group with no QDs. The concentration value corresponds to the amount of QDs in the active layer while dissolved in chlorobenzene, while the weight percent corresponds to the assumed loading by weight within the active BHJ layer. Initial experiments revealed QD loading interfered with the morphology of the BHJ. Thus the effects of anneal temperature came into question, specifically would higher anneal temperatures offset the hindrances introduced through QD loading. As such, three anneal temperatures were investigated: the standard $110^{\circ} \mathrm{C}$ anneal, $140^{\circ} \mathrm{C}$, and an extreme $170^{\circ} \mathrm{C}$, all annealed for ten minutes. Each combination of QD loading and anneal temperature, or treatment, was given its own substrate patterned with ITO (grey) and aluminum (black rounded square outline) with four OPV pixel devices (purple) (Table I).

Table I: Visualization of Experimental Layout

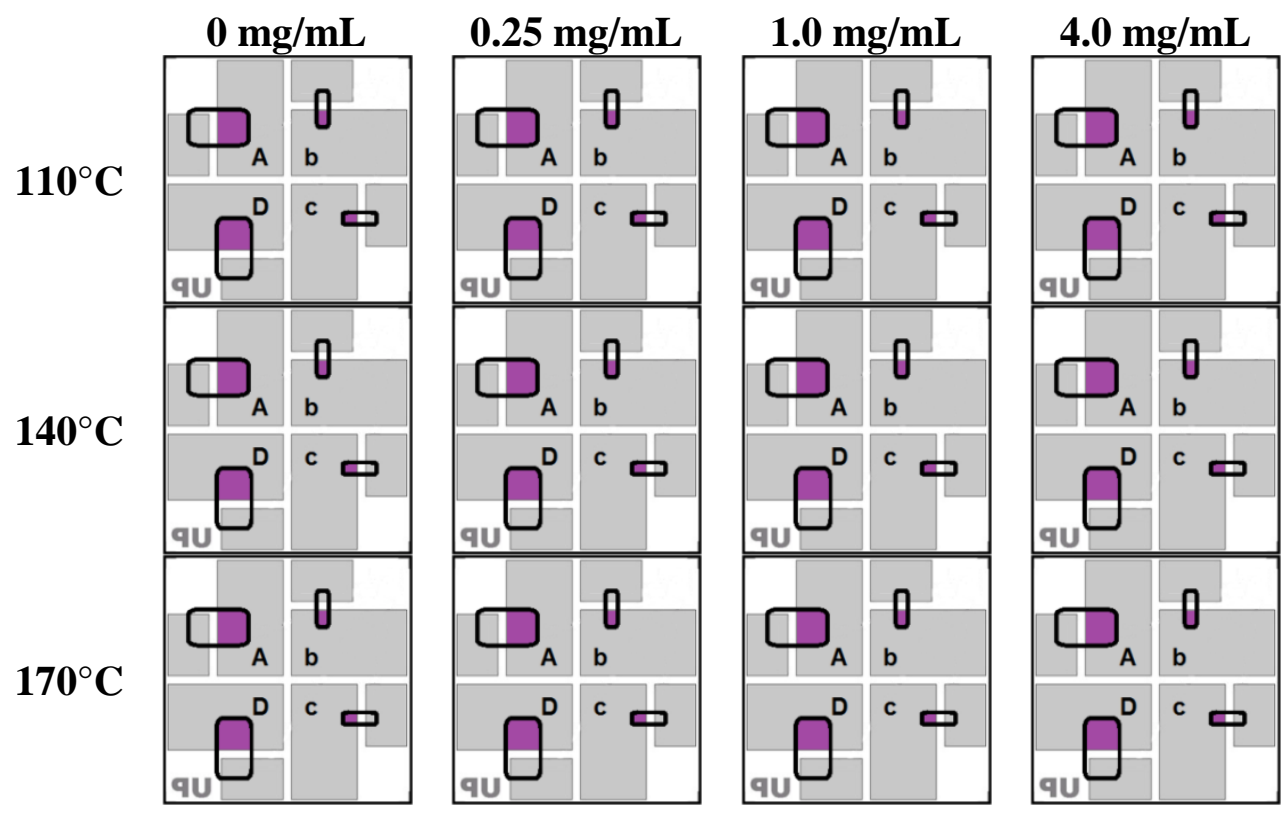




\section{Organic Photovoltaic Device Synthesis}

The devices were fabricated on $1 \mathrm{~mm}$ thick glass with a patterned indium tin oxide (ITO) layer approximately $125 \mathrm{~nm}$ thick (Figure 12).

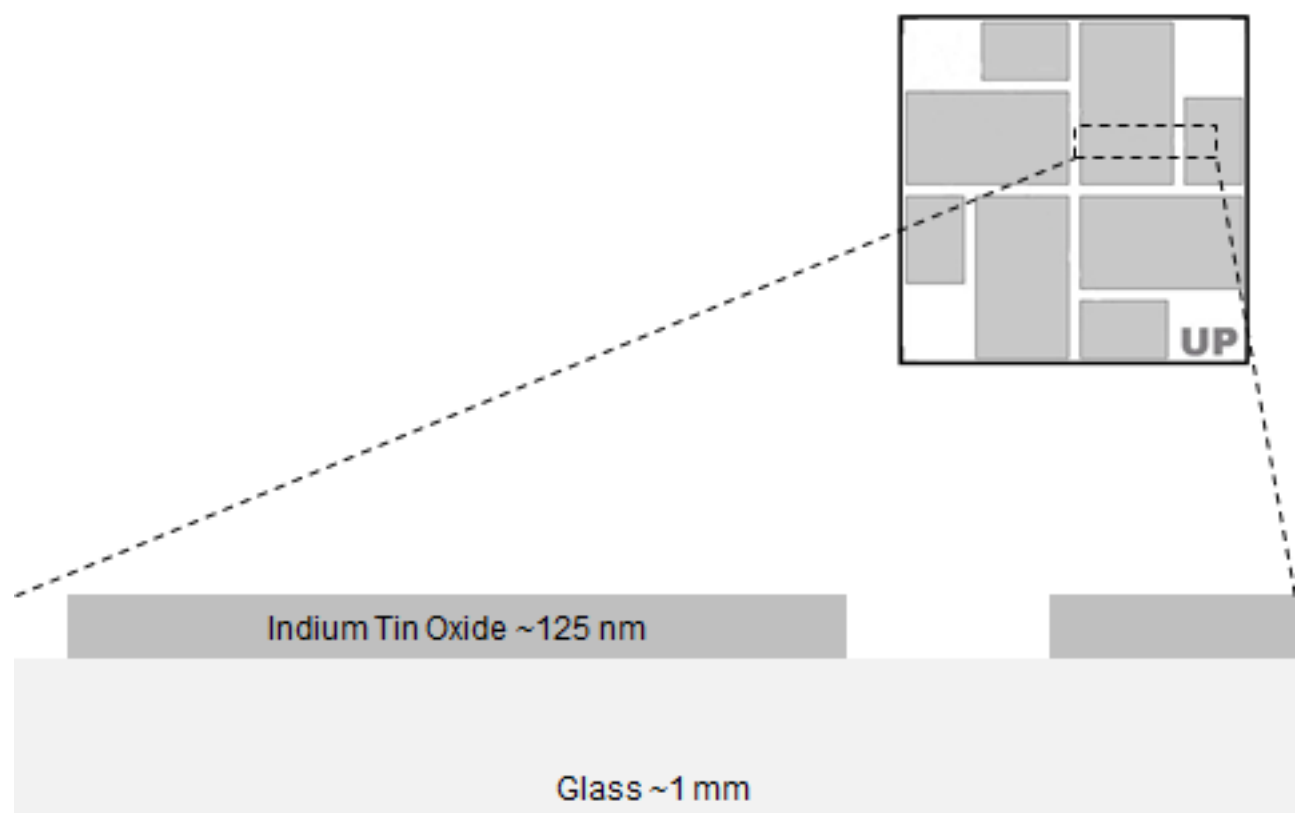

Figure 12: Schematic representation of the glass substrate with patterned ITO.

Both the glass and ITO are transparent to visible light so that the active layer of the device may be illuminated through the glass and ITO after the aluminum cathode is applied. The substrates were purchased from Colorado Concept Coatings LLC with the ITO applied and patterned. Scanning electron microscopy revealed the ITO coating to be smooth and without widespread defects.

\section{Cleaning the Substrates}

Particle contamination threatens the device with opportunities for open or short circuits between the metal cathode and the ITO anode. Short circuits would create a high leakage current in the pixel, and open circuits would decrease the overall area of the pixel and efficiency of the solar cell. Cleaning the substrates reduces the probability that these 
events will occur. The substrates underwent a multi-step cleaning procedure to reduce contamination of the photovoltaic devices. The cleaning procedure decreases the contamination already on the substrate and limits new contamination in a three-step process: initial inspection, acetone and isopropyl alcohol sonication baths, and UV-Ozone treatment. The substrates were inspected in a dust free area outside of the nitrogen glove box for blemishes in the ITO layer and visible particulate. Once through the initial inspection, the substrates were immersed and ultra-sonicated, first in a bath of acetone then in a bath of isopropyl alcohol with a compressed nitrogen drying step in between and after. Sonication in solvent baths, as opposed to a fluid streaming process, provides a higher energy cleaning process that increases the likelihood of dislodging particulate and chemical contaminants from the substrate. Acetone and isopropyl alcohol are both high vapor pressure solvents and evaporate completely from the substrate surface, leaving no residue. During the evaporation of the cleaning solvents, a low-pressure stream of nitrogen reduces the rate of recontamination by physically displacing dust. Once dried the substrates are ready for the last step in the cleaning process, UV ozone. The UV ozone process removes organic compounds, such as oils and skin cells, from the substrate surface. UV light activates ozone gas, dissociating organic material by forming $\mathrm{O}^{2}$ gas and reactive atomic oxygen. The UV ozone process also creates a hydrophilic condition on the substrates, which improves the uniformity of the applied polymer anode.

\section{Polymer Application}

Bulk hetero-junction polymer devices are unique in their construction. Since active materials are dissolved in solvents for dispersal, the creation of multiple layers must be carefully orchestrated to prevent subsequent layers of the device from dissolving the previous layers of the device. Multiple layers were achieved through alternating 
between polar and non-polar solvents. The polymer anode material is water-soluble; however, the presence of moisture within the device can decrease lifetime and overall performance so careful steps must be taken to limit any remaining water. The polymer anode material poly(3,4-ethylenedioxythiophene) poly(styrenesulfonate), or PEDOT:PSS, was deposited on the surface of the substrate through spin coating to planarize the surface of the substrate (Figure 13).
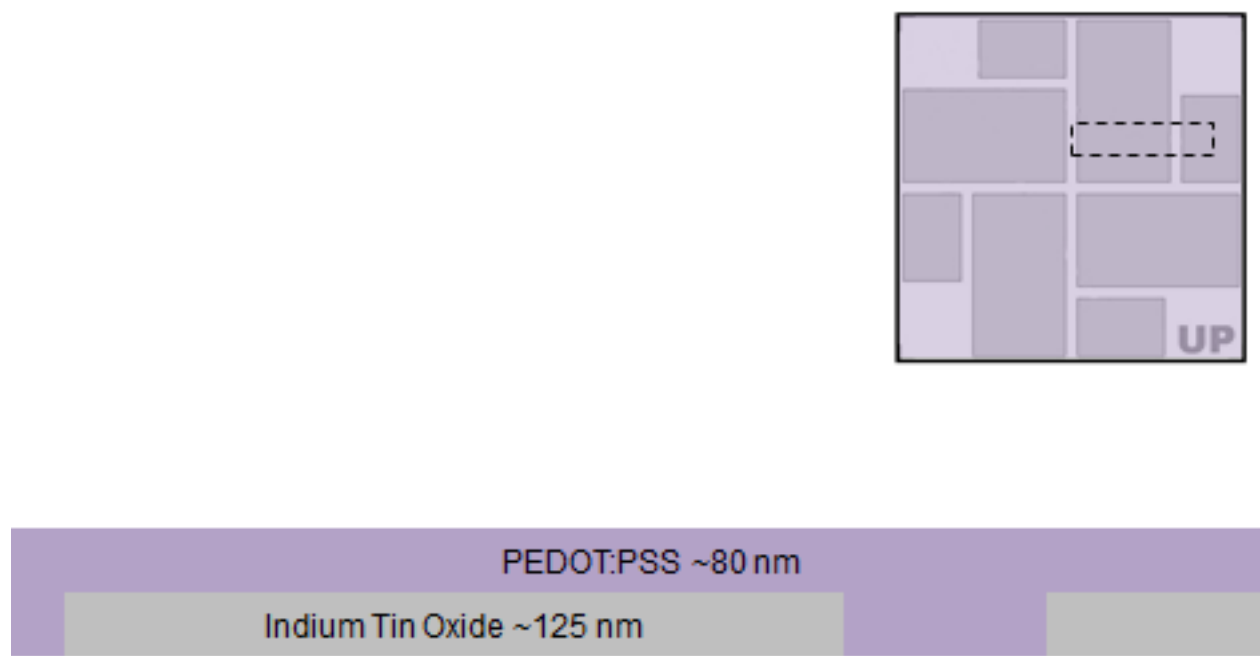

Glass $\sim 1 \mathrm{~mm}$

Figure 13: Schematic diagram of the ITO patterned glass substrate with a spin coated layer of PEDOT:PSS.

Using a buffer layer between the anode and the active material has several benefits that outweigh the possibility of water contamination in the active layer. First, the polymeric anode increases smoothness between the anode and active layer by providing a physical transition between inorganic and organic materials. Second, the electrical characteristics ease charge transfer and create better ohmic contact. ${ }^{60}$ Lastly, PEDOT:PSS effectively screens electrons from reaching the ITO anode. ${ }^{61}$ 
The PEDOT:PSS used in this study was CLEVIOSTM P VP AI 4083, from Heraeus in Leverkusen, Germany. The aqueous solution of PEDOT:PSS was spun on the substrates at $8000 \mathrm{RPM}$ for one minute then annealed at $125^{\circ} \mathrm{C}$ for 15 minutes to drive off any excess water. Since the applied PEDOT:PSS layer is conductive, sometimes as high as $0.2 \mathrm{~S} / \mathrm{m}$, the excess PEDOT:PSS can significantly contribute to leakage effects by bridging the anode and cathode of the solar cell. ${ }^{62}$ The leakage effects can be minimized by electrically isolating the small ITO pad, which is used as the cathodic landing pad for electrical testing, from the large anodic ITO pad. Thus, the PEDOT:PSS layer was removed from the outer edges of the substrates by wiping with a laboratory swab, and a continuous scratch was made around the small ITO pads to completely sever the PEDOT:PSS conductive pathways (Figure 14).
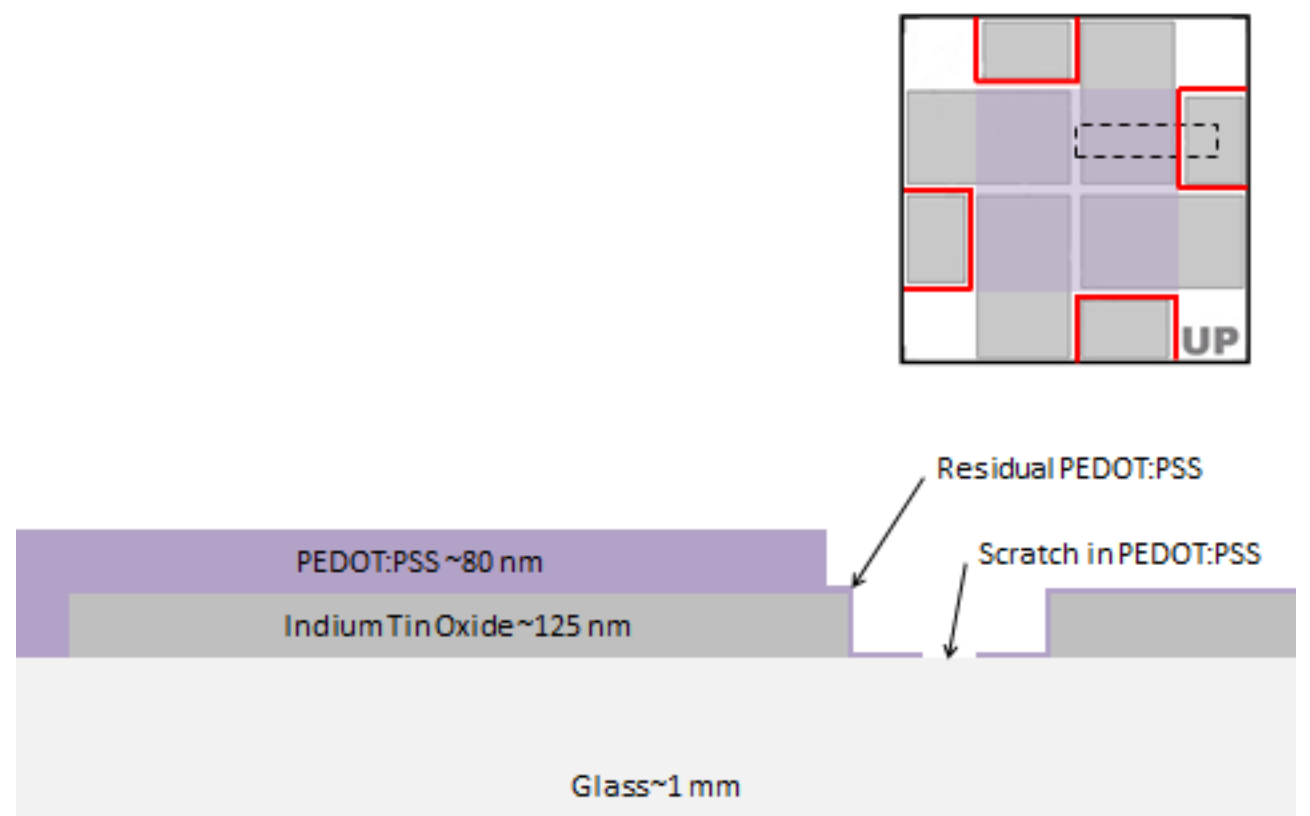

Figure 14: Schematic showing the remaining PEDOT:PSS layer, and the continuous scratches in red, both meant to electrical isolate the small ITO pad from the large ITO pad. 
Once the PEDOT:PSS is applied and patterned the substrates are ready to enter the nitrogen glove box. The nitrogen glove box is an inert atmosphere, pseudo-clean room environment that limits water vapor and oxygen content to below one part per million throughout fabrication and testing. If oxygen and water content are too high, the active polymer has the opportunity to photocorrode, and device performance will decrease dramatically. ${ }^{63}$ In order to maintain the inert atmosphere in the glove box, the substrates were placed in an evacuation chamber, pumped down to $10^{-3}$ torr and pressurized with the nitrogen atmosphere in the glove box. The evacuation procedure was repeated three times before the substrates were moved into the glove box.

Now inside the nitrogen glove box, the substrates with the patterned PEDOT:PSS layer are ready for active layer deposition. The active layer materials are mixed together and deposited simultaneously on top of the applied PEDOT:PSS layer (Figure 15).
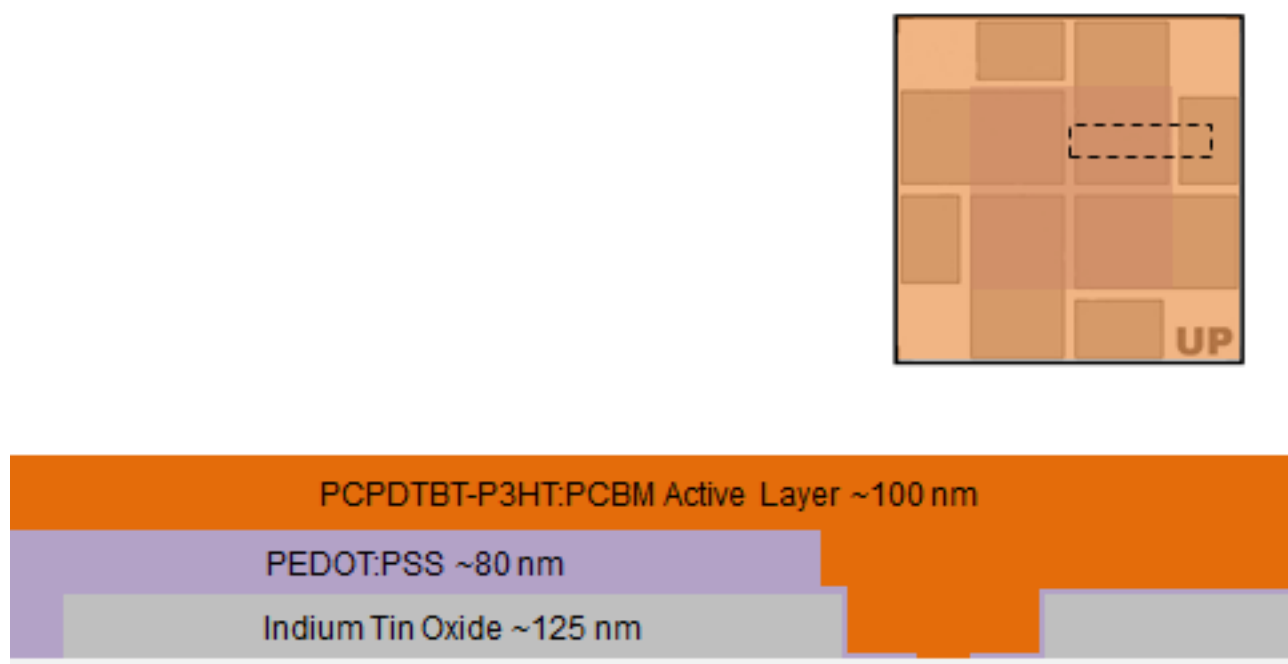

$$
\text { Glass } \sim 1 \mathrm{~mm}
$$

Figure 15: Schematic diagram showing the application of the active layer material on the patterned polymer anode and ITO. 
This is because bulk hetero-junction devices rely on self-assembly of beneficial morphologies, which reduces processing energy. The applied active layers are to vary in QD concentration and remain the same in P3HT, PCBM, and PCPDTBT ratio. To achieve this, each polymer, the PCPDTBT and P3HT, and the fullerene derivative, PCBM, were weighed out in a dry state in an ideal ratio of $20 \mathrm{mg}$ PCBM, $5 \mathrm{mg}$ ZZ50, and $20 \mathrm{mg}$ P3HT to every $1 \mathrm{~mL}$ of chlorobenzene to create a semi-concentrated stock solution. The concentrated stock solution was then diluted using quantum dot laden chlorobenzene, at a loading of $4.0 \mathrm{mg} / \mathrm{mL}, 1.0 \mathrm{mg} / \mathrm{mL}, 0.25 \mathrm{mg} / \mathrm{mL}$, as well a control group with no quantum dots to produce a ratio of $16 \mathrm{mg}$ PCBM, $4 \mathrm{mg}$ ZZ50, and $16 \mathrm{mg}$ P3HT to every $1 \mathrm{~mL}$ of chlorobenzene. This process creates four solutions that differ in quantum dot concentration without a difference in the ratio of active layer components. The addition of QDs changes the weight percent loading of the active layer components; however, it is assumed that the ratio of the donor material to the acceptor material is the driving force for performance. Thus, as long as the material ratio is constant between the devices, the active material variable is assumed constant.

Conjugated polymers rely on $\pi-\pi$ stacking to transfer charge between polymer backbones. This $\pi-\pi$ interaction, the same interaction that holds sheets of graphene together to form graphite, is so strong the polymers resist dissolution. To reduce this interaction, insulating side chains are added; however, this reduction must be carefully balanced with device performance since the side chains suppress conduction. This balance produces polymers that may be dissolved, but only under heated agitation for long periods. The semi-concentrated stock solution was set to stir for 24 hours at $500 \mathrm{rpm}$ 
and $50^{\circ} \mathrm{C}$ and the step was repeated for the final device solutions in order to facilitate complete dissolution. This step provides reason to believe the assumption that all polymers and nanoparticles (modified fullerenes and quantum dots) were evenly dispersed in the sense that no large "chunks" of polymer were deposited.

Once the active layer solutions are prepared, they are visually inspected for small "chunks" of material and a color shift from purple to orange to check for dissolution of the polymer components. The color shift is evidence of the polymer backbone coiling due to increased mobility in the strong solvent chlorobenzene. The dissolved solutions are pipetted onto substrates. Using the pipette, a small "bubble" is made to drag the polymer solution along the surface of the substrate. Once coated, the bubble is burst and the substrate is accelerated at $3000 \mathrm{RPM} / \mathrm{s}$ to $2000 \mathrm{RPM}$ to spin for 60 seconds.

After the semi-conducting active layer is deposited, the excess material must be removed from the outer edges of the substrate. Since the small ITO pad is used as the mechanically robust landing for the testing probes, Ohmic contact between the aluminum cathode of the solar cell and the small conductive ITO pad is required. If semi-conductive material separates the metal and ITO, it could function as a diode complicating the signal and ultimately driving down performance. To keep this from happening, we remove the active layer from the edges of the substrate using a laboratory swab dipped in tetrahydrofuran solvent (Figure 16). 

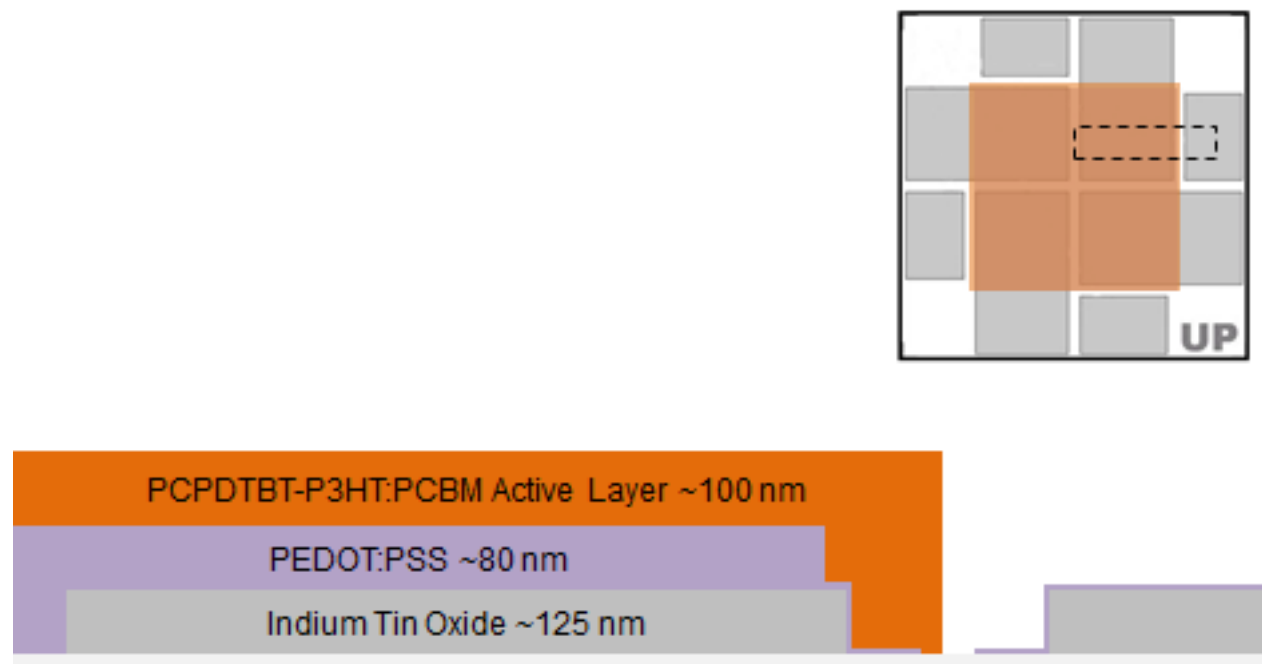

Glass $\sim 1 \mathrm{~mm}$

Figure 16: Schematic of the remaining active material (orange) removed from the edges of the substrate.

Once the solvent has evaporated from the devices, they are transferred to the "dry" side in the nitrogen glove box, so-called for its lack of evaporating solvents, for aluminum cathode deposition.

\section{Vacuum Evaporation}

Depositing the aluminum cathode on the devices required the use of physical vapor deposition, specifically by vacuum evaporation. In vacuum evaporation, a resistively heated metal either evaporates or sublimes until its vapor pressure is sufficiently large enough for deposition. A shadow mask, made by precision machining holes in an aluminum plate, patterns the deposited metal films by blocking certain areas to exposure, in exactly the same way as a stencil for spray paint. This "line of sight" deposition requires a high vacuum, between $10^{-6}$ and $10^{-9}$ Torr, to limit the interaction between the deposition material and gaseous molecules. 
The evaporation aimed to protect the active polymer layer by shielding the devices with a shutter while the aluminum melts, then applying a slowly deposited thin layer to shield the active layer from the fast application of the bulk of the electrode. Once the aluminum melted, indicated by a sharp increase in current, the evaporation needed to stabilize at a consistent and slow deposition rate, around $0.1 \AA$ As. At this slow and steady rate of evaporation, the evaporation shutter was opened, exposing the shadow mask and devices to the aluminum. After applying approximately $10 \mathrm{~nm}$ of aluminum, the deposition rate was increased to $\sim 1.0 \AA / \mathrm{s}$ in order to deposit the remaining $90 \mathrm{~nm}$ of material that would make the aluminum metal cathode (Figure 17).

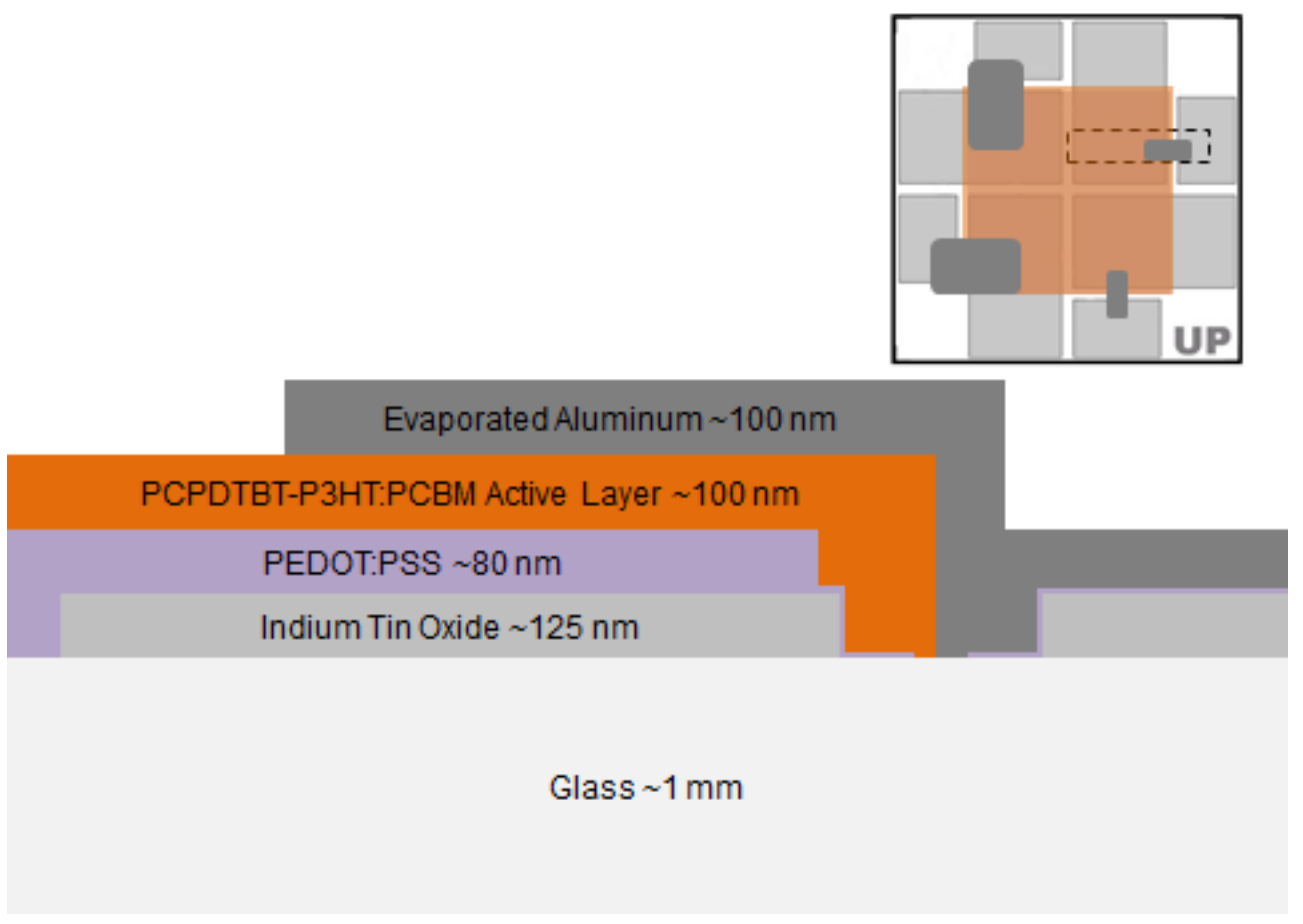

Figure 17: Schematic diagram of a finished OPV device showing the approximate geometry and thickness of the various thin films.

Although the active layer covers a large portion of the substrate, the actual OPV pixel area is between aluminum cathode material and anode material. It is important to note that the wiping procedures of both the PEDOT:PSS and active layer aim to electrically 
isolate the small ITO pad on the right of the schematic. Since it is a wiping procedure, there is an aim and an acceptable side to error. For the PEDOT:PSS layer we aim to wipe up to the edge of the large ITO pad with error on the side of wiping too much. On the other hand, we wipe the active layer to the middle of the gap between ITO pads and err on the side of wiping too little. This particular configuration of wipe and error ensures that the large anodic ITO pad always has a layer of semiconducting material between it and the small cathodic ITO pad. If this electrical isolation is not achieved, direct contact between the anode landing pad and cathode landing leads to shorting and drives device performance down.

\section{Testing}

Once fabricated, the devices were ready for testing in their as-cast state. Each OPV required optical and electrical characterization. In order to shield the results of the testing from uncontrollable factors, each testing group was randomized on the substrate level. This means that the order of the treatments, each combination of QD loading and anneal temperature, was randomized while the order of the pixels, A through D, was not randomized.

There were three sets of testing. In each set of tests, the absorption spectra were taken as well as the J-V curve with and without illumination. The first set of testing captured device performance before any annealing steps. The next set of testing involved electrical testing before and after annealing coupled with absorption data after the anneal step. The last set of testing was a detailed J-V curve, in which the step size between sourced voltages was reduced by a factor of five. This final test data was the data used in statistical analysis because of reduced variance from smaller voltage step sizes, which 
increase the accuracy of linearly interpolated values. Differences between the data sets, while present, were not attributed to degradation mechanisms. In fact, some devices improved over time. Since these improvements were present and degradation was not observed, these changes can only be attributed to long timescale diffusion events.

\section{Electrical Characterization}

The electrical characteristics of the OPV devices were recorded using a Keithley 2400 Sourcemeter in conjunction with LabView software, by sourcing voltage and recording current between $-0.5 \mathrm{~V}$ and $1.0 \mathrm{~V}$ in steps of $10 \mathrm{mV}$. Plotting these values as ordered pairs produces a current-voltage curve ( $\mathrm{J}-\mathrm{V}$ curve), which yields a plethora of information (Figure 18).

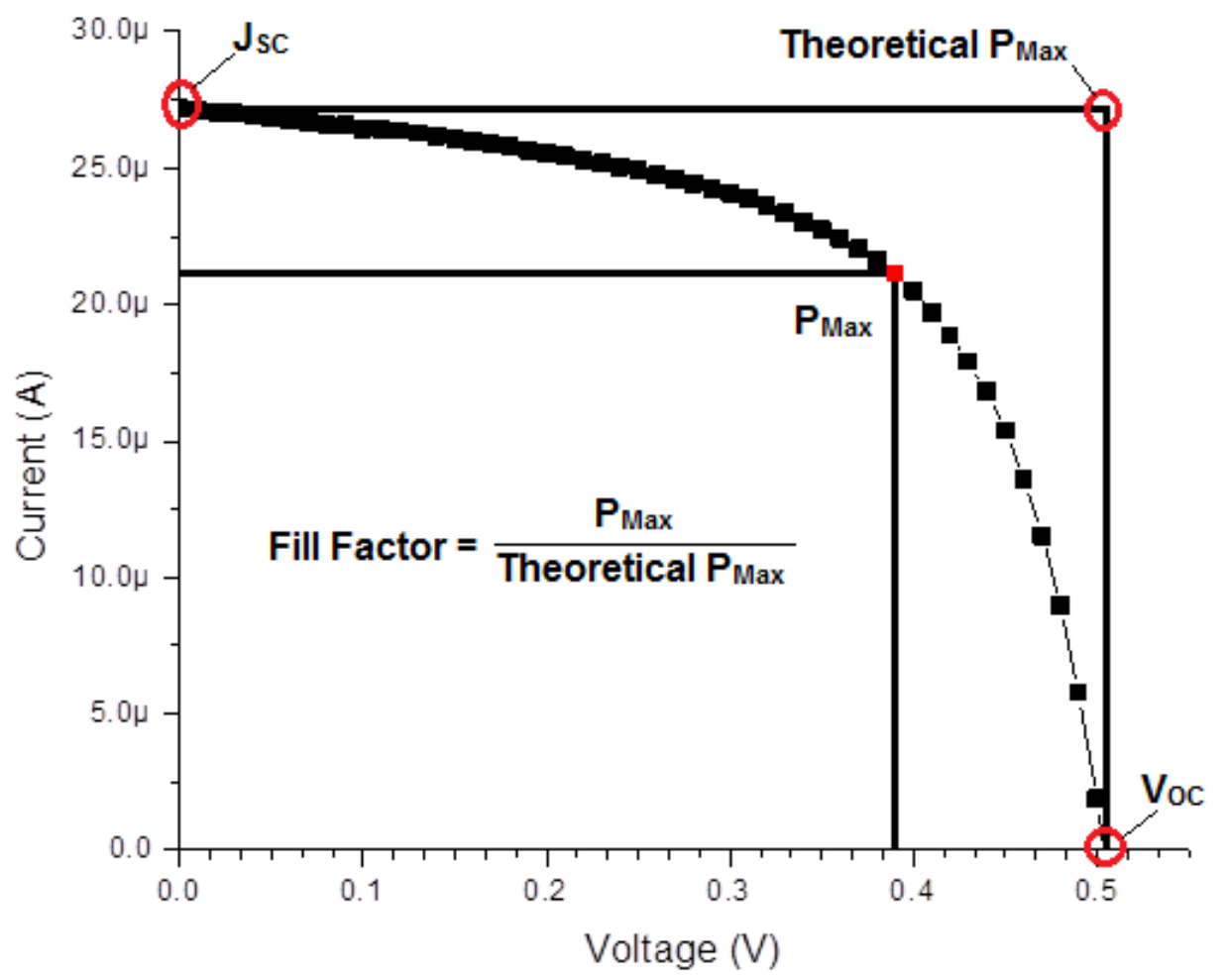

Figure 18: A typical J-V curve focused on the power-producing region of the curve with the maximum power point in red, illustrating the origin of four electrical characteristics of a solar cell. 
These J-V curves were fed into a program, which automatically analyzes each curve for open circuit voltage, short circuit current, fill factor, and power conversion efficiency. The value for short circuit current can be pulled directly from the data set; however, the open circuit voltage value was calculated through linear interpolation. This approximation is acceptable given the typically large slope and small voltage step size. The maximum power, which was not reported by the program but used in calculating fill factor and power conversion efficiency, was taken simply as the largest product of any current voltage pair. Plotting both current and power versus voltage shows that this approximation is also acceptable given small voltage step sizes (Figure 19).

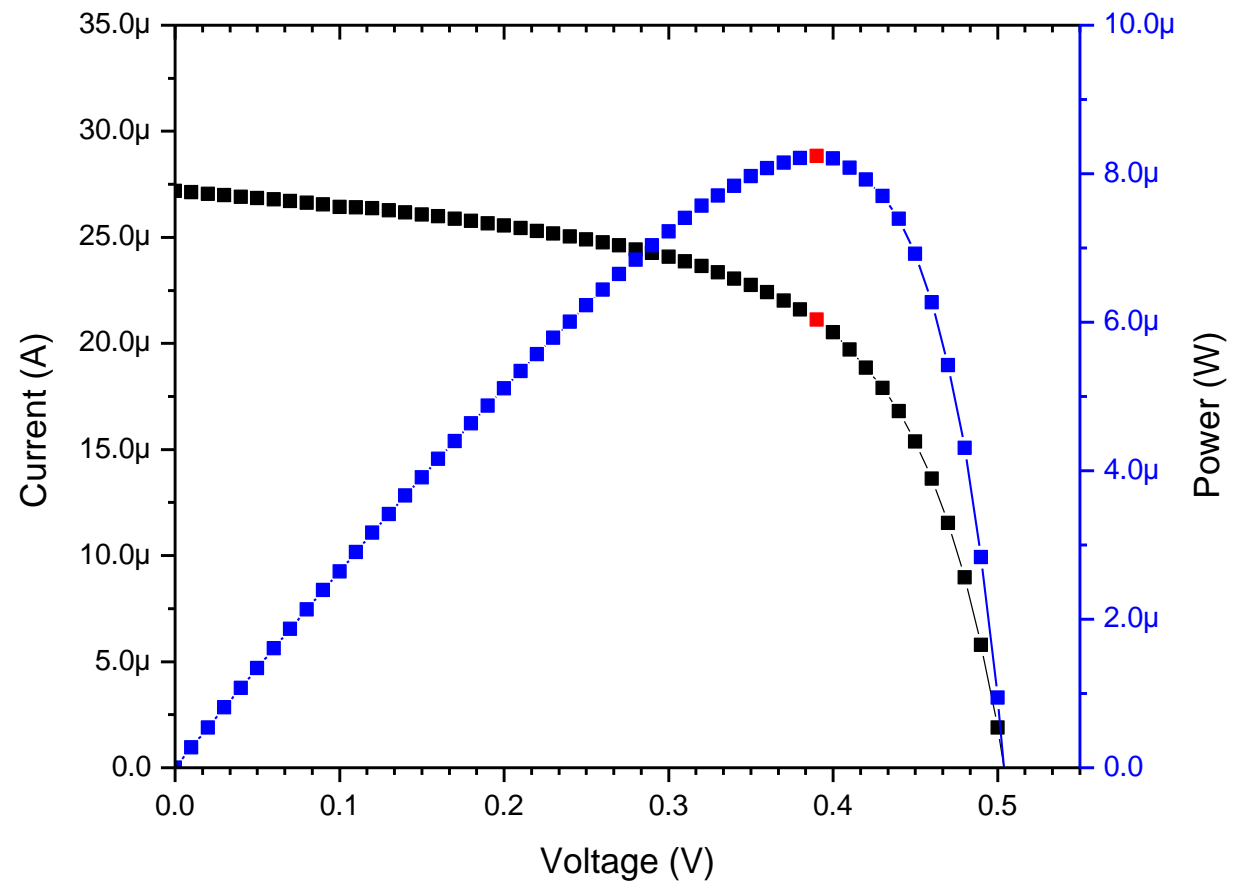

Figure 19: Current and power plotted against voltage, illustrating the resolution of the approximation for maximum power.

The other electrical characteristics, shunt and series resistance, were estimated through a linear regression of five data points. By taking the inverse of the slope near the open circuit voltage point and multiplying by the area of the pixel of interest, an 
approximate value of the series resistance can be calculated. The shunt resistance was calculated similarly, but instead used the points nearest the short circuit current.

The physical interface between the OPV and the testing apparatus was made through pogo pins in a static configuration and applying pressure from above (Figure 20).

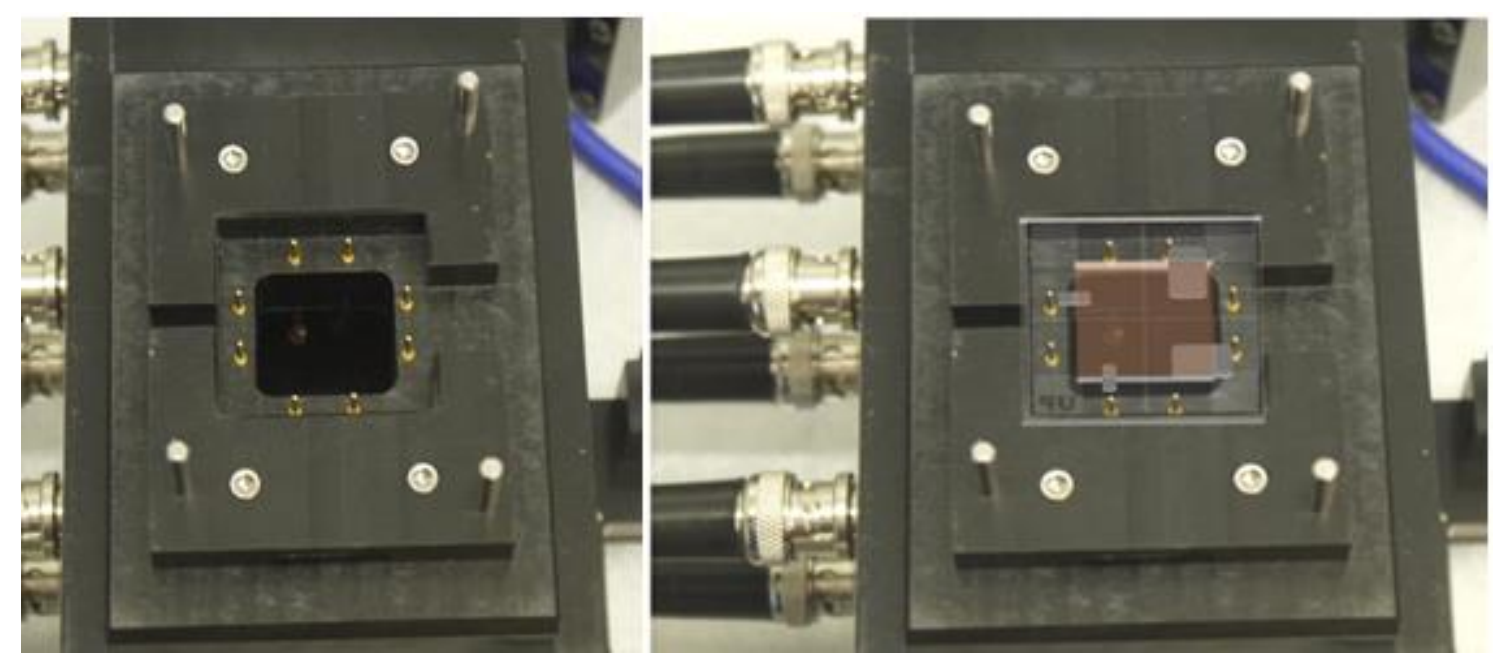

Figure 20: Electrical characterization jig, showing the eight pogo-pin connections required to test four OPV devices.

Since multiple tests are generally required to characterize the OPVs, the pogo pins connect to the large and small ITO pads rather than connecting to the large ITO pad and the thin aluminum cathode. While this testing method introduces a small resistance in the best scenario, the worst scenario being electrical shorts between anode and cathode with a non-Ohmic contact between the small ITO pad and the aluminum, it creates the mechanical durability required for multiple repeatable characterization steps.

\section{Optical Characterization}

Capturing the wavelength dependent absorption characteristics of the active layer was accomplished with an Ocean Optics HR2000+ UV-Vis spectrometer in line with an Ocean Optics LS-1 tungsten halogen lamp. The spectra were recorded in Ocean Optics SpectraSuite with an integration time of one millisecond and fifteen runs to average. The 
Sony ILX511B linear silicon CCD array used in the spectrometer can collect spectra between 200 and $1100 \mathrm{~nm}$ wavelengths. ${ }^{64}$ Since optical testing was done inside of the box, the substrates are held in place between two fiber optic cables that lead to output connectors that connect to the spectrometer and the light source (Figure 21).

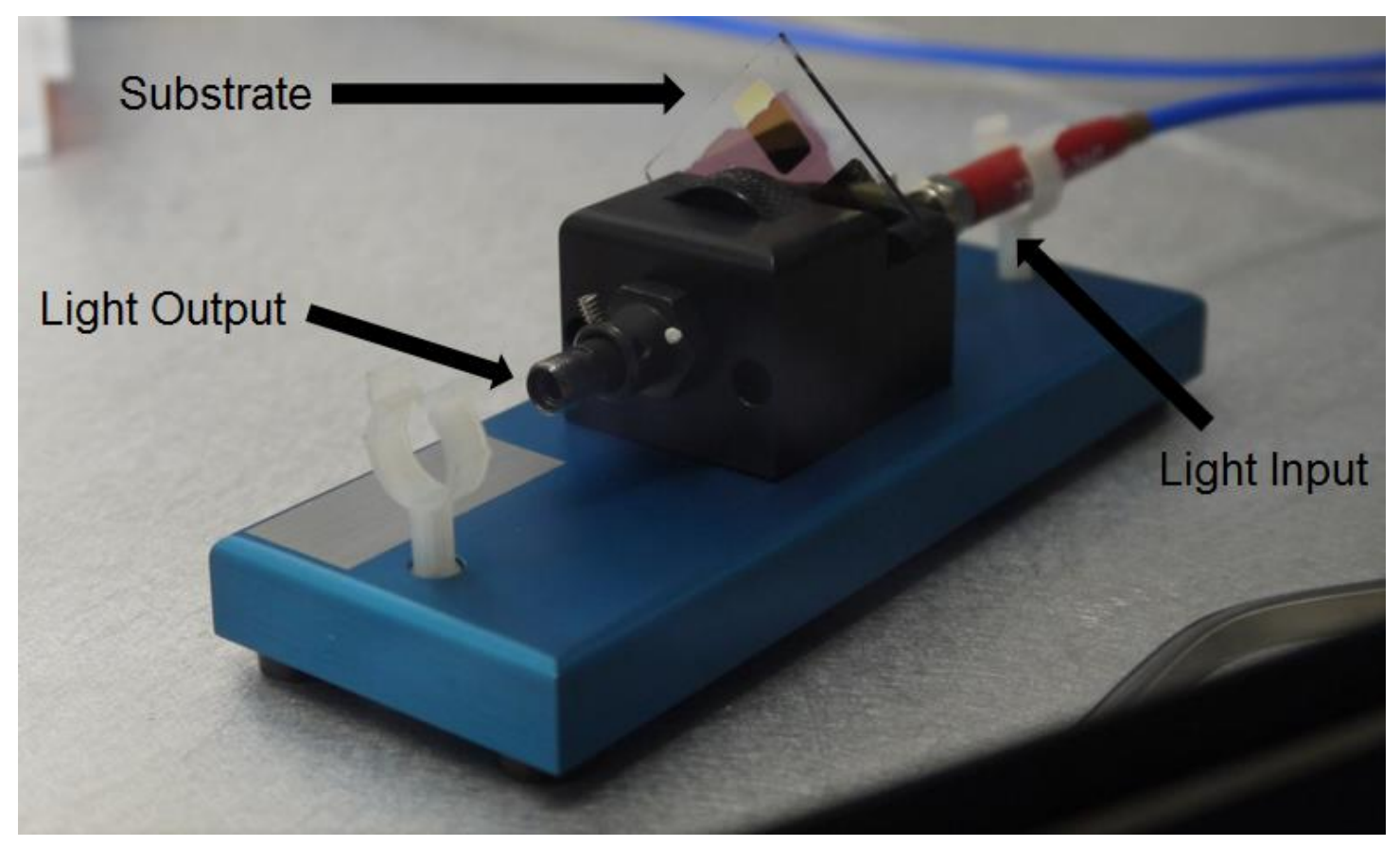

Figure 21: UV-Vis substrate holder inside of the nitrogen glove box environment.

We isolate the optical properties of the active layer by inserting a PEDOT:PSS covered substrate, and taking that as the light reference. Once referenced, the two spectra for that device are taken, one near pixel $b$ and the other near pixel c. Given the construction of the devices, with two large pixels on the left and two small pixels on the right, there are only two areas with enough active layer that are not covered with the aluminum anode. As such, the absorbance spectra for pixels $\mathrm{b}$ and $\mathrm{c}$ are not directly recorded and the analysis relies on the assumption that the active area of the pixel is 
similar in morphology to the area sampled for absorbance. Thus, each device yields two absorption spectra based on area not covered by the pixels (Figure 22).

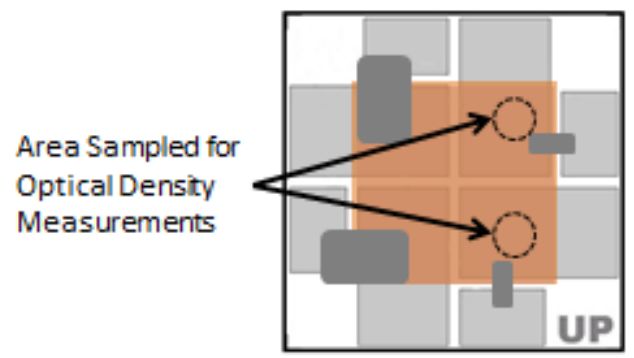

Figure 22: Schematic representation of the area of active layer sampled for optical density measurements.

It is important to note that the UV-vis spectroscopy is limited by the input spectrum (Figure 23).

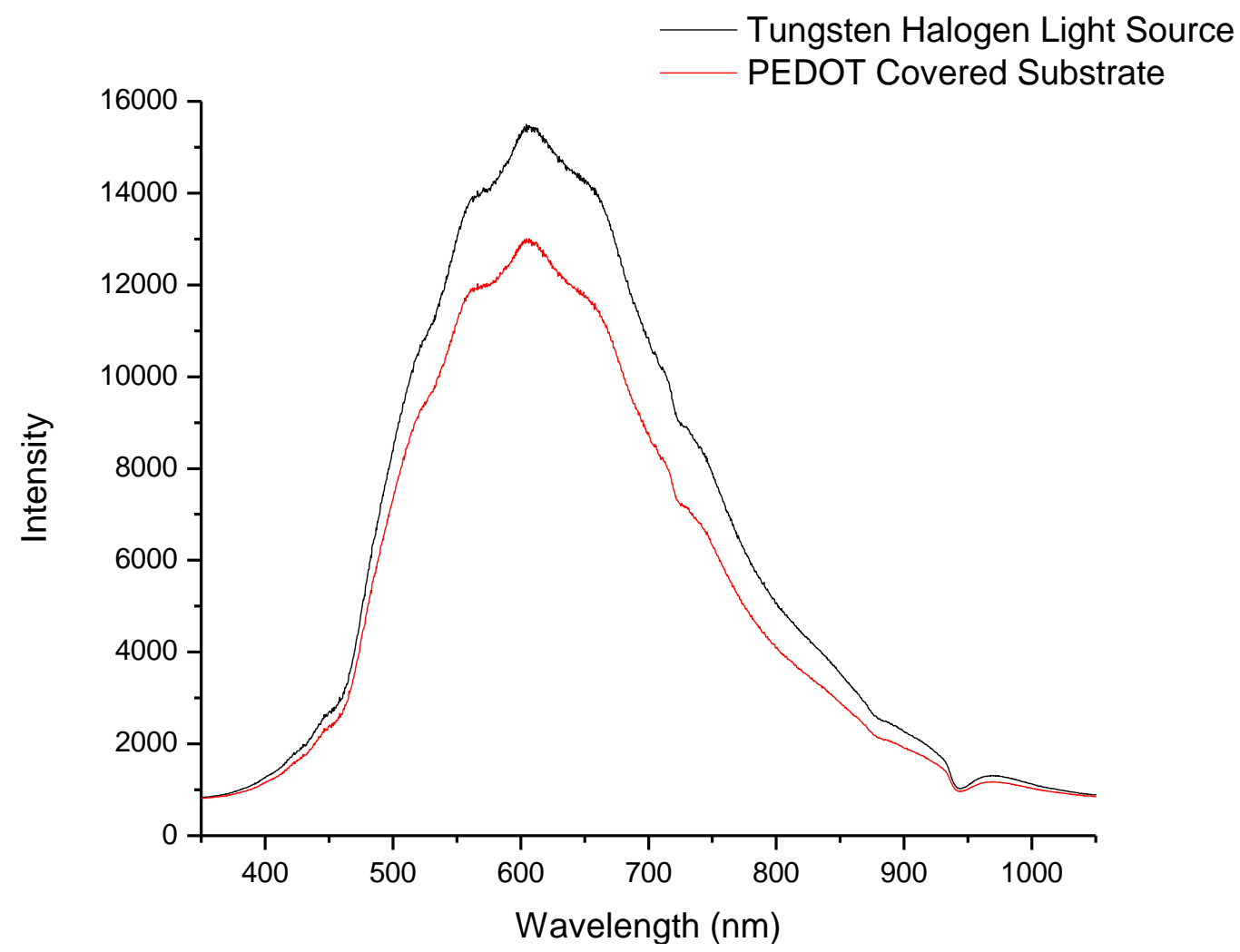

Figure 23: Input light spectrum for the UV-Vis data collected showing low intensity on the edges of the spectrum. 
The input spectrum, in this case, was created by a tungsten halogen light source; however, the actual input spectrum to the active layer is the original spectrum minus the reflectance off the surface of the substrate minus the absorbance of the glass and PEDOT:PSS.

The limitation stems from lower signal to noise ratio at the edges of the spectrum. As the signal across the spectrum is collected, a non-wavelength dependent level of noise is also captured. In intensity versus wavelength plot, that noise is represented in a slight jagged quality of the plotted signal and is relatively constant. Constant noise does not interfere with qualitative observations on intensity; however, since absorption is a logarithmic ratio between the given signal and the stored light reference, the noise evident in the observed spectra is no longer constant (Figure 24).

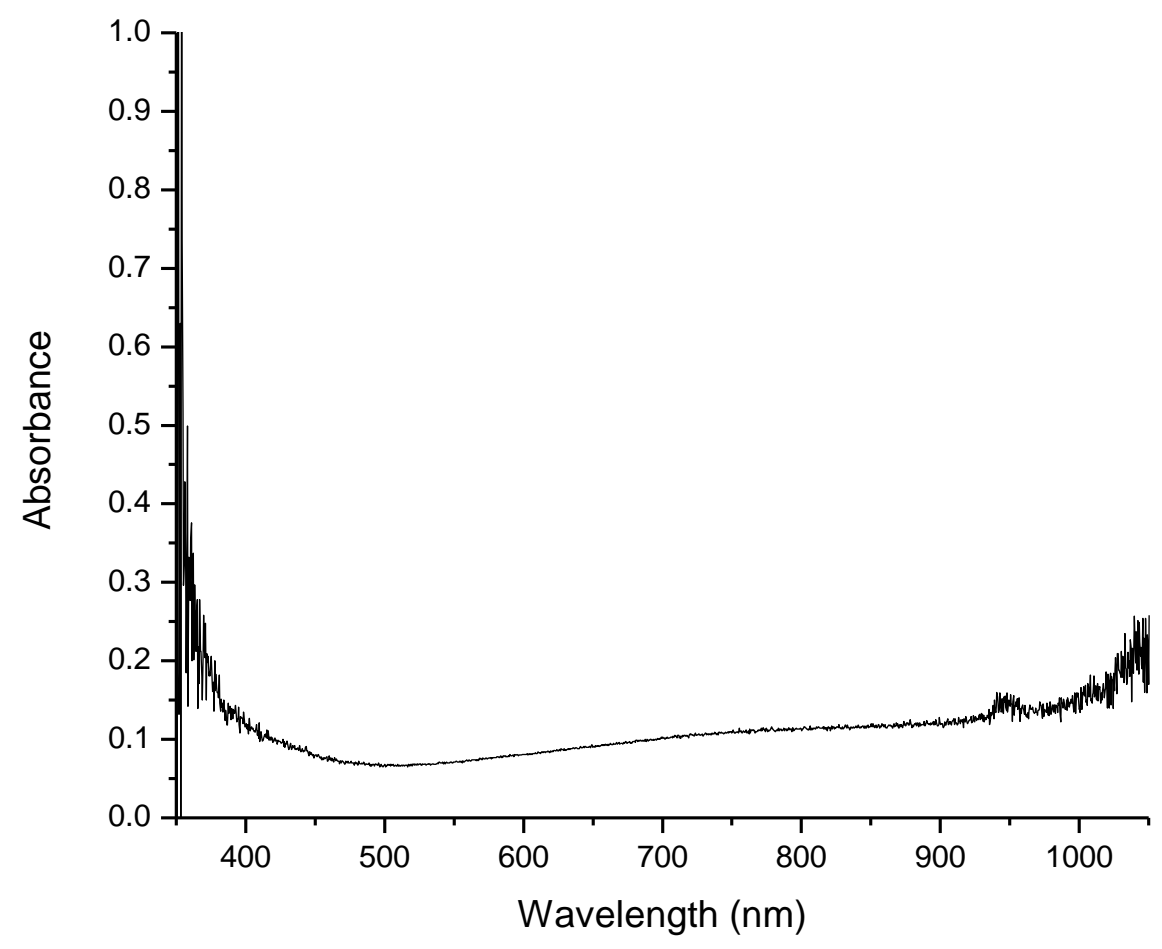

Figure 24: Absorbance of the PEDOT:PSS layer and the substrate showing decreasing signal to noise ratio on the edges of the spectrum. 
This is because the mathematical comparison introduces sensitivity to the signal to noise ratio. The practical implication is a qualitatively useful absorbance spectrum within the range of $\sim 400 \mathrm{~nm}$ to $\sim 900 \mathrm{~nm}$, and increasing noise outside that range. 


\section{RESULTS}

The statistical testing used in this analysis is an analysis of variance (ANOVA) method called the general linear model (GLM). The GLM is a powerful and adaptable model that relates a response, like fill factor or efficiency, to multiple input variables in a linear combination. Similar to any statistical testing method, a GLM relies on assumptions. The biggest assumption is that each treatment, or combination of input variables, must have equal variance with the other treatments. To test if these assumptions are met, we must plot the standardized residuals, the predicted value minus the actual value, against the fits of the characteristic equation (Figure 25).

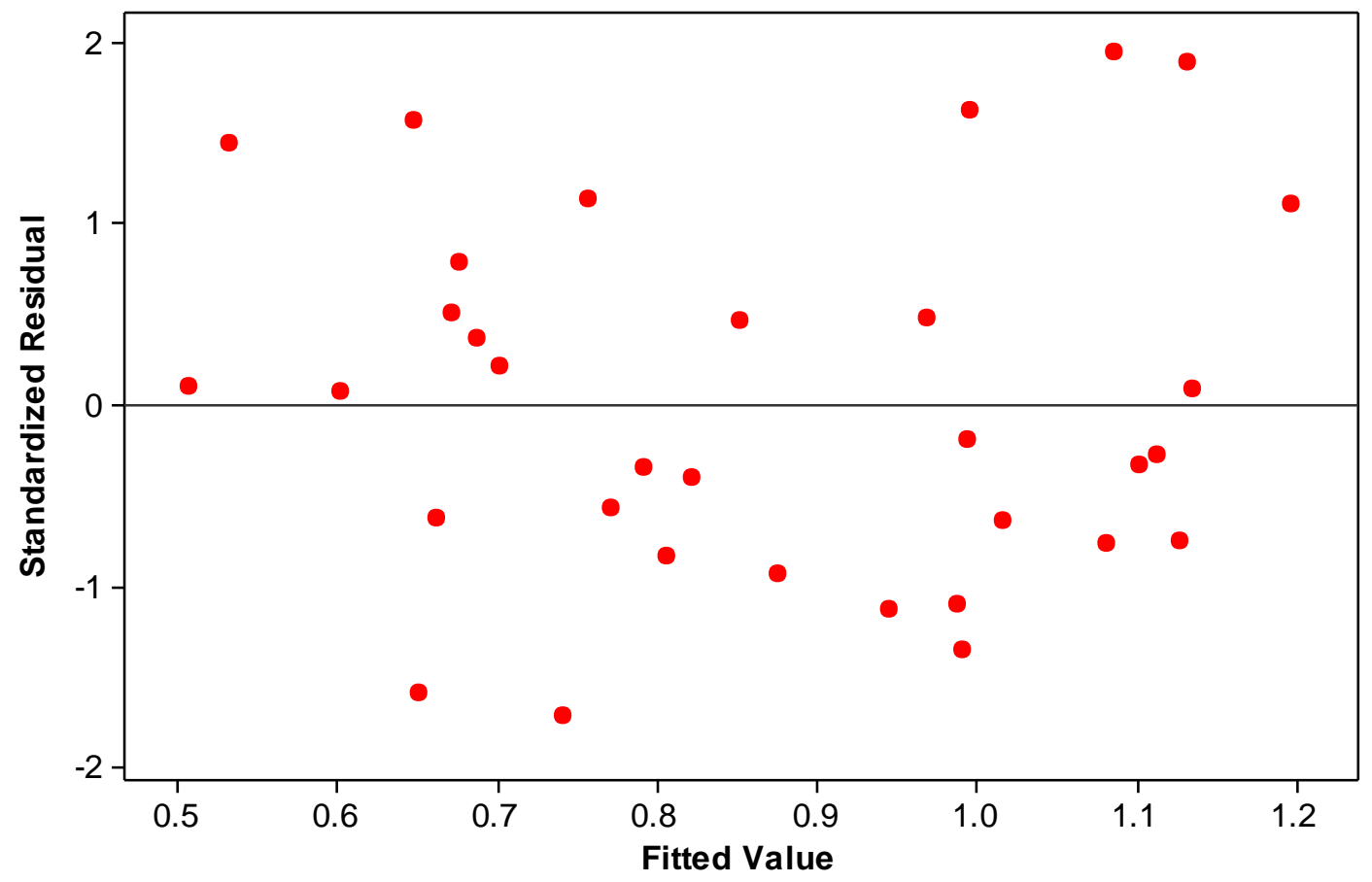

Figure 25: Standardized residuals versus the fitted values from the characteristic equation modeling the error found within the GLM model.

The residuals are a measure of error, as in how well the estimated equation fits with the data used to estimate it. Alone, the residuals are useful in finding outliers and detecting if 
the statistical model used is the best model for the job. When the residuals are plotted against the fitted values, the error is pinned to where it occurred in the model. This assigns error into a specific location within the theoretical model and allows for the detection of patterns in error, which mean that there is some signal not accounted for by the model. Without pinning the error to a space within the model, we can simply detect if the error falls into a normal distribution or not. With the pinning, we can differentiate between normally distributed error that appears randomly and normally distributed error from a signal not accounted for in the model. If the plot resembles a random assemblage of points, then the model fits the data.

The next assumption is that the data are normally distributed. The test for normality simply plots the standardized residuals on a log-log scale versus the percentage of data that fall into that residual, and applies some sort of normality test, like AndersonDarling or Kolmogorov-Smirnov, rating how much each point varies from the line representing normality (Figure 26).

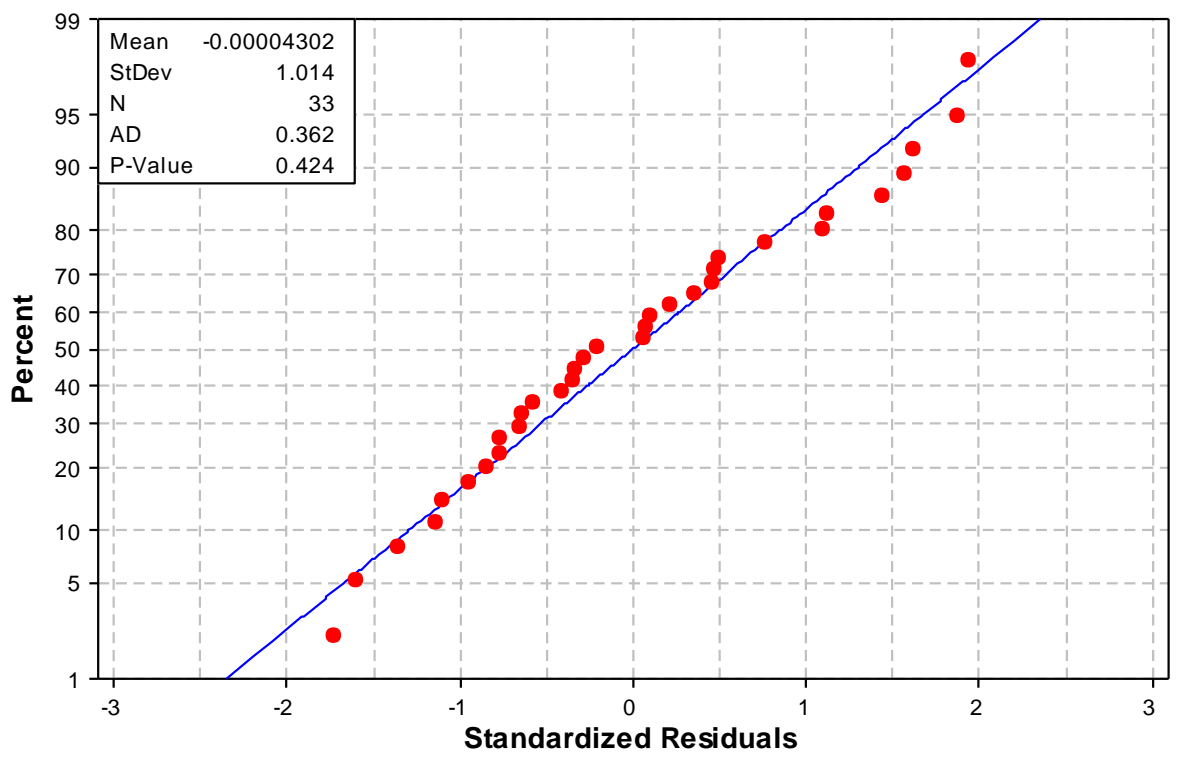

Figure 26: Standardized residuals plotted against the percent of data that have the associated residual value, visually representing the normal distribution of residuals from the model. 
The last method, which is really more of a pre-treatment of data, is the dot plot. The dot-plot simply plots the response variable on the $\mathrm{x}$-axis within groups according to input variables. This allows direct observation of the variation within each treatment and an initial estimation of outliers and, as such, is included with the observed results.

The data collected stem from 12 substrates, each with four pixels labeled A-D. Pixel A and D are both "big" pixels with an approximate area of $42 \mathrm{~mm}^{2}$, while pixel B and C are "small" pixels with an approximate area of $3.75 \mathrm{~mm}^{2}$. The input variable of QD loading had four levels while the input variable of anneal temperature had three levels, meaning that each substrate underwent a unique treatment, giving four pixels at each combination. Since the four pixels give the four responses at each treatment, and the pixels are physically interrelated, this study contains pseudo-replicates rather than actual replicates. Pseudo-replication can lead to p-values that are too small because of correlated errors. In this study, the pseudo-replication was taken into account by modeling pixel effects. Through modeling pixel effects, the overall power of the experiment is decreased which ensures accurate p-values.

Each response variable is given 48 values; however, the variance of the highest level of quantum dot loading proved starkly different in most response variables. This difference was large enough to disrupt the model, and thus the analysis was repeated without the responses for the $4 \mathrm{mg} / \mathrm{mL}$ QD loading. Each response then dropped to 36 data points, minus outliers.

Whenever discussing an experiment, and its associated statistics, it is useful to discuss the power of the experiment. The most accessible way to discuss power is simply to be aware of the degrees of freedom in the test. There are two competing aspects 
involved, the number of data points we have that represents our stockpile of information, and the input variables that we would like to model that represents the knowledge that we would like to glean. The degrees of freedom required to model an input variable, like temperature or QD loading, is defined as one minus the level of that variable. The degrees of freedom provided, our stockpile, is given by one minus the number of data points in the model. If the degrees of freedom required by our model outnumber the degrees of freedom given by our data, we simply cannot afford that model. We need our stockpile to outnumber our model, because the balance is applied to modeling random error. Further the larger that balance, the better we can model random error, the more robust the resulting model. Modeling three levels of anneal temperature and thee levels of quantum dot loading takes four degrees of freedom, with another three degrees of freedom for modeling pixel effects. The interaction between quantum dot loading and anneal temperature takes another four degrees of freedom. Thus, the model requires either eleven or seven degrees of freedom, depending on if the interactions are statistically significant, while the remainder model random error. In the worst case, three outliers removed and modeling the interaction between QDs and annealing temperature, 21 degrees of freedom remain to model error with eleven degrees of freedom to model responses.

Overall, the addition of QDs decreased performance of the OPVs. In the case of the $4 \mathrm{mg} / \mathrm{mL}$ loading, the performance decrease was severe enough to merit removal from the statistical analysis. Also, anneal temperatures higher than $110^{\circ} \mathrm{C}$ showed no added benefit or a marked decrease in overall performance. 


\section{Annealed Devices}

The electrical characteristics of a solar cell can become quite involved. There are several response variables present in one $\mathrm{J}-\mathrm{V}$ curve, each with its own implications. Six were found to fit within the GLM: Short Circuit Current Density $\left(\mathrm{J}_{\mathrm{SC}}\right)$, Open Circuit Voltage $\left(\mathrm{V}_{\mathrm{OC}}\right)$, Fill Factor (FF), Power Conversion Efficiency (PCE), and Series Resistance. Shunt resistance did not fit in the model; however, a dot-plot revealed strong evidence of an area based effect, which will be discussed in detail later.

Since the GLM was based in ANOVA, the output contains a value known as the F-Statistic. The F-Statistic is the ratio of variance between treatments to the variance between treatments. In essence the F-Statistic is quantitative expression of just how much a response variable's variation varies from the random error. Thus larger F-Statistics are indicative of stronger observed effects. Since the overall effect of adding QDs was a decrease in performance, a summary table of the F-Statistics shows where that performance decrease comes from (Table II).

Table II: F-Statistic Values for Statistically Significant Effects on Each Response

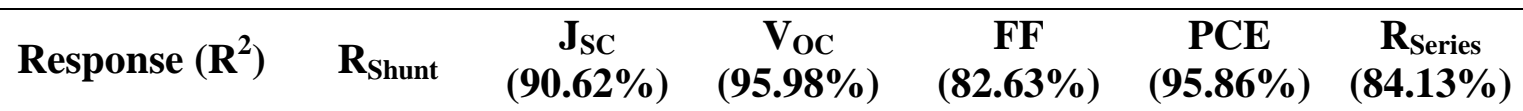

Quantum Dot Concentration

N/A

3.77

121.37

55.78

73.02

15.30

Anneal

Temperature

N/A

92.57

103.53

3.62

160.52

49.65

Pixel

Observed

25.97

Not Not

Significant Significant

13.56

Interaction of Quantum Dot Concentration and Anneal Temperature

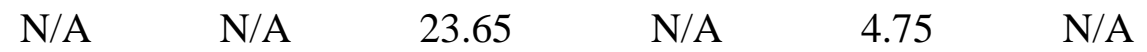




\section{Shunt Resistance}

The shunt resistance is generally a measure of the manufacturing process. Poor processing results in defects that allow leakage and recombination to become more dominant. Leakage pathways and recombination sites within the solar cell result in power loss, which lowers the power conversion efficiency of the solar cell. Thus, a large shunt resistance is beneficial to solar cells. In polymer solar cells, lower shunt resistance is understandable in as cast films because of large "chunks" of a difficult to dissolve polymer. Generally shunt resistance is improved with an anneal step as the heated polymer is more free to move and will likely "heal" defect regions and recombination sites. In this case, smaller pixels consistently show lower shunt resistance, despite normalization to area (Figure 27).

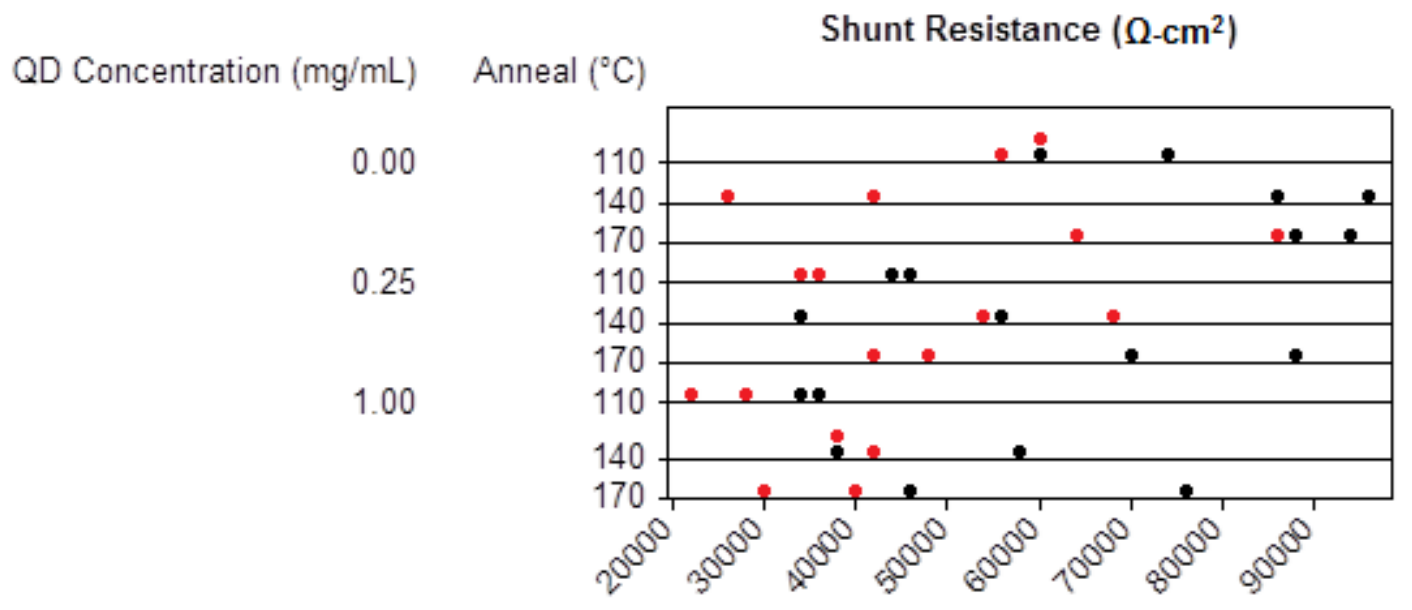

Figure 27: Dot-Plot of shunt resistance values showing small pixels, in red, with consistently lower shunt resistance, despite normalization to area.

Any difference in defects between experimental groups could either be a result of the material system used or processing error. Since the processing is done on a per-substrate basis with four pixels per substrate, and the treatments are done on a single substrate in 
random order, the differences in processing and material system should not be different between pixels.

The consequences of an area based error in a study with varying pixel sizes are propagations of the error in any measurement sensitive to area, or any calculated value that includes such measurements. Since an equal number of large and small pixels balanced the error, general trends are still observable. Unfortunately, any area-based trends are either overshadowed by, or falsely represented in, the area error.

\section{Short Circuit Current Density}

When the solar cell is illuminated the voltage difference between the electrodes of the solar cell is zero, the solar cell is said to be in short circuit conditions. The short circuit current density $\left(\mathrm{J}_{\mathrm{SC}}\right)$ is a measure of how much current a solar cell can pass under illumination, divided by the surface area of the solar cell. Larger currents are better from a performance point of view as they are indicative or larger photogenerated current, lower recombination rates, and good charge extraction. The data contained no obvious outliers (Figure 28).

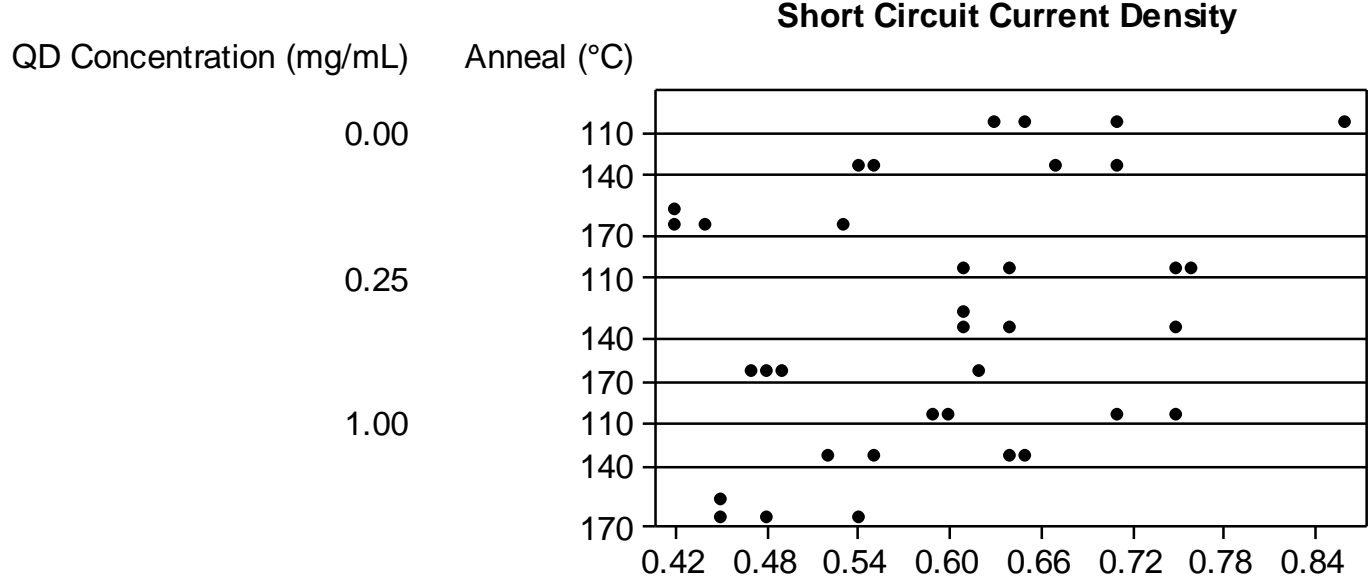

Figure 28: Dot-Plot of short circuit current density showing relatively equal variance on the device level. 
The $\mathrm{J}_{\mathrm{SC}}$ did not show a statistically significant interaction between anneal temperature and QD concentration. Because of this, the model was run without the interaction represented, meaning that $\mathrm{J}_{\mathrm{SC}}$ response was modeled using seven degrees of freedom, and the experimental error was given 27 degrees of freedom. The General Linear Model followed the data closely, giving an R-squared value of $90.62 \%$. This means that the $90.62 \%$ of the observed variation fits within the model. Since the only difference between pixels is their location on the substrate, and the response from each pixel was normalized to area, the variation between pixels gives a sense of the variation inherent in the testing process. The F-statistic offers a comparison between each parameter's contributions to the model while the P-value offers a probability that the observed effect simply happened by chance. While the QD concentration effect is statistically significant from random chance, this effect was an order of magnitude below pixel effects which was an order of magnitude below the effects of anneal temperature (

Figure 29, Table III). Given this, it becomes obvious that in this study, the anneal temperature was the driving factor in short circuit current density. Other conclusions could be drawn if the variation between pixels could be attributed to some other effect like edge length or defect size.

Table III: Test Statistics and P-Values for Short Circuit Current Density

\begin{tabular}{lcc}
\hline \multicolumn{1}{c}{ Effect $\left(\mathbf{R}^{2}=\mathbf{9 0 . 6 2 \%}\right)$} & F-Statistic & P-Value \\
\hline QD Concentration $(\mathbf{m g} / \mathbf{m L})$ & 3.77 & 0.035 \\
Anneal Temperature $\left({ }^{\circ} \mathbf{C}\right)$ & 92.57 & 0.000 \\
Pixel & 25.97 & 0.000 \\
\hline
\end{tabular}




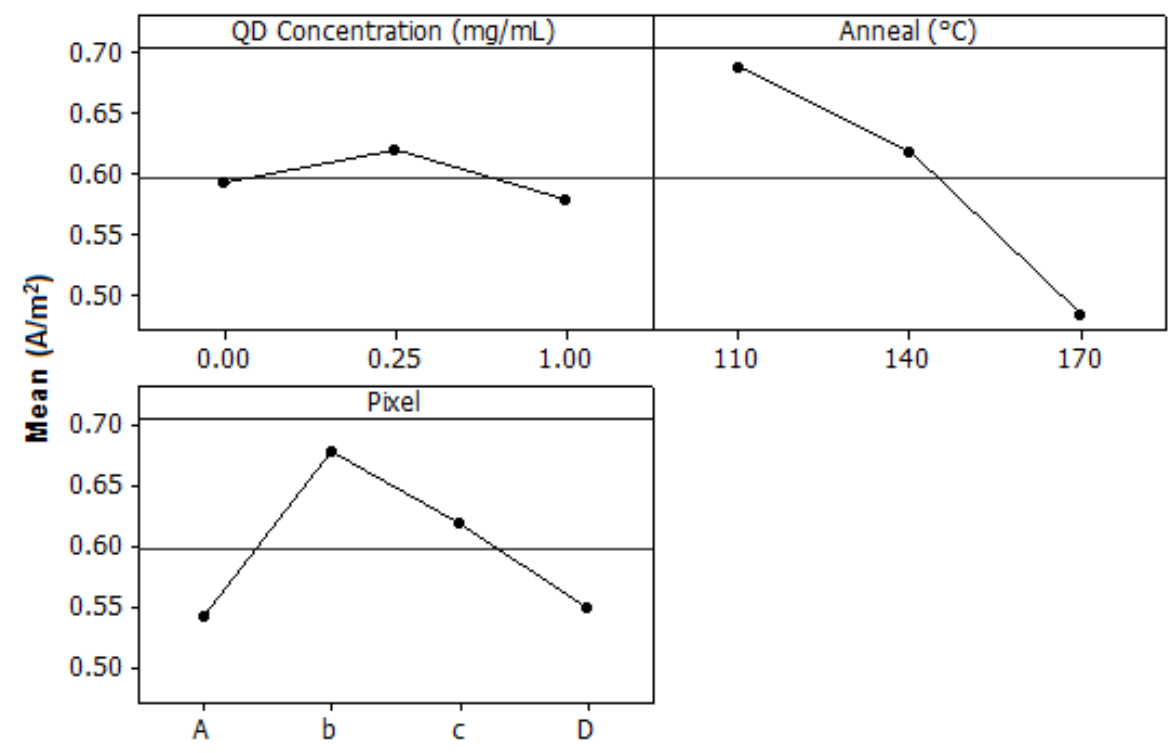

Figure 29: The main effects on the mean of short circuit current density, which show a large pixel variation with smaller pixels (b and c) performing more effectively, and a statistically significant effect from QD concentration much smaller than the observed pixel effects.

\section{Open Circuit Voltage}

The open circuit voltage data collected exhibited relatively equal variance between treatments and only one outlier was removed (Figure 30).

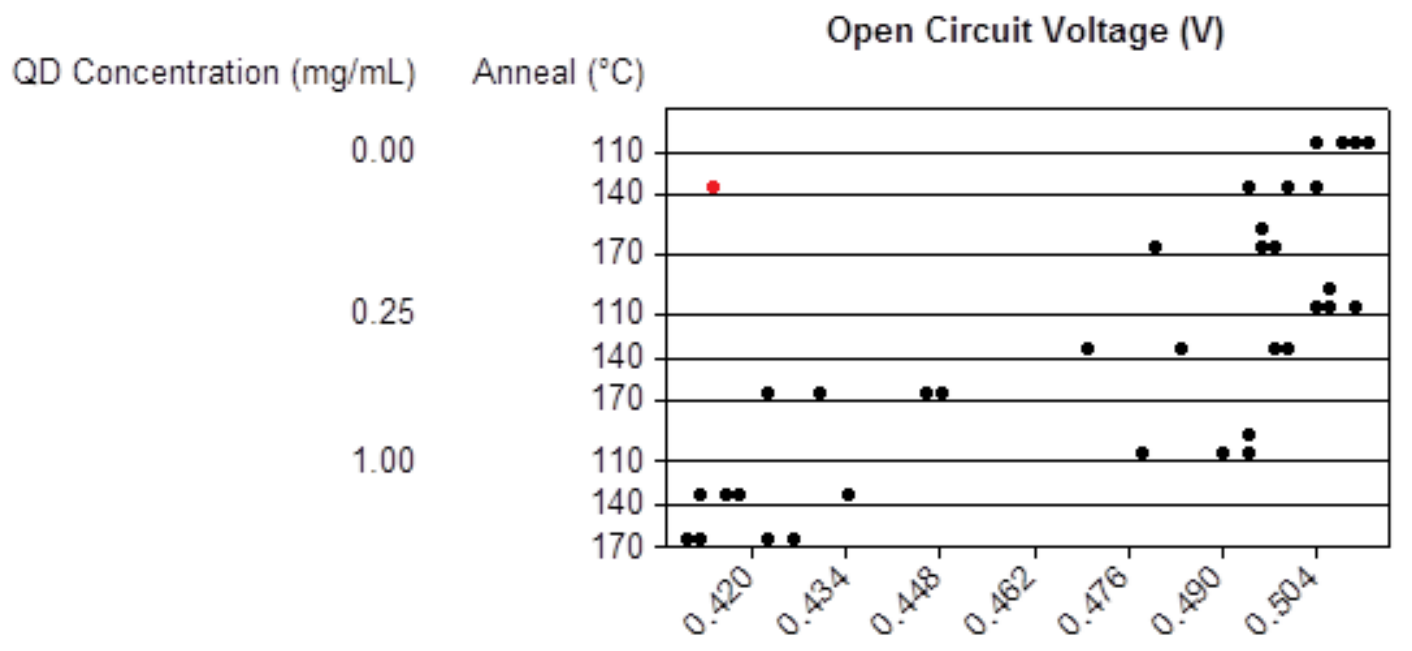

Figure 30: Dot-Plot of open circuit voltage with one removed outlier in red.

The GLM returned an $\mathrm{R}^{2}$ of $95.98 \%$ meaning that the model contains $\sim 96 \%$ of the observed variation. The model also rejected pixels effects at the $95 \%$ confidence level with a P-Value of 0.619. Unlike short circuit current density, the interaction between QD 
concentration and Anneal temperature was found to be statistically significant, meaning that a synergistic effect between to two input variables was observed. This extent of this effect, which can be gauged by its F-Statistic, was an order of magnitude lower than either effect alone (Table IV).

Table IV: Test statistics and P-Values for Open Circuit Voltage.

\begin{tabular}{lcc}
\hline \multicolumn{1}{c}{ Effect $\left(\mathbf{R}^{\mathbf{2}}=\mathbf{9 5 . 9 8 \%}\right)$} & F-Statistic & P-Value \\
\hline QD Concentration $(\mathbf{m g} / \mathbf{m L})$ & 121.37 & 0.000 \\
Anneal Temperature $\left({ }^{\circ} \mathbf{C}\right)$ & 103.53 & 0.000 \\
Pixel & 0.60 & 0.619 \\
$\begin{array}{l}\text { Interaction of QD Concentration } \\
\text { and Anneal Temperature }\end{array}$ & 23.65 & 0.000 \\
\hline
\end{tabular}

The interaction plot offers a qualitative aspect to the observed effects. Distinct slopes between lines indicate that the optimal anneal temperature varies between QD loadings. There are two distinct slopes present: a gradual slope evident throughout the anneal treatments for the devices lacking QDs, and a steep slope evident in parts of the devices containing QDs (Figure 31).

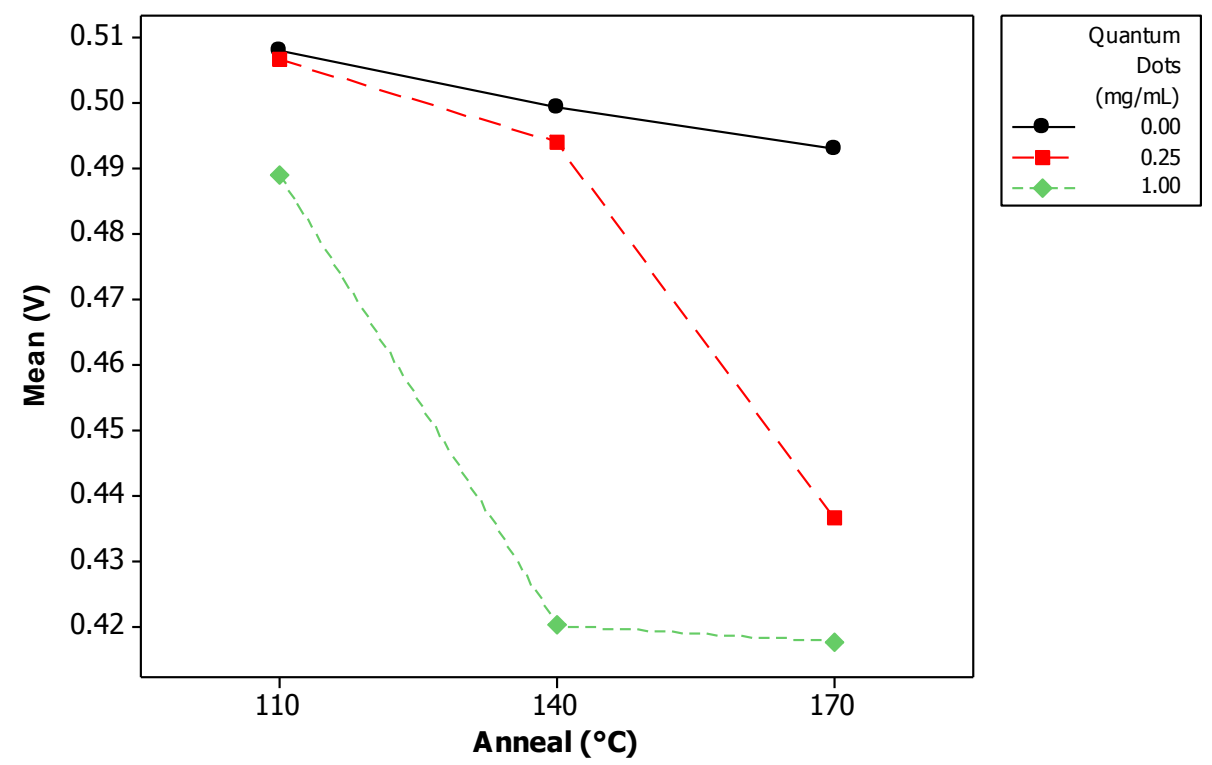

Figure 31: Interaction plot for the mean of open circuit voltage 
The open circuit voltage in a polymer photovoltaic is limited by the LUMO-HOMO offset between acceptor and donor respectively. The open circuit voltage can also be affected by the LUMO-LUMO offset between donor and acceptor materials and the work function of the top electrode. The LUMO level of PCBM increases with crystallinity due to expansion of the number of states within the band. Assuming the scope of P3HT HOMO level expansion fits with the slope evident in the devices without QDs and since the VOC from devices with QDs are substantially lower than the devices without quantum dots, the QDs are likely increasing the extent of P3HT crystallinity or disrupting the flow of charge through the solar cell.

\section{Fill Factor}

The fill factor (FF) is calculated by dividing the maximum power point by open circuit voltage, and short circuit current. The fill factor (FF) of a polymer solar cell is an indicator of the ideality of the diode, although quantitatively, the FF is not a direct percentage of "diodeness." A resistor with no diode characteristics exhibits a FF of $25 \%$. This means that while larger FFs are better, the FF of an illuminated OPV is limited between 25 and 100\%. The ideality of the diode can include anything from the quality of the bulk heterojunction to charge extraction within the device As mentioned before, OPV devices are inherently complex, which complicates the interpretation of OPV electrical characteristics. The FF itself, is a combination of multiple electrical characteristics. Combining these two facts creates a condition that, while the FF data is useful in describing the ideality of the diode, the effects which create that ideality are shielded through complexity. This means that the FF data in this analysis will not be used as rationale for specific mechanisms driving performance, but rather as further evidence in their favor. The data collected for fill factor contained two outliers which were removed 
for analysis (Figure 32).

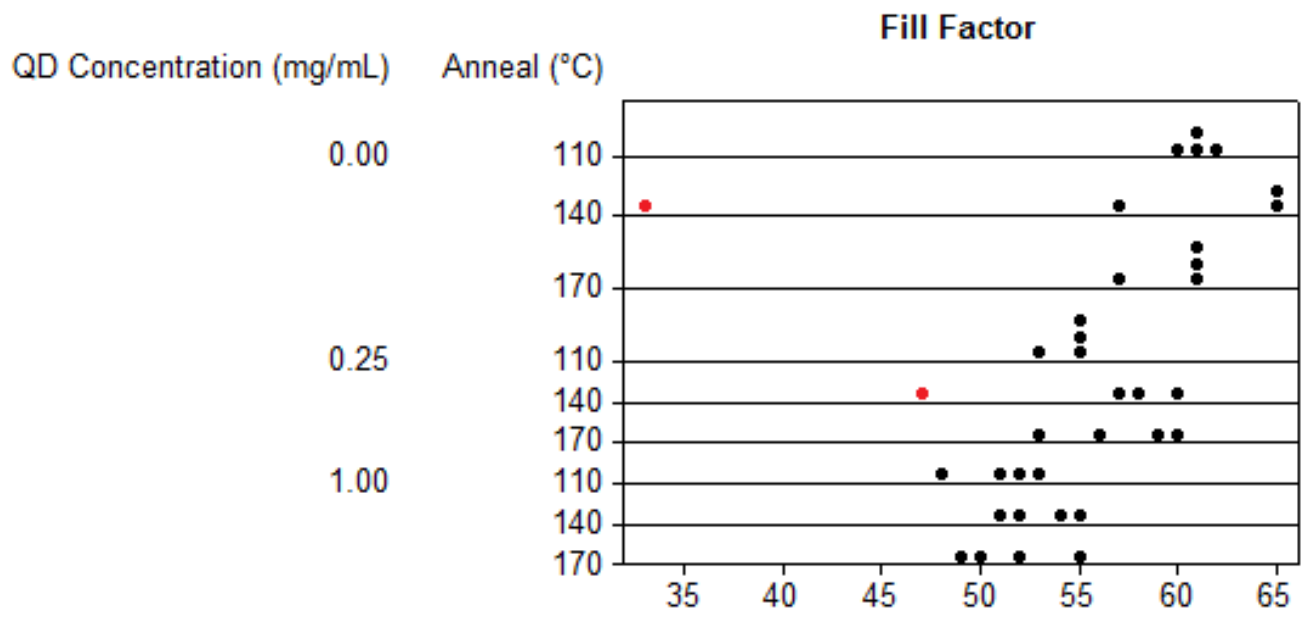

Figure 32: Dot plot analysis for the fill factor response, showing the location of two outliers.

The interaction of QD concentration and anneal temperature was found to be statistically insignificant for the FF, so the analysis was repeated without the interaction in the model. This practice cannot be extended to the pixels, even though their effect was found to be statistically insignificant, because the pixels are an integral part of the data taking procedure. The vast majority of the observed variation was attributed to the addition of quantum dots to the active layer of the device, with pixel effects indistinguishable from random fluctuation (Table V, Figure 33).

Table V: Test statistics and P-Values for Fill Factor

\begin{tabular}{lcc}
\hline \multicolumn{1}{c}{ Effect $\left(\mathbf{R}^{2}=\mathbf{8 2 . 6 3 \%}\right)$} & F-Statistic & P-Value \\
\hline QD Concentration $(\mathbf{m g} / \mathbf{m L})$ & 55.78 & 0.000 \\
Anneal Temperature $\left({ }^{\circ} \mathbf{C}\right)$ & 3.62 & 0.041 \\
Pixel & 2.48 & 0.083 \\
\hline
\end{tabular}




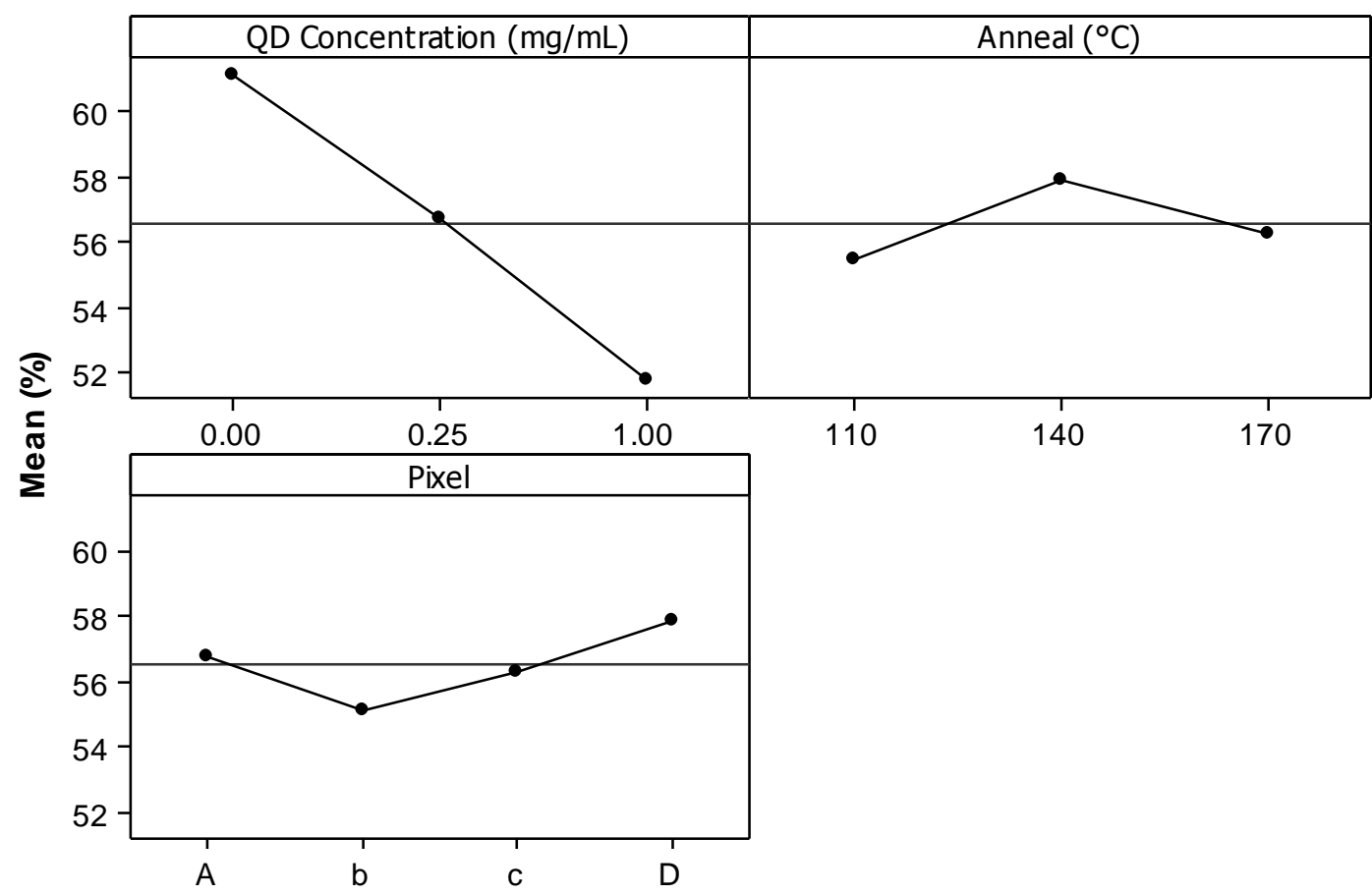

Figure 33: Main effects on the mean of fill factor with the dominating factor of QD concentration.

\section{Power Conversion Efficiency}

The power conversion efficiency (PCE) of a solar cell is a ratio between light incident to the solar cell's surface and electrical power produced by the solar cell. For most practical applications of solar cells, the efficiency of the solar cell predicts how well the solar cell will work. The data collected for PCE showed two underperforming outliers and one over performing data point in the control group annealed at $110^{\circ} \mathrm{C}$. The two underperforming data points were removed on the basis of solar cell degradation as a confounding factor; however the over performing data point cannot be written off as easily. Therefore, for the purposes of this analysis only the two underperforming data points were removed as outliers (Figure 34). 


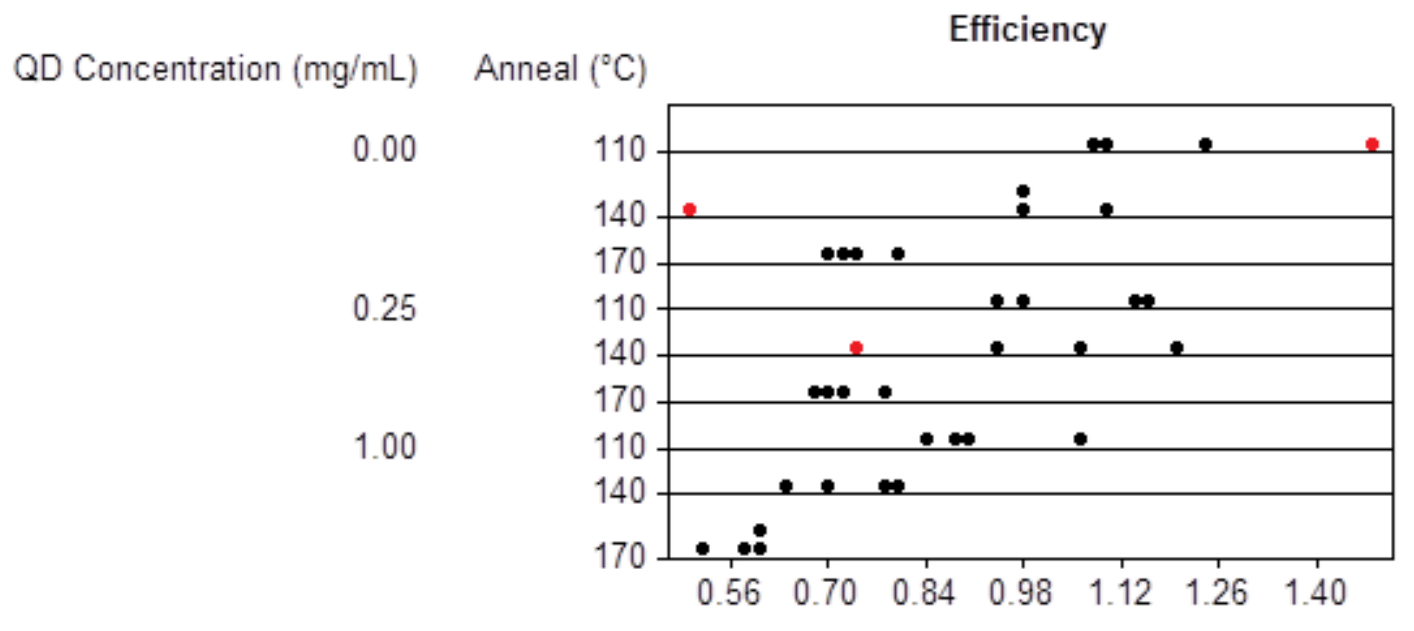

Figure 34: Dot-plot of power conversion efficiency showing the removed outliers in red.

The strongest indicator of OPV power conversion efficiency (PCE) was the anneal temperature (Table VI). This result indicates that the bounds of the anneal input variables were set at or beyond the optimal anneal temperature for OPVs regardless of QD content. The next strongest indicator of PCE was the QD concentration.

Table VI: Test statistic and P-Values for Power Conversion Efficiency.

\begin{tabular}{lcc}
\hline \multicolumn{1}{c}{ Effect $\left(\mathbf{R}^{2}=\mathbf{9 0 . 6 2 \%}\right)$} & F-Statistic & P-Value \\
\hline QD Concentration $(\mathbf{m g} / \mathbf{m L})$ & 53.62 & 0.000 \\
Anneal Temperature $\left({ }^{\circ} \mathbf{C}\right)$ & 118.00 & 0.000 \\
Pixel & 12.80 & 0.000 \\
$\begin{array}{l}\text { Interaction of QD Concentration } \\
\text { and Anneal Temperature }\end{array}$ & 4.09 & 0.013 \\
\hline
\end{tabular}

It is important to remember that the power conversion efficiency was normalized to area. This calls into question why a statistically significant pixel effect was observed. The best explanation arises from the testing procedure; the light source was not moved while the data is taken. The light source is centrally located to the pixels and it is assumed that each pixel receives the same illumination. Another explanation is that the area error discussed earlier affected this data. Although the small pixels underperform compared to the large 
pixels leading to the conclusion that the area error was to blame, the small pixels are located further from the center of the substrate, which supports an error in illumination. Nonetheless, the main effects on the mean reveal that anneal temperatures beyond $110{ }^{\circ} \mathrm{C}$ and the addition of QDs decrease the power conversion efficiency; however, pairwise testing revealed no statistical difference between devices containing $0.25 \mathrm{mg} / \mathrm{mL}$ QDs and devices lacking QDs (Figure 35).

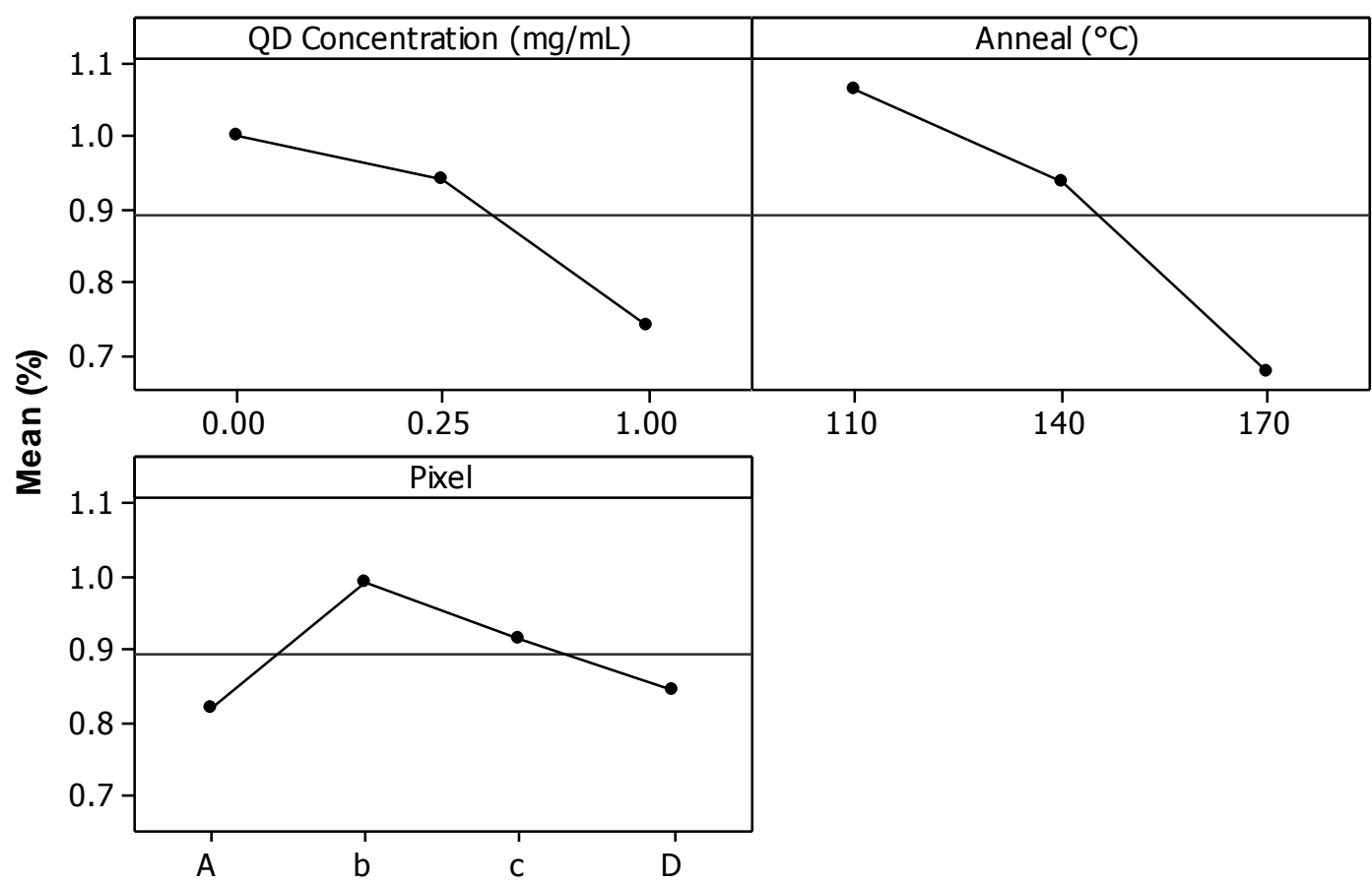

Figure 35: Main effects on efficiency showing small pixels outperforming large pixels and small loading of QDs having little negative effect on the efficiency.

The interactions between QD concentration and anneal temperature are statistically significant; however, since they fall below the extent of pixel interactions, they are overshadowed by confounding factors. 


\section{Series Resistance}

The series resistance of a solar cell represents the difficulty of extracting free charge carriers from the system. The data contained an uncharacteristically large outlier (Figure 36).

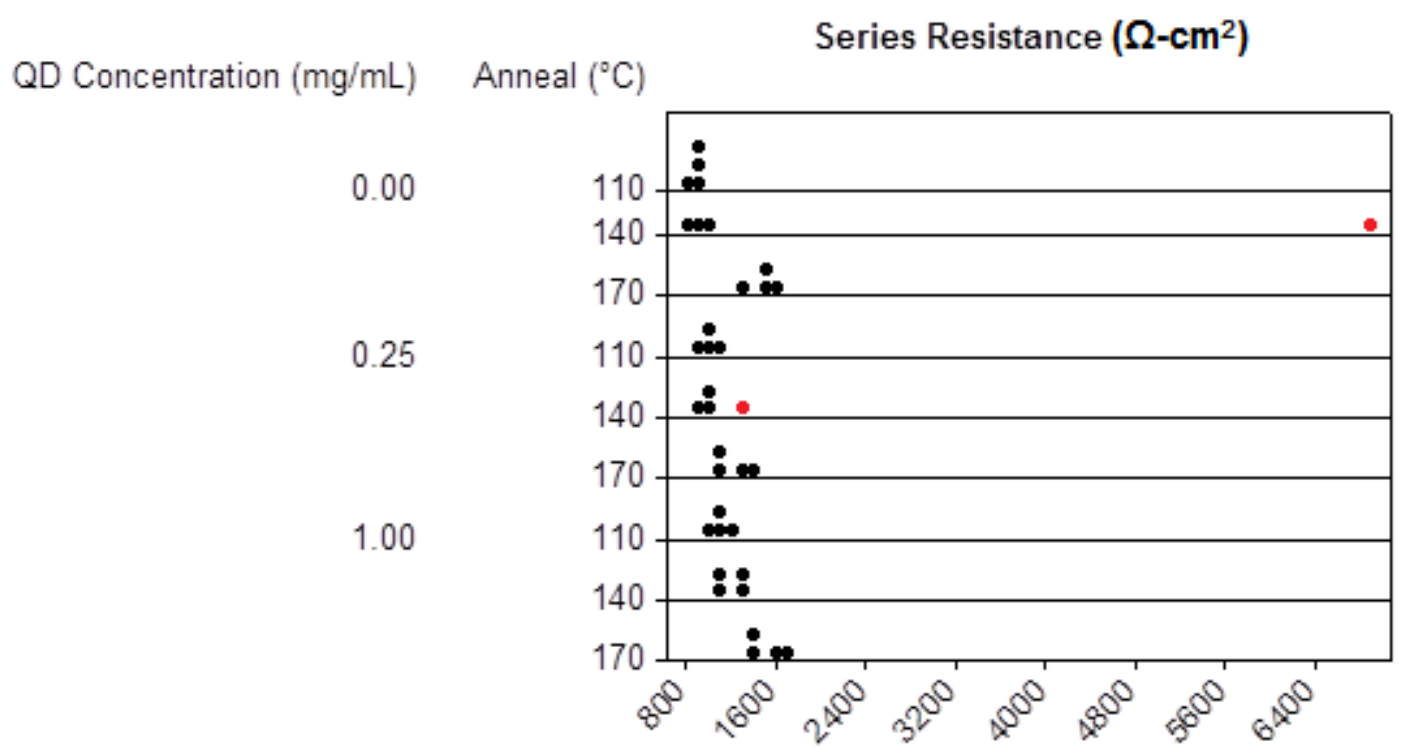

Figure 36: Dot-plot of the response variable, series resistance, showing both outliers in red.

Given that the pixel in the control group annealed at $140^{\circ} \mathrm{C}$ was an outlier in the response of FF, $\mathrm{V}_{\mathrm{OC}}$, and PCE, it is likely that this is an example of a "dead" pixel. When the pixel was omitted from the dot-plot, the typical variation per treatment was observable and another outlier was found (Figure 37). 


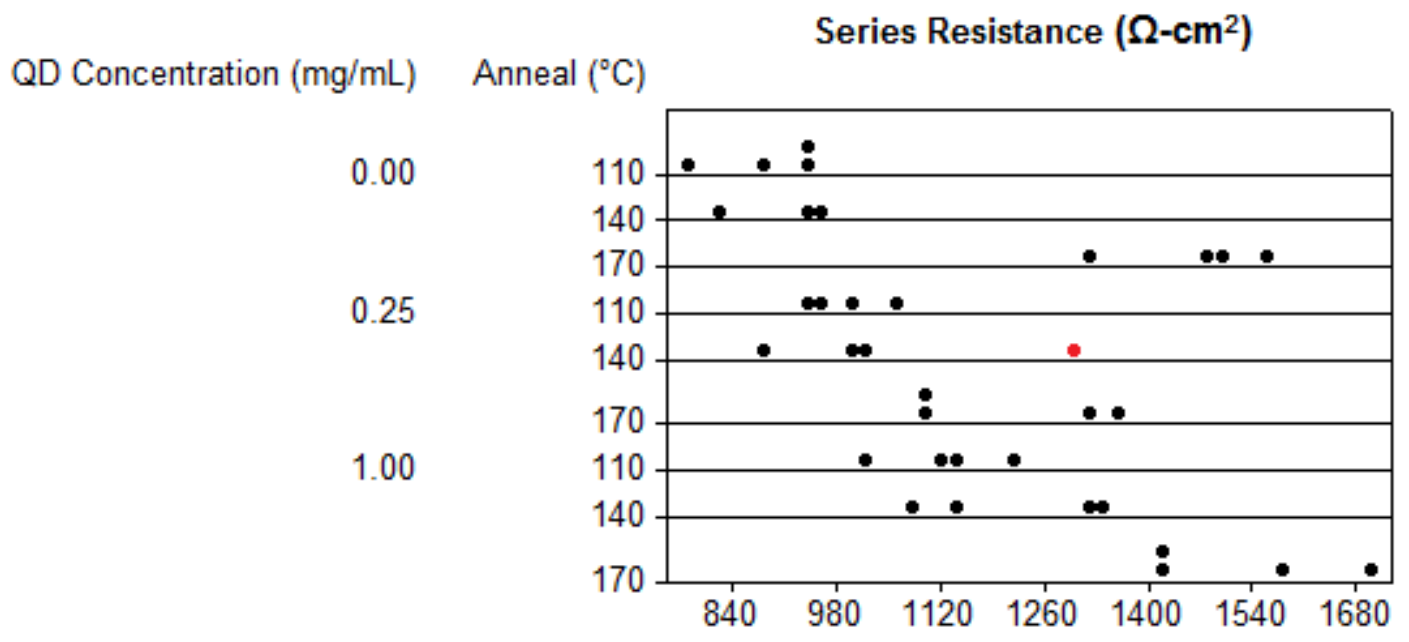

Figure 37: Detail dot-plot of the response variable, series resistance, showing the relative variance between treatments.

No statistically significant interaction between QD loading and anneal temperature was found, allowing for the analysis to be repeated with greater statistical power. In this case, a larger series resistance results in decreased performance. The largest factor showing increasing series resistance was anneal temperature, with the majority of the increase in the $170^{\circ} \mathrm{C}$ anneal condition. QD loading exhibited a smaller effect on series resistance with nearly all of the variability accounted for in the $1.0 \mathrm{mg} / \mathrm{mL}$ QD loading level, while the $0.25 \mathrm{mg} / \mathrm{mL}$ QD loading level was not statistically significant from devices without QDs (Table VII, Figure 38).

Table VII: Test statistics and P-Values for Series Resistance

\begin{tabular}{lcc}
\hline \multicolumn{1}{c}{ Effect $\left(\mathbf{R}^{2}=\mathbf{8 4 . 1 3 \%}\right)$} & F-Statistic & P-Value \\
\hline QD Concentration $(\mathbf{m g} / \mathbf{m L})$ & 15.30 & 0.000 \\
Anneal Temperature $\left({ }^{\circ} \mathbf{C}\right)$ & 49.65 & 0.000 \\
Pixel & 3.24 & 0.038 \\
\hline
\end{tabular}




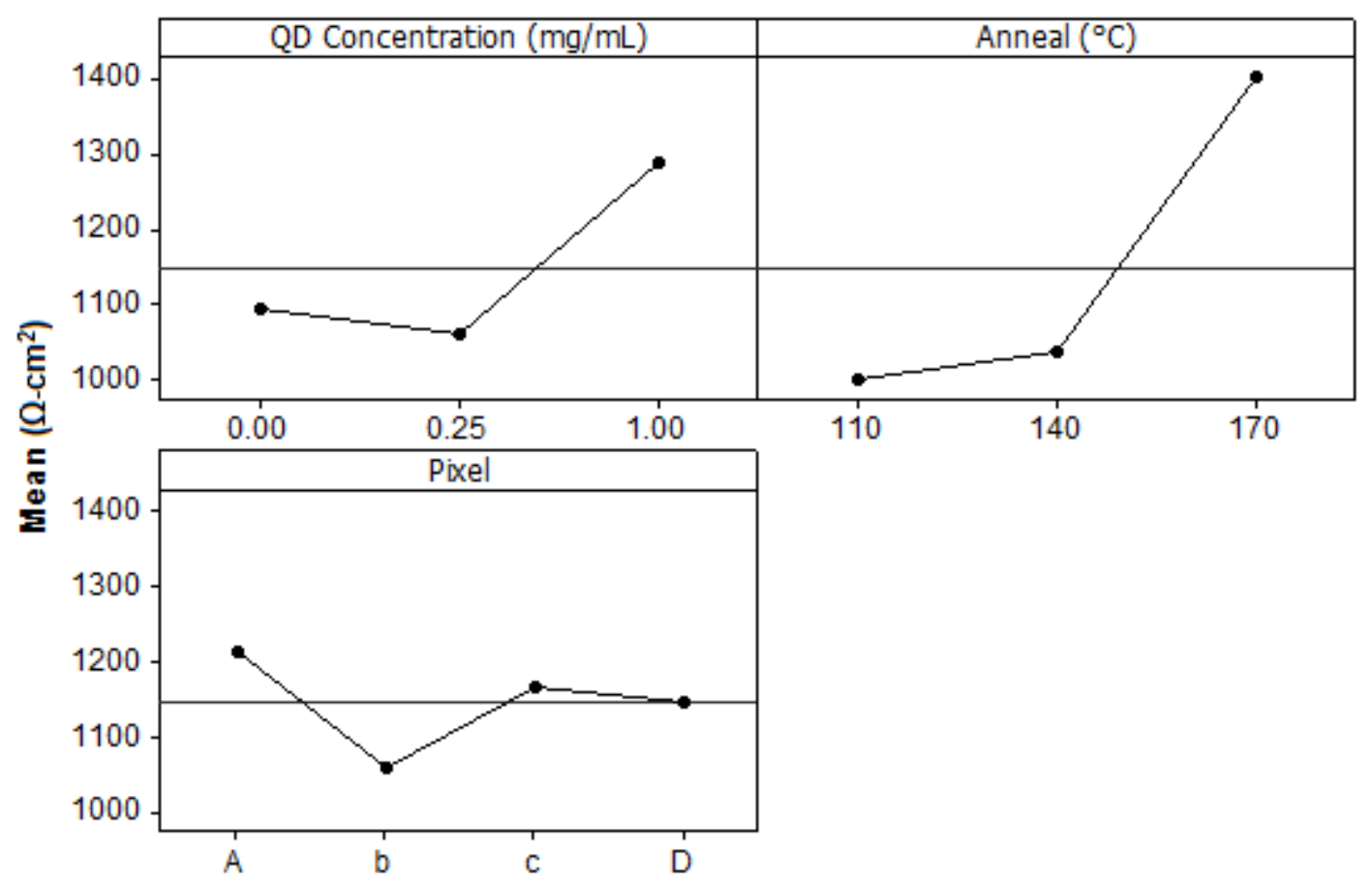

Figure 38: Main effects on the mean of series resistance.

\section{Absorption Characteristics}

All the spectra were taken in a random order to minimize the effect of uncontrollable factors on the data; however, the spectra were normalized to the absorption peak of PCPDTBT for qualitative comparison. Each active layer solution was created by diluting a slightly concentrated stock solution in solvent laden with QDs, which means that each active layer should have equal amounts of the low band gap polymer PCPDTBT. The absorbance peaks of P3HT are highly dependent on the degree of crystallinity and crystallite size while the QDs used have an absorbance peak at $560 \pm$ $10 \mathrm{~nm}$ with a fluorescence peak at $577 \mathrm{~nm}$. The PCPDTBT peak is a broad peak near 740 nm, well outside the range of the expected response, so this was the peak chosen for the normalization. Once the spectra were normalized, each treatment's responses were 
averaged to simplify comparison between anneal treatments and QD loadings. For clarity it is important to note that the anneal treatments are not subsequent to each other. Each substrate underwent a singular anneal treatment for ten minutes, alone and in a random order.

\section{Comparison Between Anneal Temperatures}

Since the analysis of absorption characteristics remains qualitative, the perspective in which the data is presented has an effect on the conclusions that can be drawn. The first perspective, intuitively follows the method that the experiments were conducted and involves the comparison of the QD loading groups within a single anneal treatment. These graphs illustrate which group performs the best in each of the three anneal temperature condition as well as the as-cast condition. 
The as-cast absorbance spectra show the largest loading of QDs with the highest absorption between 450 and $600 \mathrm{~nm}$, with the devices containing no QDs with the lowest and the remainder of the devices between them (Figure 39).

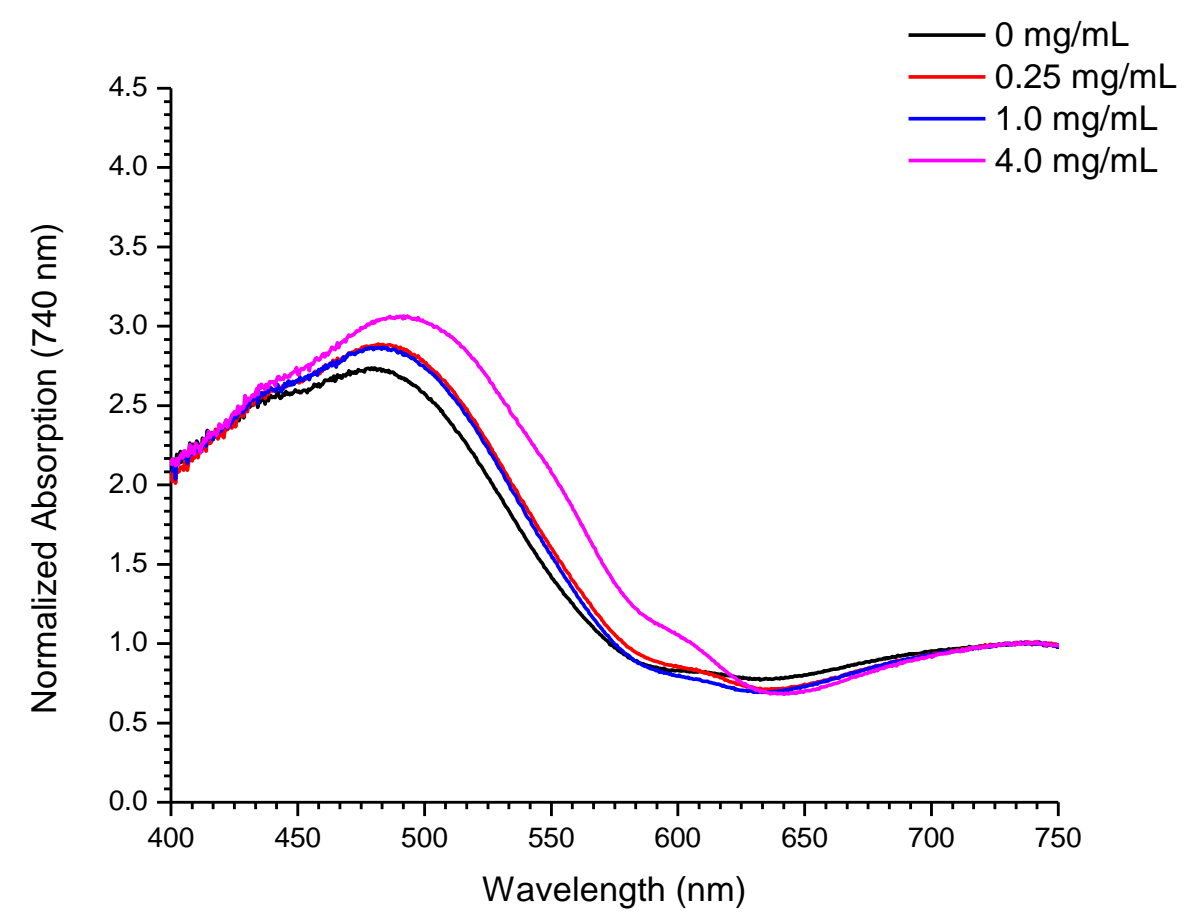

Figure 39: Average normalized absorption spectra before devices are annealed. Each line represents the average of six distinct spectra.

The absorbance of the $0.25 \mathrm{mg} / \mathrm{mL}$ devices and the $1.0 \mathrm{mg} / \mathrm{mL}$ devices seem to be identical, with the two curves splitting after the $560 \mathrm{~nm}$ mark. This highlights the interplay between crystallization and QD loading when absorbance is concerned. The presence of slight bumps, or "shoulders," near $550 \mathrm{~nm}$ and $600 \mathrm{~nm}$ are indicative of the crystallinity of P3HT. Since these shoulders are present on the spectra containing QDs and absent on the spectra lacking QDs and the spectra were taken before annealing, it is likely that the QDs serve to aid crystallite formation, perhaps as nucleation sites. 
The general practice for these devices states a ten minute anneal at $110{ }^{\circ} \mathrm{C}$ is sufficient to provide optimal growth of the BHJ. The absorbance spectra of the QD groups all outperform the control devices between $450 \mathrm{~nm}$ and $620 \mathrm{~nm}$; however, all of the QD device absorbance spectra indicate high levels of crystallinity (Figure 40).

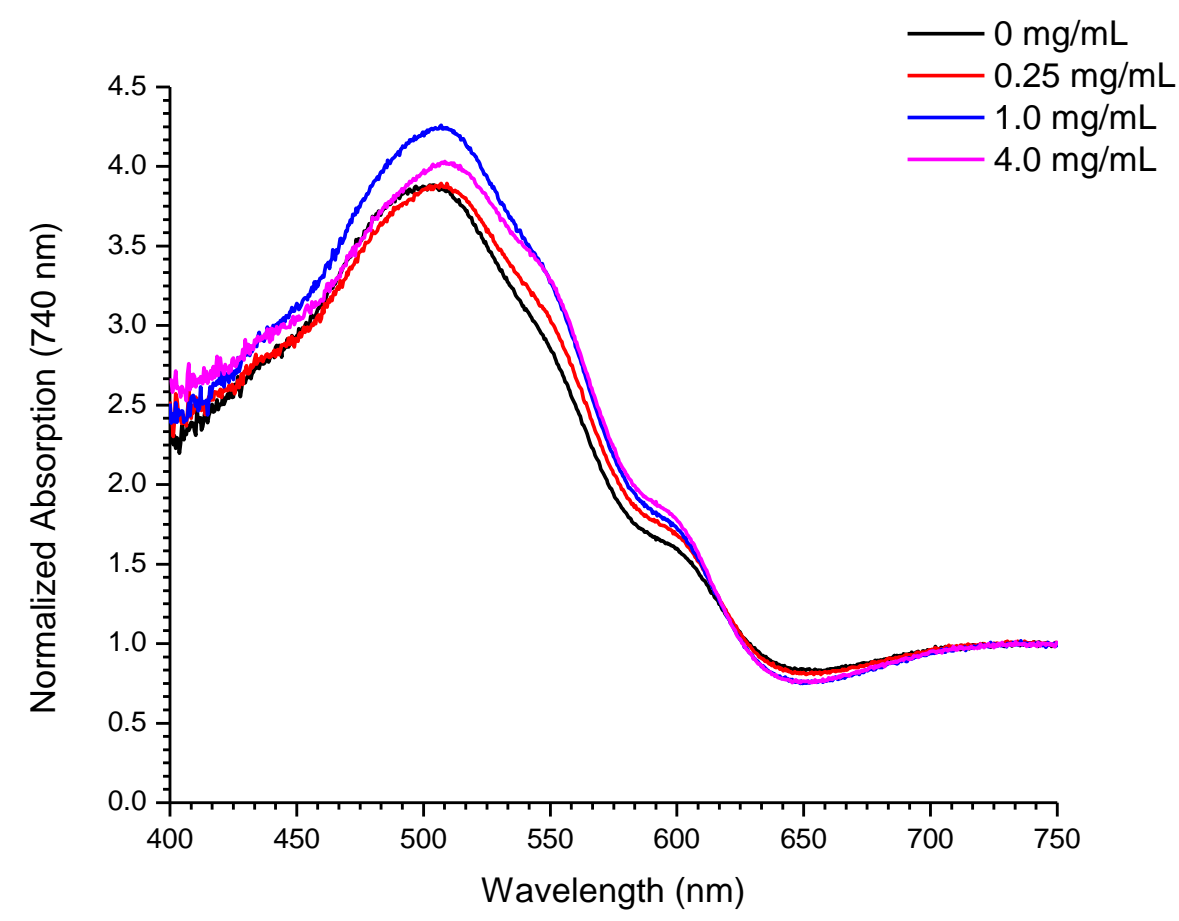

Figure 40: Average normalized absorption spectra after annealing at $110{ }^{\circ} \mathrm{C}$ for ten minutes.

It is worth mentioning that the increased absorbance in the QD laden devices did not lead to favorable electronic characteristics, as mentioned in the previous section. In fact, the only QD group that did not show significant loss of performance at this anneal temperature was the $0.25 \mathrm{mg} / \mathrm{mL}$ QD concentration, which shows a similar amorphous response at $\sim 510 \mathrm{~nm}$ to the group with no QDs. 
When annealed for ten minutes at $140{ }^{\circ} \mathrm{C}$, the lowest level of QD loading exhibited the largest OD at $\sim 510 \mathrm{~nm}$ compared to the highest level of QD loading (Figure 41).

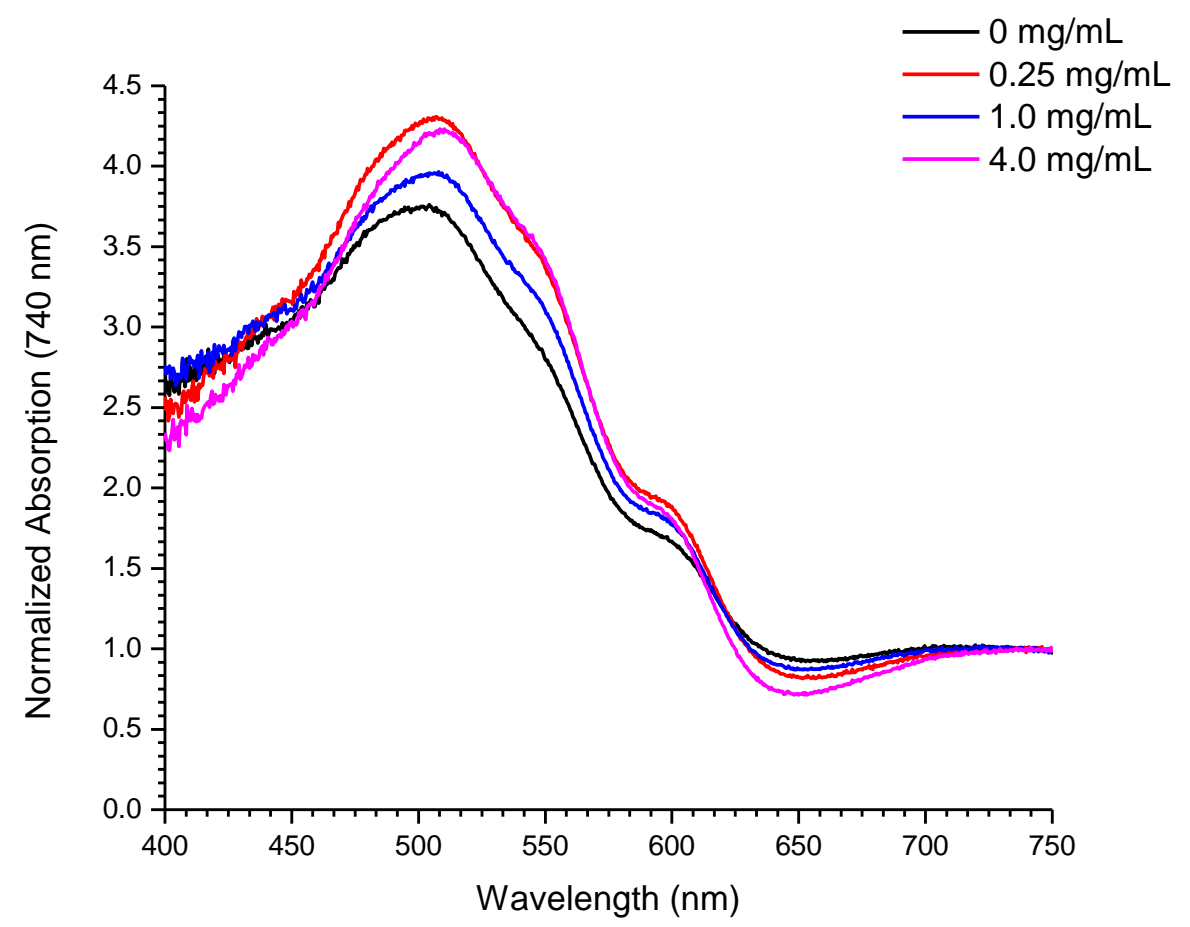

Figure 41: Average normalized absorption spectra after annealing at $140{ }^{\circ} \mathrm{C}$ for ten minutes.

In fact, the lowest level of QD loading showed a greater increase than when annealed at $110^{\circ} \mathrm{C}$ in its overall absorptivity while both the middle loading of QDs and the devices without QDs showed smaller increases in overall absorptivity than when annealed at the lower temperature. While the highest and lowest level of QD loading showed similar absorbance characteristics, electronically they were the furthest apart. The devices without QDs and the lowest QD loading level were electrically similar under this anneal condition, to the point of being statistically insignificant from each other in power conversion efficiency. 
When annealed for ten minutes at $170{ }^{\circ} \mathrm{C}$ the lowest level of QD loading showed the least degradation in absorptivity, while all other groups showed strong evidence of decreased spectral response when compared to any of the lower anneal temperatures (Figure 42).

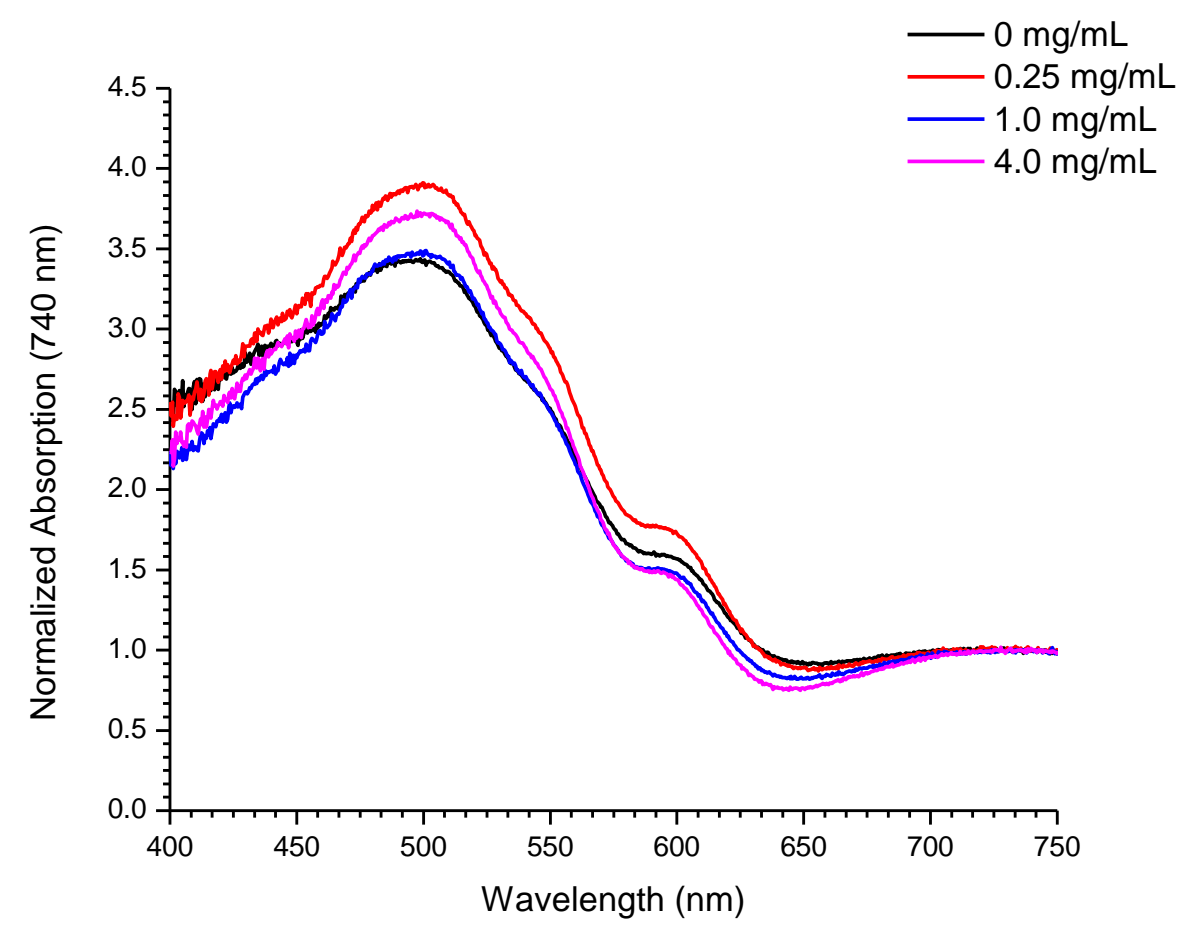

Figure 42: Average normalized absorption spectra after annealing at $170{ }^{\circ} \mathrm{C}$ for ten minutes.

The electrical characteristics of all devices under the $170{ }^{\circ} \mathrm{C}$ anneal condition were poor.

Given the poor electrical performance, it is safe to assume that the absorption

characteristics of these devices could offer insight into the morphology driving the poor electrical performance.

\section{Comparison of Quantum Dot Loading Over Anneal Temperatures}

By grouping the spectra together by QD loading the effects of annealing the devices becomes more clear. In these graphs, the absorption spectra for a single group evolves over anneal conditions. This perspective allows a typical model to be set up with the 
devices that lack QDs, which serves as our qualitative "control” that each QD loading level is compared with.

In general, these devices exhibit a large gain in absorbance between the as-cast state and a ten-minute anneal at $110{ }^{\circ} \mathrm{C}$, while annealing at higher temperatures results in degraded peak absorption and higher degrees of P3HT crystallinity (Figure 43).

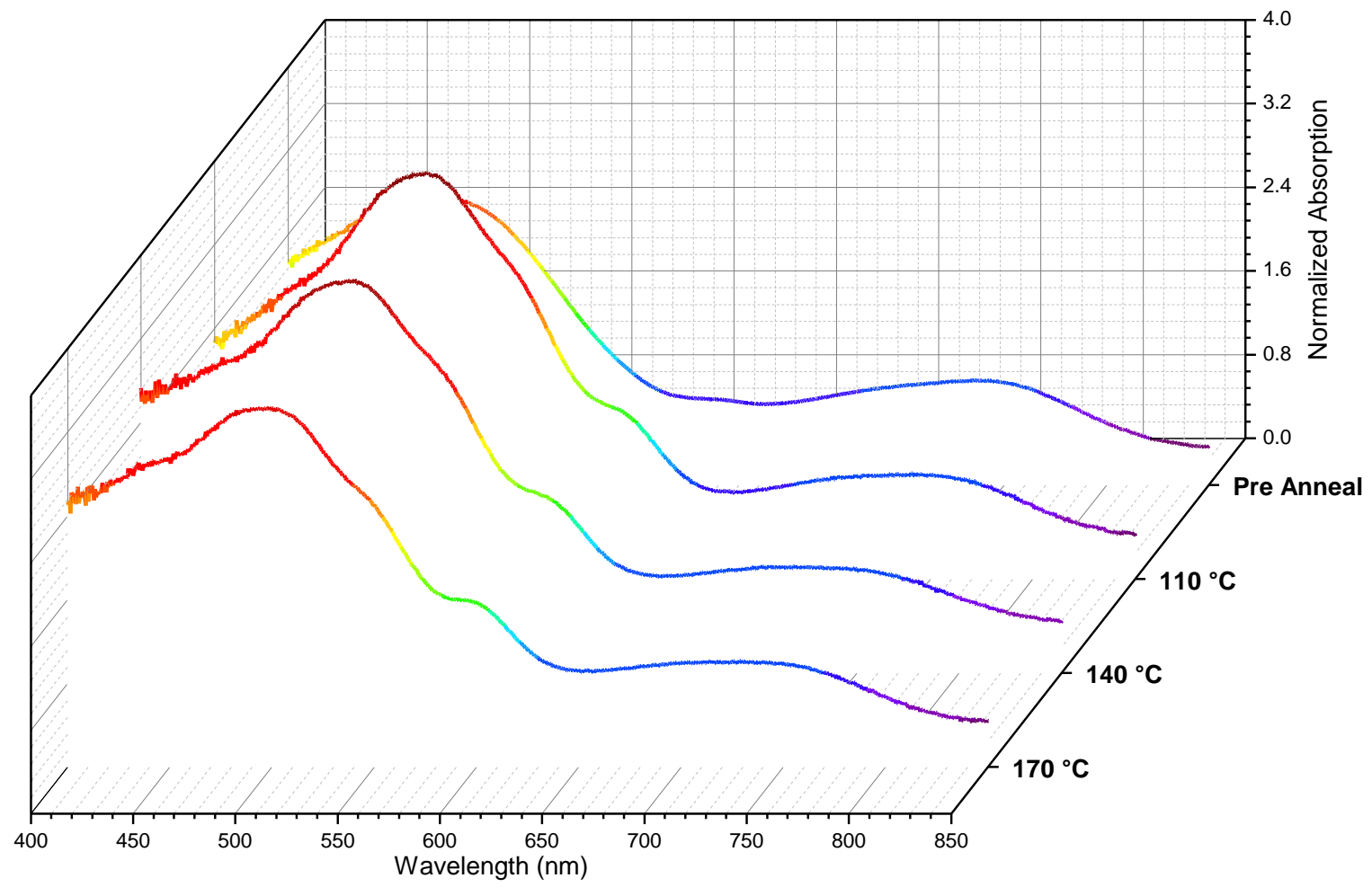

Figure 43: Average absorption spectra of devices without QDs showing a typical diffuse absorption before annealing and increasingly crystalline "shoulders" as anneal temperature increases. The color of the line denotes the value along the normalized absorption spectra.

These devices showed a maximum normalized absorption of nearly four, meaning that the maximum absorption was four times greater than the absorption response of the PCPDTBT "peak" at $740 \mathrm{~nm}$. Due to the complex nature of the graph, the color of the line illustrates value on the Normalized Absorption axis with dark red as the highest value and a deep purple as the lowest value with the normal color spectrum in between. 
Adding a small amount of QDs to the active layer increases the initial degree of crystallization; however, a higher temperature anneal is required to maximize the spectral response. Once the spectral response was maximized, this loading group showed the typical degradation in maximum peak size and increasing "shoulder" intensity (Figure 44).

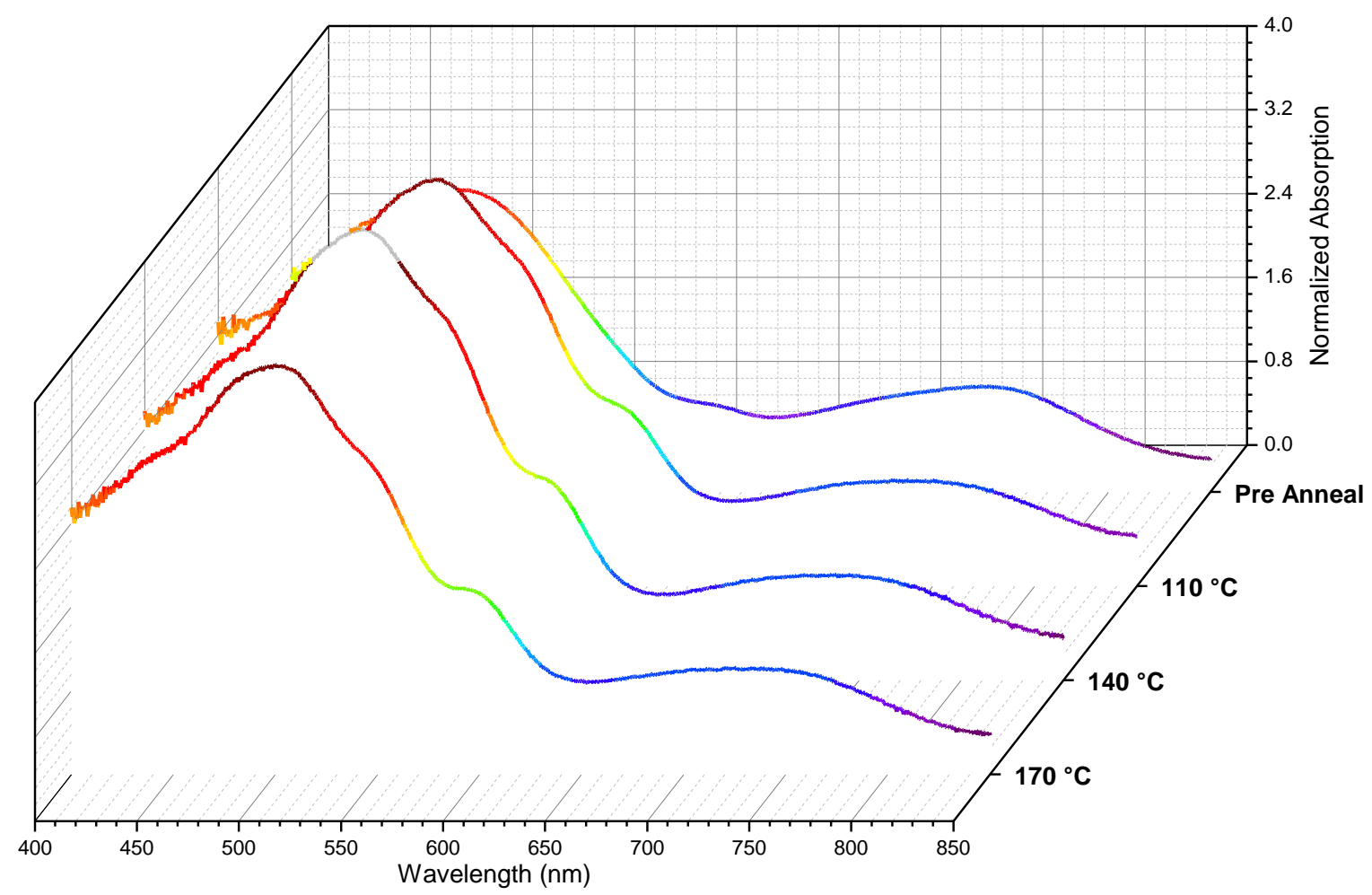

Figure 44: Average absorption of devices containing $0.7 \mathrm{wt} \%$ QDs before annealing and with each separate anneal treatment. The color of the line denotes the value along the normalized absorption spectra and the grey indicates off-scale absorption with the scale limited to the absorption of devices without QDs.

The color of the line turned to grey as the line went off the scale on the Normalized Absorption axis. This illustrates the treatments that have a greater spectral response than the best treatment for devices without QDs. 
The medium QD loading level shows a weaker increase in crystallinity in the preanneal state, and its spectral response was maximized with the $110^{\circ} \mathrm{C}$ anneal (Figure 45).

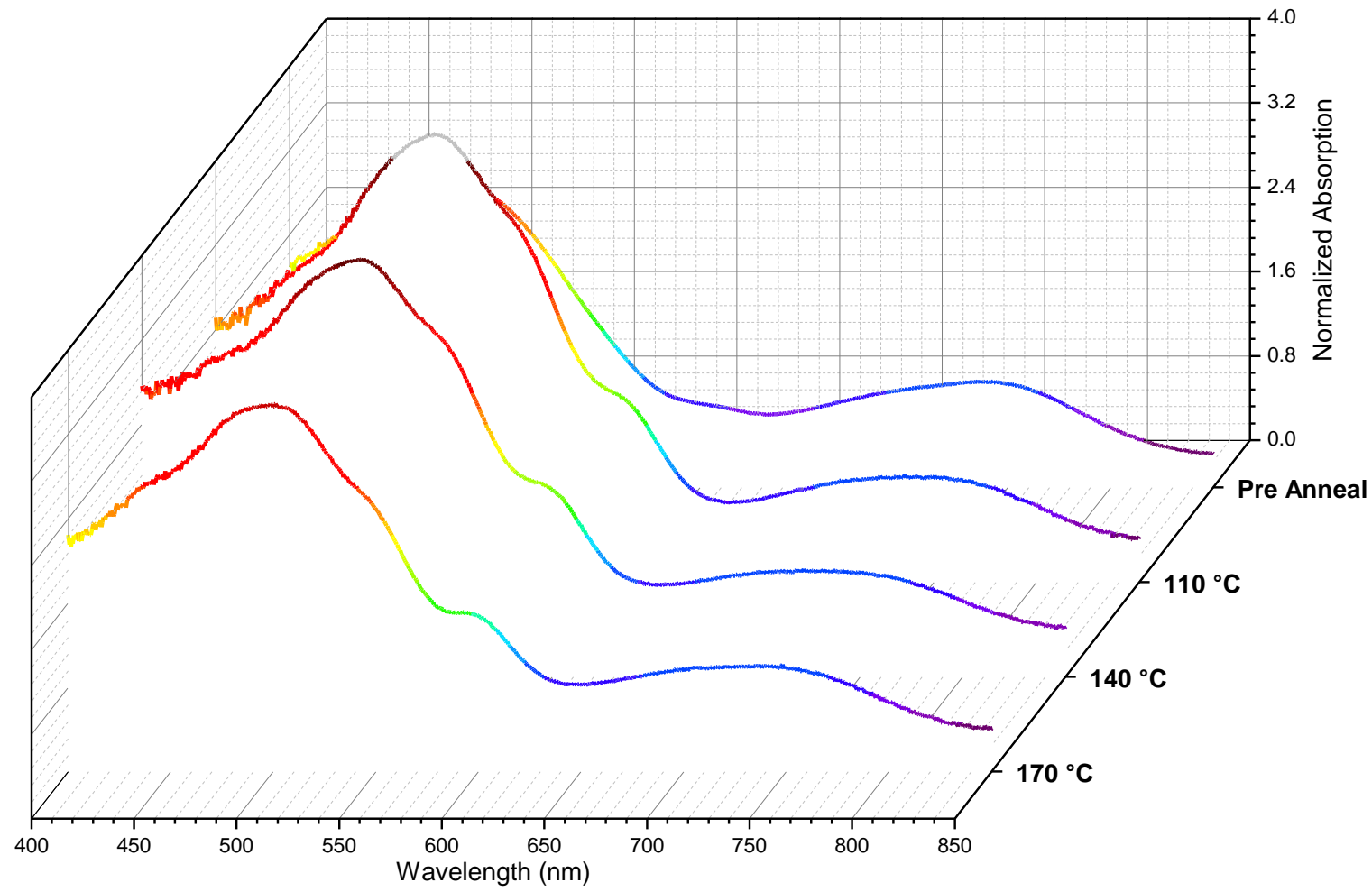

Figure 45: Average absorptivity spectra of devices containing $2.7 \mathrm{wt} \%$ QDs, showing a maximized absorption response at the $110^{\circ} \mathrm{C}$ anneal.

This result was not intuitive, as the other two QD loadings were both maximized at the $140^{\circ} \mathrm{C}$ anneal. This suggests a secondary mechanism was at work between the QD groups that begins to dominate as concentration increases. The secondary mechanism must also interfere with the increase of absorption through more beneficially ordered crystalline and amorphous P3HT regions.

The highest QD loading level exhibited large degrees of initial crystallization with a maximized spectral response at the $140{ }^{\circ} \mathrm{C}$ anneal (Figure 46). 


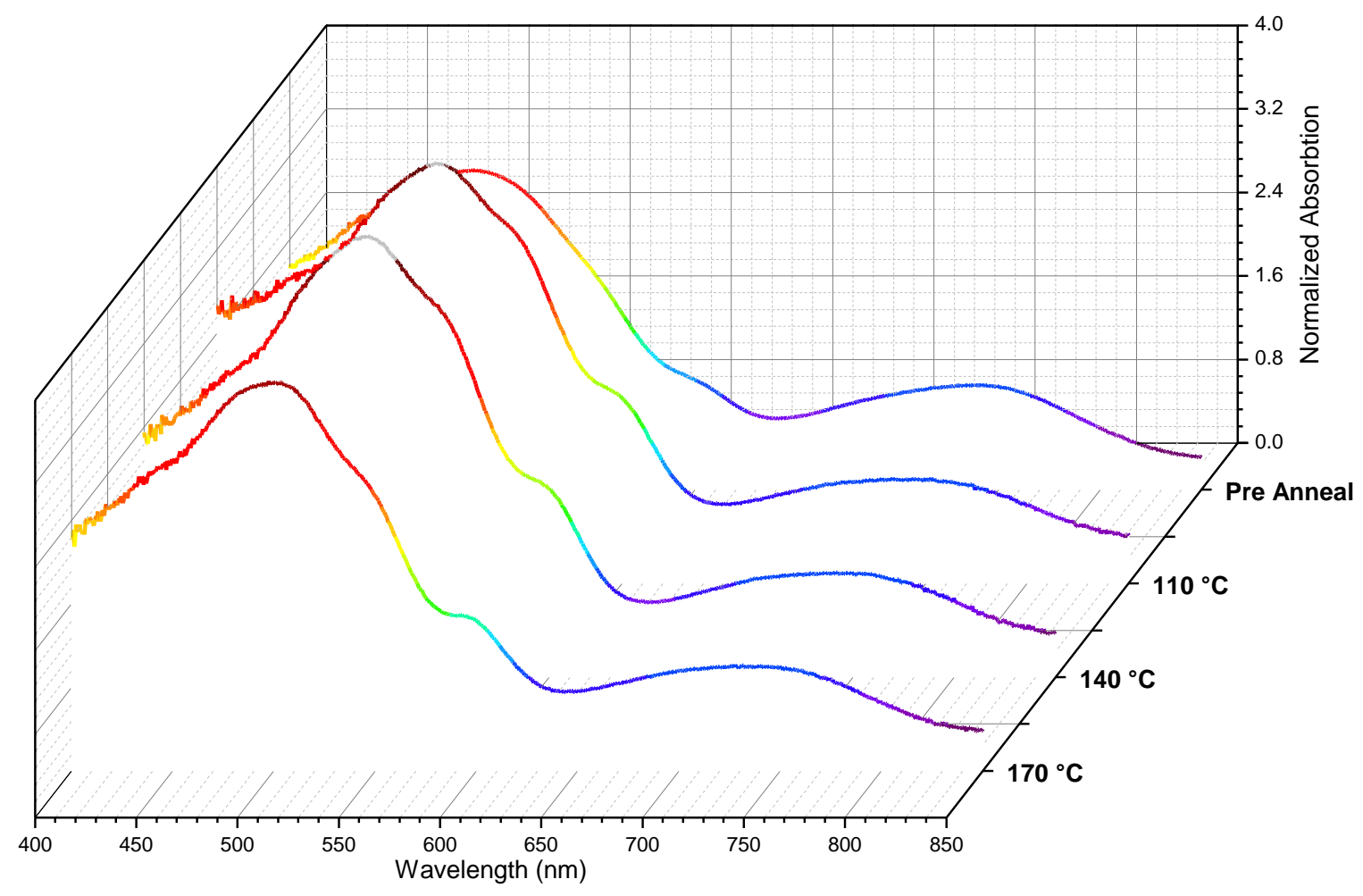

Figure 46: Average absorption of devices containing $10.0 \mathrm{wt} \%$ QDs. 


\section{As-Cast and Post Anneal Comparison}

Considering the best performance that the OPVs can achieve gives a picture of the efficacy of the technology; however, the best performance of a technology is not the only metric that should be considered. Viewing the extent of change between initial performance and final performance can give insight into the mechanisms behind that change. In P3HT:PCBM OPVs, annealing the as-cast films results in huge performance increases. These performance increases are attributed to morphological changes to the BHJ. It follows that QD laden OPV devices that underperform when compared to PCPDTBT-P3HT:PCBM devices, would show changes in the percentage difference between as-cast and annealed conditions that would allow insight into their poor performance.

The percentage difference is a measure of change from a reference set in terms of the reference. In this case it is the annealed response minus the as-cast response, divided by the as-cast response. Since the QD laden groups exhibited poor performance after annealing, it is safe to assume the effects of anneal outweigh the initial conditions of the film, that is to say that annealing the devices provides a better route to device performance increases than adding QDs; however, the as-cast condition adds to the fullness of the discussion.

The as-cast OPV films exhibit large variation within groups because the rate of defects within the film is high, as the chlorobenzene solvent evaporates quickly allowing little time for the film to relax into a more ordered form. In PCPDTBT-P3HT:PCBM devices without QDs, this results in higher $\mathrm{V}_{\mathrm{OC}}$, lower $\mathrm{J}_{\mathrm{SC}}$, and larger series resistance. Once annealed, the $\mathrm{V}_{\mathrm{OC}}$ and series resistance drop while the $\mathrm{J}_{\mathrm{SC}}$ increases dramatically, 
all of which contributes to higher fill factors and power conversion efficiency. In comparison, devices containing QDs exhibit an initially higher $\mathrm{J}_{\mathrm{SC}}$, lower series resistance, lower shunt resistance, lower fill factors, and higher efficiency. Again, the effects observed from annealing far exceed any initial difference. So while the initial condition could influence a percentage difference analysis, that effect in this study is in the same order of magnitude, or smaller than, pixel effects. The overall effect of QD loading on as-cast quality of the films, and change due to annealing was complex, but each effect led to poorer performance in devices containing QDs (Table VIII).

Table VIII: Quantum Dot Sensitized Cells Compared to the PCPDTBTP3HT:PCBM Controls

\begin{tabular}{|c|c|c|c|c|c|c|}
\hline & $\mathbf{J}_{\mathbf{S C}}$ & $\mathbf{V}_{\text {OC }}$ & PCE & FF & Shunt & Series \\
\hline As-Cast & $\nearrow$ & Same & $\lambda^{1}$ & $\searrow$ & $\searrow$ & $\searrow$ \\
\hline $\begin{array}{l}\Delta \text { Due to } \\
\text { Annealing }\end{array}$ & rLess & $\searrow$ More & rLess & $\begin{array}{c}0.25 \nearrow \\
1.0 \searrow\end{array}$ & $\begin{array}{l}0.25 \nearrow \\
1.0 \searrow\end{array}$ & $\searrow$ Less \\
\hline $\begin{array}{l}\text { Final Device } \\
\text { Performance }\end{array}$ & Same & Lower & Lower & Lower & Same & Higher $^{2}$ \\
\hline $\begin{array}{l}\text { Temperature } \\
\text { Dependence } \\
\text { of } \Delta \text { Due to } \\
\text { Annealing }\end{array}$ & $\searrow^{3}$ & $\searrow$ & $\searrow^{3}$ & $\begin{array}{l}140 \nearrow \\
170 \searrow\end{array}$ & $\nearrow$ & None \\
\hline $\begin{array}{l}\text { Temperature } \\
\text { Dependence } \\
\text { of Final } \\
\text { Device } \\
\text { Performance }\end{array}$ & $\searrow$ & $\searrow$ & $\searrow$ & Same & Same & $\lambda^{3}$ \\
\hline
\end{tabular}

The short circuit current density, for example, was higher in QD sensitized devices in the as-cast condition. Annealing the devices increased the short circuit current density of all 
the devices, but QD sensitized devices improved less than the control. The final short circuit current density values were the same between the groups. Lastly, the temperature dependence on the magnitude of the change in short circuit current density and the magnitude of the final value were smaller in devices sensitized with QDs.

\section{Short Circuit Current Density}

By far the largest effect on the percentage difference between as-cast and annealed states was the short circuit current density. This large effect was expected, even depended upon, as it is this increase in charge mobility that drives the huge performance increases between as-cast and annealed devices. That being said, annealing beyond $110^{\circ} \mathrm{C}$ was shown as universally bad throughout these results, so performance degradation without QDs should be explained. It is suspected that the suppression of gain in short circuit current in the 140 and $170^{\circ} \mathrm{C}$ anneal condition is due to overly large $\mathrm{BHJ}$ regions with increased recombination current. This degradation effect is based on ability of the BHJ constituents to move in relation to each other in order to from larger, lower energy, single constituent regions. P3HT becomes more mobile allowing PCBM to diffuse out of the P3HT and form small PCBM crystallites, while the newly evacuated P3HT regions align to form highly ordered regions of backbone stacking. This backbone stacking allows charge to transfer more easily between polymer chains, and is the source of the severalfold increase in device performance. The addition of QDs to the devices inhibited the increase of short circuit current nearly three times more than the observed effect of over annealing, suggesting that the QDs themselves are causing the decrease without increasingly large BHJ regions increasing recombination current (Figure 47). 


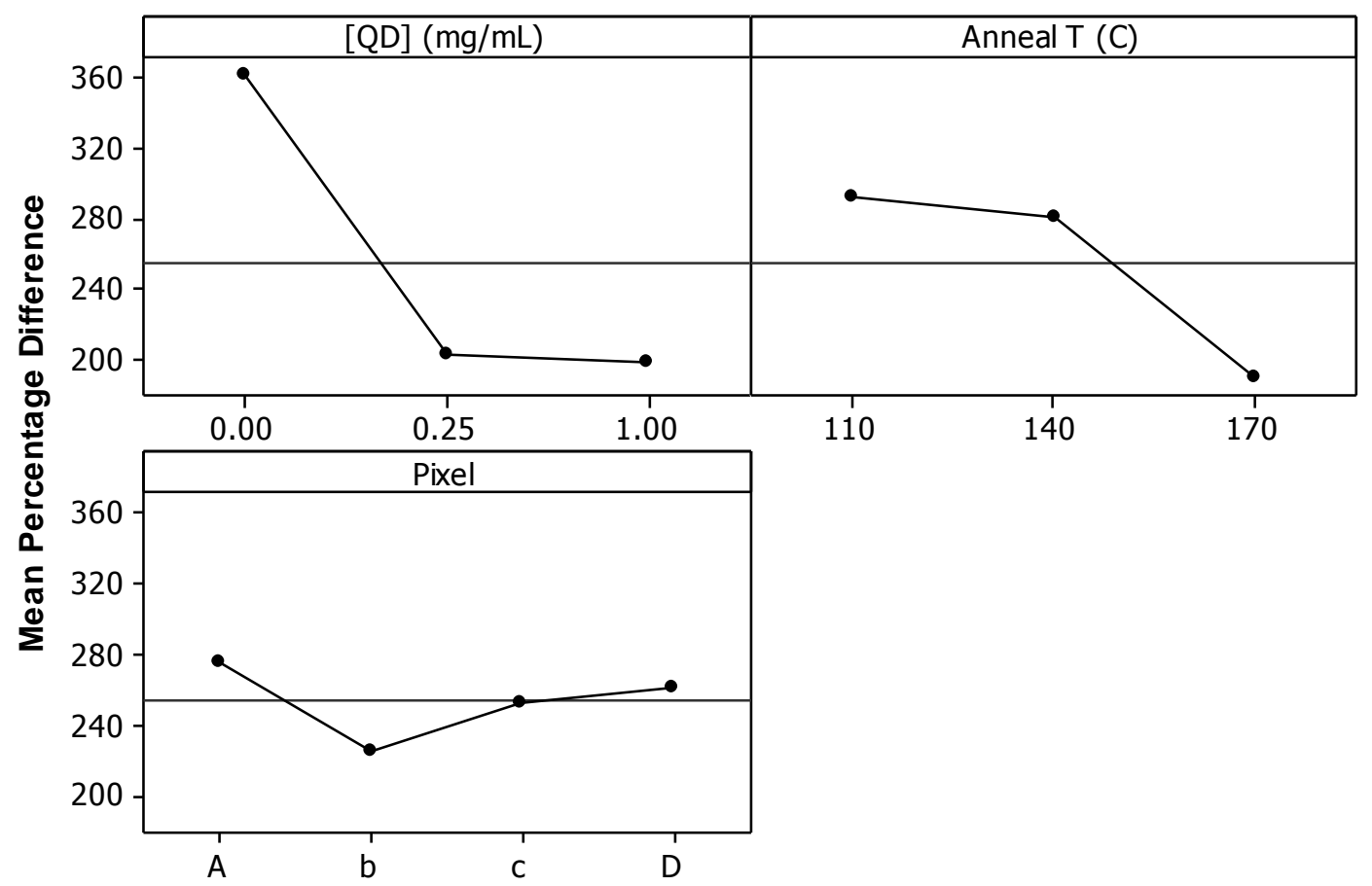

Figure 47: Main effects on the mean percentage difference between as-cast and annealed conditions, showing a large effect due to QDs with a smaller effect based on anneal temperature.

The final $\mathrm{J}_{\mathrm{SC}}$ value exhibited a large temperature dependence with a statistically significant effect with QD loading that did not exhibit a trend. This does not necessarily mean that no effect exists, but rather suggests that an effect exists which is overshadowed by the large temperature dependence.

\section{Open Circuit Voltage}

The as-cast $\mathrm{V}_{\mathrm{OC}}$ of $0.25 \mathrm{mg} / \mathrm{mL}$ and $1.0 \mathrm{mg} / \mathrm{mL}$ was found to be statistically insignificant from the devices without QDs. Each level of QD loading was found to have a statistically significant percentage difference, while only the $110^{\circ} \mathrm{C}$ anneal was statistically different from the other anneal treatments. Both increasing QD loading, and increasing anneal temperature, furthered the decrease in $\mathrm{V}_{\mathrm{OC}}$. The known inputs that affect the $\mathrm{V}_{\mathrm{OC}}$ are LUMO-HOMO offset between acceptor and donor material, LUMO-LUMO offset between donor and acceptor material, and the work function of the top electrode. The 
LUMO-HOMO offset has the strongest effect on $\mathrm{V}_{\mathrm{OC}}$ and acts as the limiting input factor, while the other two effects are based on the excitonic nature of OPVs creating poor charge extraction, decreasing the potential of excited electrons and holes. Since QD loading has roughly three times the effect on $\mathrm{V}_{\mathrm{OC}}$ drop as anneal temperature, the QDs must be contributing to the $\mathrm{V}_{\mathrm{OC}}$ drop in a substantial way. Since a trend was observed in both QD loading level and anneal temperature, any proposed model should explain both. In the control devices, the drop in $\mathrm{V}_{\mathrm{OC}}$ is attributed to growing PCBM rich regions. As the PCBM forms crystallites, the number of excited states available increases, which widens the LUMO level of the acceptor, lowering the LUMO of the acceptor. The higher the temperature of the anneal, the more mobile the system, the larger the PCBM crystallites can grow. A smaller LUMO-HOMO offset decreases the $\mathrm{V}_{\mathrm{OC}}$. Since it is unlikely that this effect would increase with QD loading, and the observed drop in QD groups was much larger than that of the control, another mechanism for $\mathrm{V}_{\mathrm{OC}}$ drop must be present in groups containing QDs which is also temperature dependent (Figure 48). 


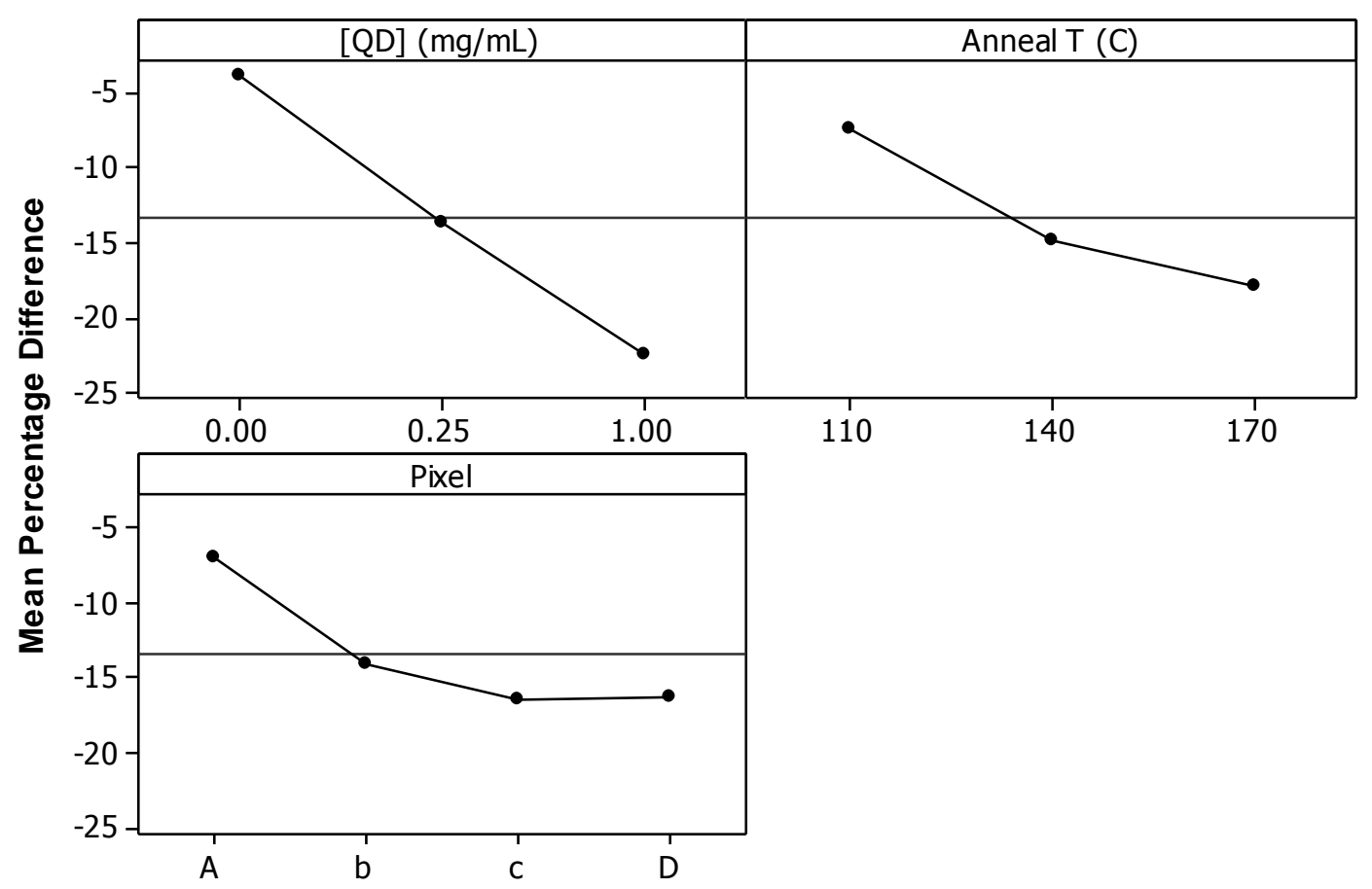

Figure 48: Main effects on the mean percentage difference between as-cast and annealed devices, showing a larger decrease in $\mathrm{V}_{O C}$ due to QD loading than to anneal temperature.

\section{Fill Factor}

The as-cast films showed decreased fill factors for the QD laden devices, and no statistical difference between the two groups. In the percentage difference analysis, the fill factor showed a statistically significant interaction between the QD loadings and annealing temperature. This means that the effect of anneal temperature was different for at least one of the QD loading levels. It is important to note that the QD laden groups both started and ended with lower FFs than the control, and the control group annealed at $110^{\circ} \mathrm{C}$ outperformed the group annealed at $140^{\circ} \mathrm{C}$. Thus, while the interactions observed in the percentage difference analysis give insight into phenomena present during the annealing process for the different devices, it absolutely does not inform a decision about the best processing parameters. 


\section{Series Resistance}

The series resistance decreased less with groups containing QDs, giving evidence of a detrimental morphology of the BHJ components proportional to QD loading (Figure 49).

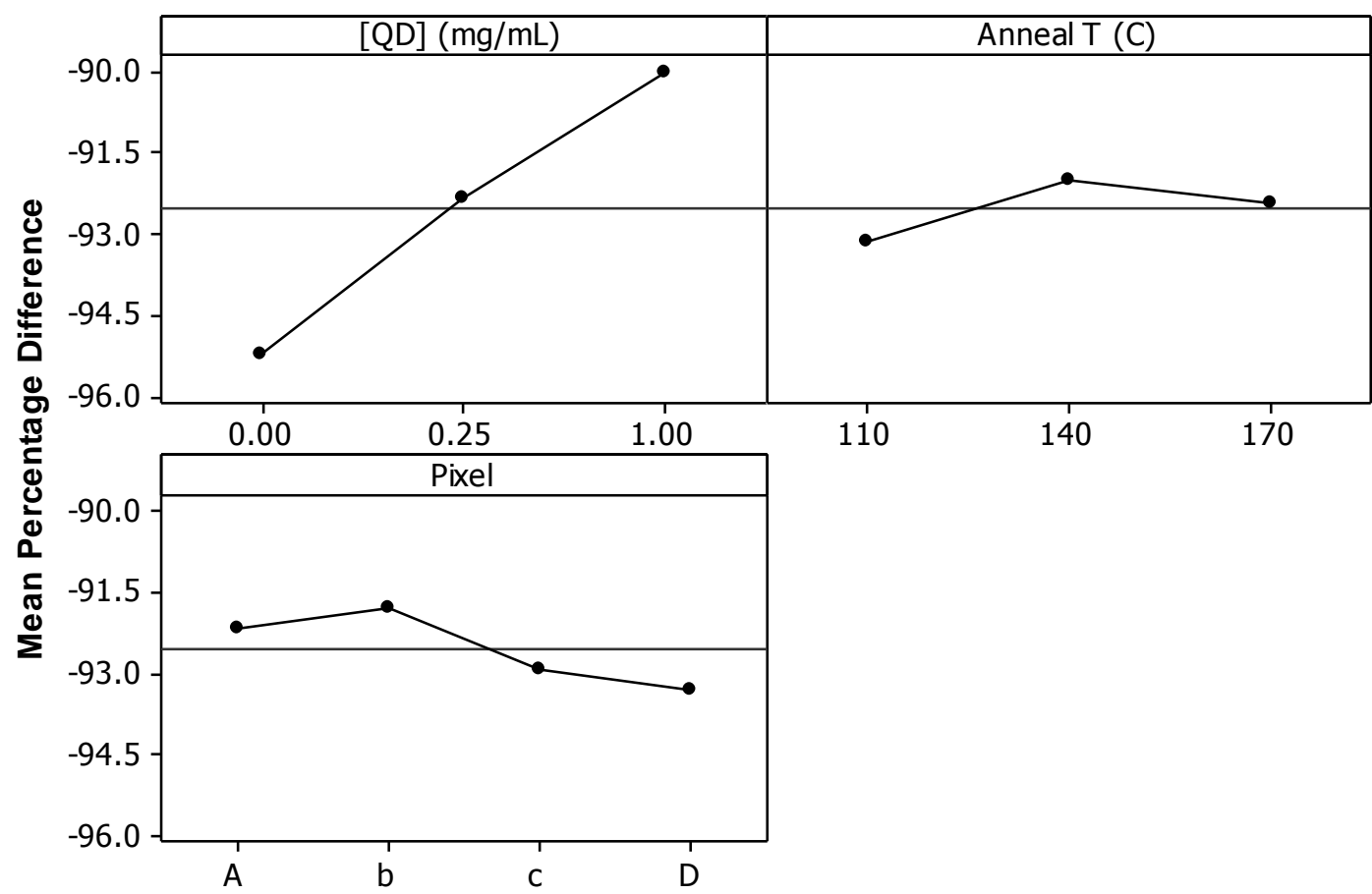

Figure 49: Main effects on the mean percentage difference between annealed and as-cast states' series resistance showing a smaller decrease in series resistance with QD loading and a nuetral response with anneal temperature.

The final series resistance values were higher in groups containing QDs. Higher series resistance values, counter to intuition, do not necessarily create smaller $\mathrm{J}_{\mathrm{SC}}$ values. Instead, they indicate the shape of the J-V curve, and show the origin of a poor FF.

\section{Atomic Force Microscopy and Electrostatic Force Microscopy}

Atomic force microscopy (AFM) has proven to be an incredibly versatile imaging and characterization technique. ${ }^{65}$ Electrostatic force microscopy (EFM), combines the surface imaging of the AFM with an applied voltage. As the AFM tip scans the surface, it records the height of the sample on a point-by-point basis. Once this height distribution is recorded, the bias voltage is applied and the AFM tip retraces the line it previously 
recorded at a set distance away from the surface profile previously recorded. This technique yields traditional height imaging as well as the dynamic response a material has to an applied bias without the influence of height variation. ${ }^{65}$

Since all of this is data is recorded from an oscillating tip, the data is in essence the disturbance of the AFM tip vibration. The disturbance in amplitude yields the surface imaging, and the disturbance in phase gives phase imaging, which is an altogether different kind of image (Figure 50).
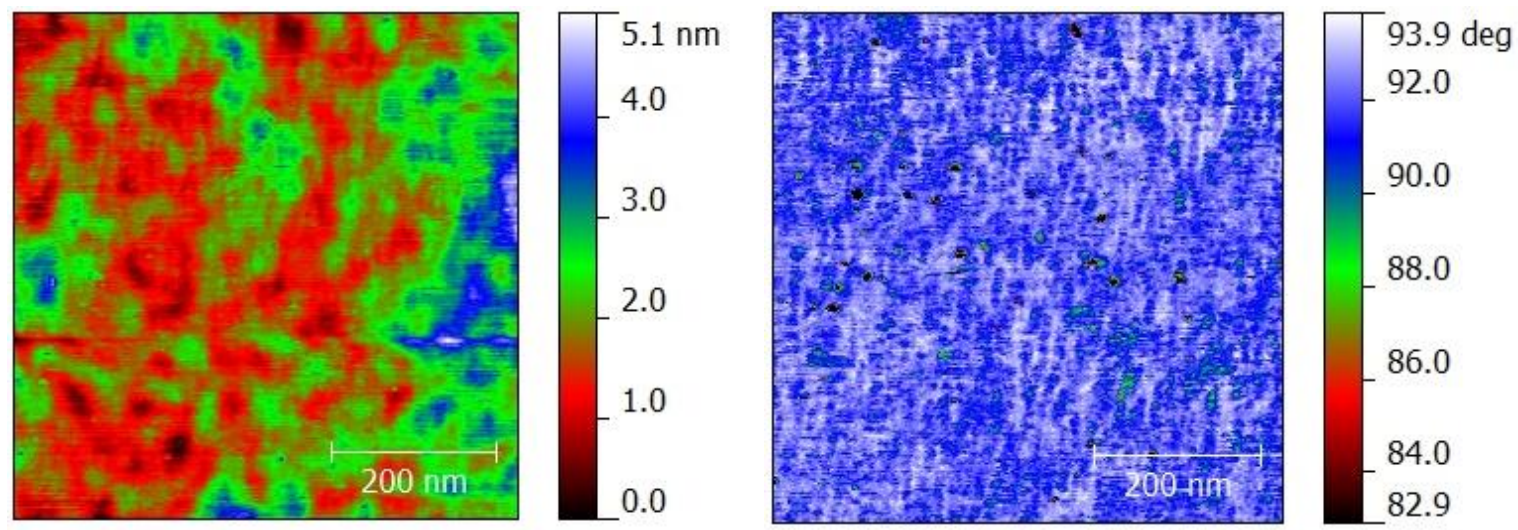

Figure 50: On the left, a topography scan of a PCPDTBT-P3HT:PCBM OPV annealed at $110^{\circ} \mathrm{C}$ for ten minutes. On the right, a phase angle scan of the same position and surface.

EFM further complicates the picture by now modeling both physical properties on the first pass, like topography and viscoelastic response, and electrical properties on the retrace, like conductivity and dielectric constant. (Figure 51). 

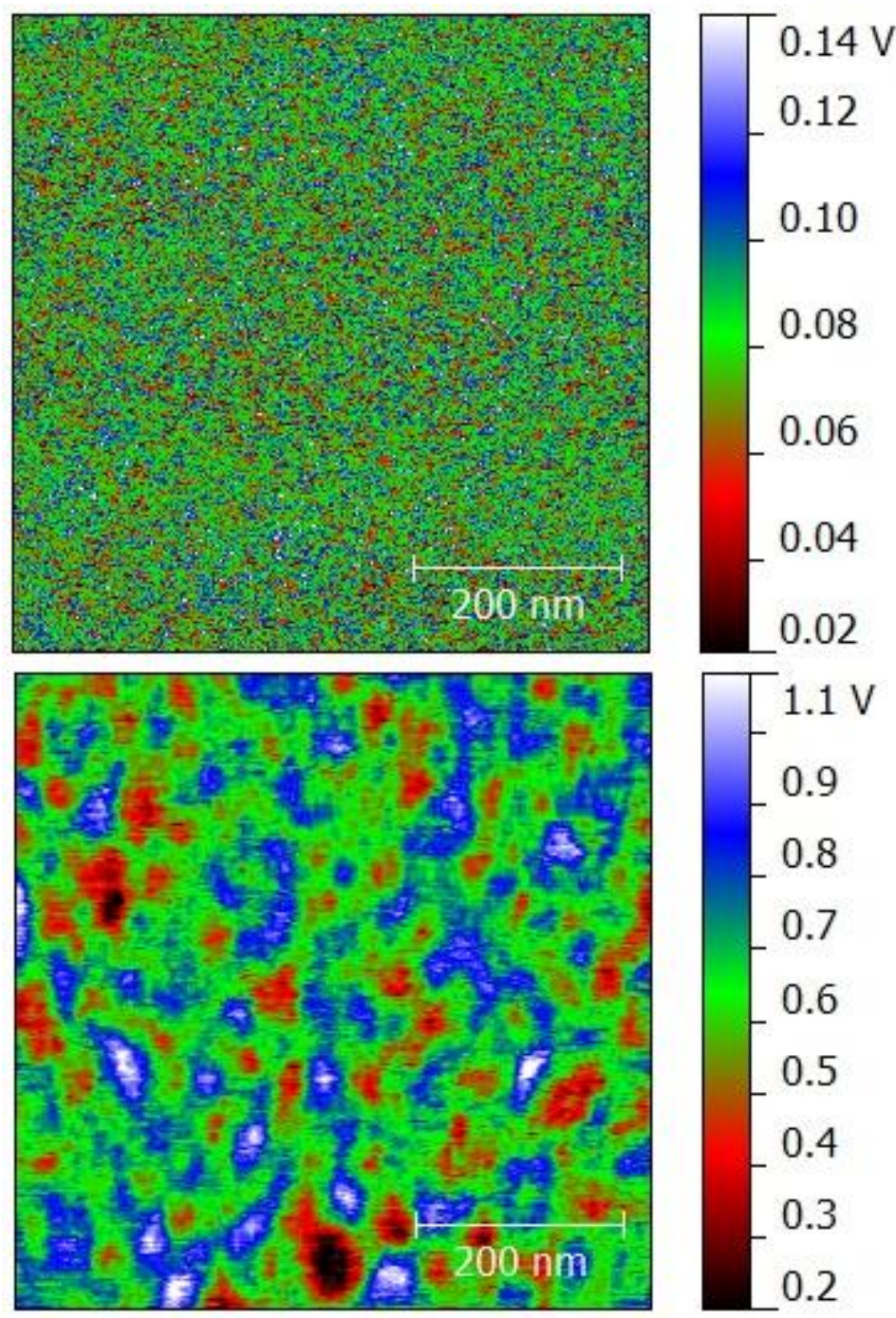

Figure 51: EFM phase angle scans of a PCPDTBT-P3HT:PCBM OPV annealed at $110^{\circ} \mathrm{C}$ for ten minutes: top showing normally distributed noise centered about $0.08 \mathrm{~V}$, bottom a $5 \mathrm{~V}$ bias clearly showing two distinctly different electric field responses with an intermediate response surrounding them.

The phase response was used rather than the height response because the phase response is more sensitive to changes in potential. ${ }^{66}$ It should be noted that the AFM tip used to collect these images was approximately $20 \mathrm{~nm}$ in diameter. Since the AFM tip diameter is nearly the same size as the expected morphology in a PCPDTBT-P3HT:PCBM BHJ, the collected phase angle EFM images should accurately represent the electrical domains of the BHJ morphology. 


\section{Data Analysis and Presentation}

The acquisition of EFM and AFM data is a complicated process. The saved files contain several "channels" that represent distinct aspects of the captured signal. As such, the captured data are often convoluted with scan errors, height disparity, and confusing scale representations. Thanks to powerful and intuitive image processing built specifically for AFM images, these errors can be fixed so that meaningful comparison can be made; however, careless image processing can introduce signal that is not indicative of reality. The data presented, both EFM and AFM, underwent median line height leveling, plane leveling, scar reduction, minimum data set to zero, and, most importantly, data tail removal. The AFM and EFM data collected generally follow some sort of normal or skewed normal distribution with a large portion of the data falling near the mean and a small portion falling well outside the majority. Data tail removal shifts the color scale to start and end where the majority of the observed data fall. Thus, the images presented do not start at zero, as zero did not fall in the majority of the data, and do not end at the absolute maximum observed. In other words, the data have been curated to better reflect the meaningful data, and exclude data that could distort opinion.

\section{Topography}

Spin coating is generally assumed to produce even, planarized films with little variation in surface thickness. The surface condition of the various OPV groups, both QD sensitized and control, shows an overall smooth film with small height disparities less than $\sim 250 \mathrm{~nm}$ in diameter with varying root mean square (RMS) roughness that increase with quantum dot loading (Figure 52, Figure 53, Figure 54). 

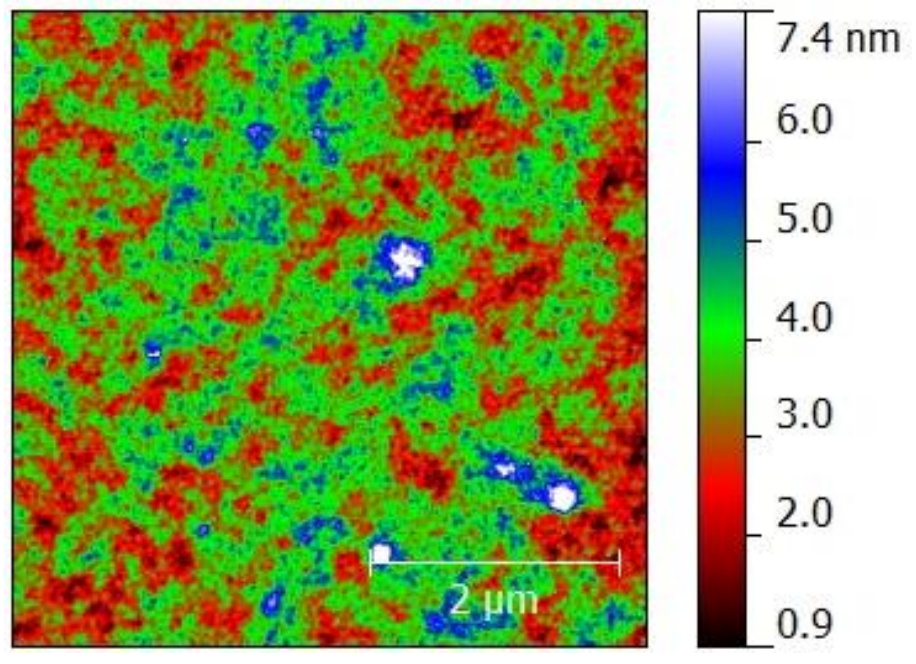

Figure 52: Topography scan of the PCPDTBT-P3HT:PCBM control annealed at $110^{\circ} \mathrm{C}$ for ten minutes, showing an overall smooth film and several small particles and an RMS value of $0.60 \mathrm{~nm}$.

The small particles were well adhered to the surface, as their location was constant and their presence did not disturb the phase of AFM tip oscillation. Considering that the particles were observed in the control group as well as the experimental group, it is likely that the particles are a result of small chunks of undissolved polymer deposited with the dissolved active layer materials.
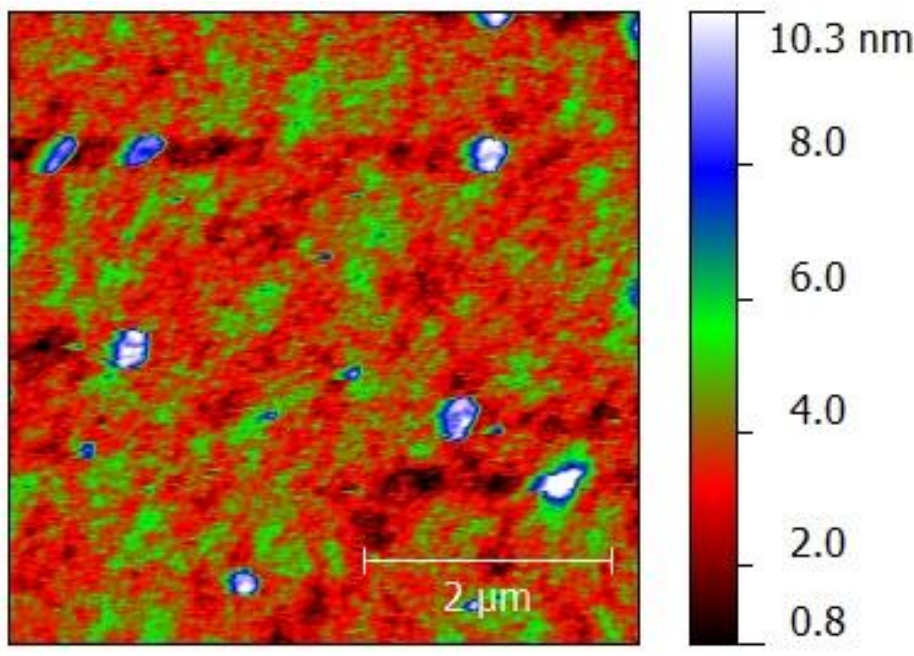

Figure 53: Topography scan of a PCPDTBT-P3HT:PCBM OPV sensitized with $0.25 \mathrm{mg} / \mathrm{mL}$ QDs and annealed at $110^{\circ} \mathrm{C}$ for ten minutes, showing increased RMS roughness of $0.81 \mathrm{~nm}$ and similar particles on the surface. 


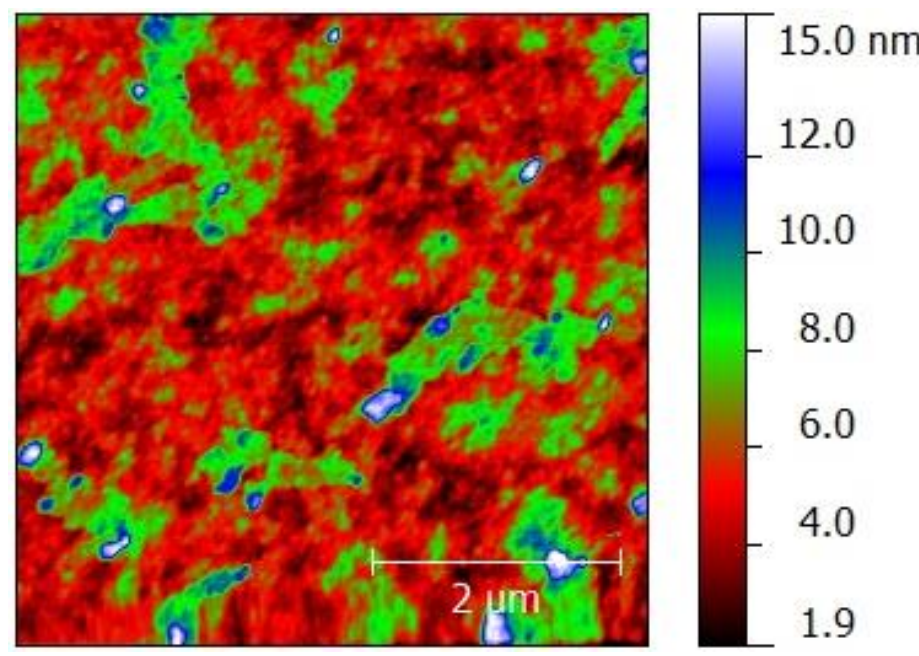

Figure 54: Topography scan of a PCPDTBT-P3HT:PCBM OPV sensitized with $1.0 \mathrm{mg} / \mathrm{mL}$ QDs and annealed at $110^{\circ} \mathrm{C}$ for ten minutes, showing further increased RMS roughness of $1.41 \mathrm{~nm}$ and similar particles on the surface.

\section{EFM Phase Analysis}

In the control group, the EFM phase responses are on the scale of polymer rich and

fullerene rich domains we would expect from an optimized BHJ (Figure 55).

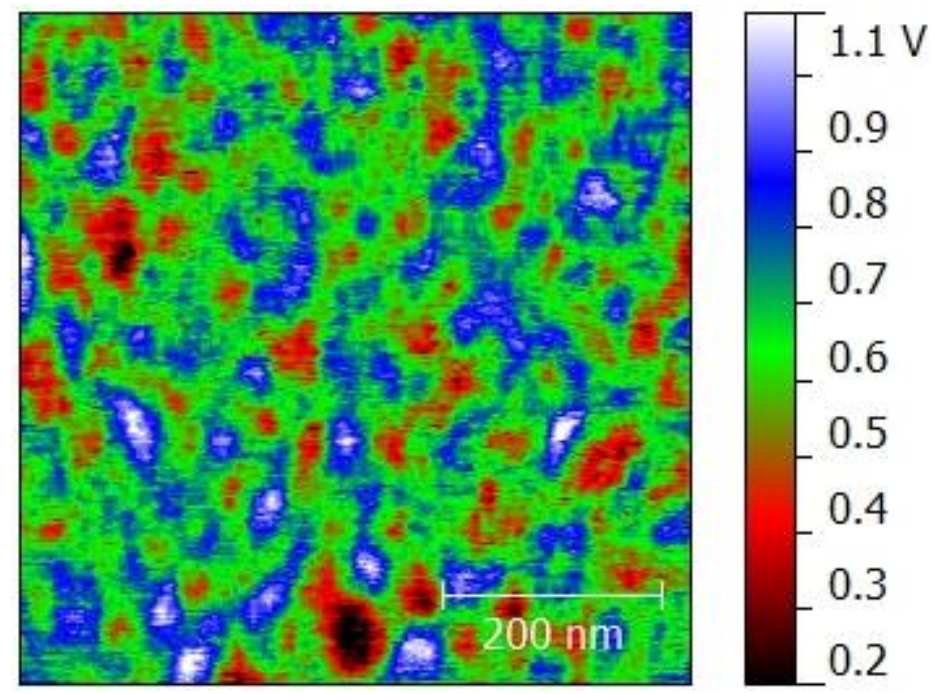

Figure 55: EFM phase image of the PCPDTBT-P3HT:PCBM control after annealing at $110^{\circ} \mathrm{C}$ for ten minutes which exhibits substantially different electric field strengths at sizes below $50 \mathrm{~nm}$ in diameter.

The addition of QDs has two effects: first, the maximum observed phase potential increases; second, the areas that exhibit either large or small potential are larger and occur in fewer spots (Figure 56). 

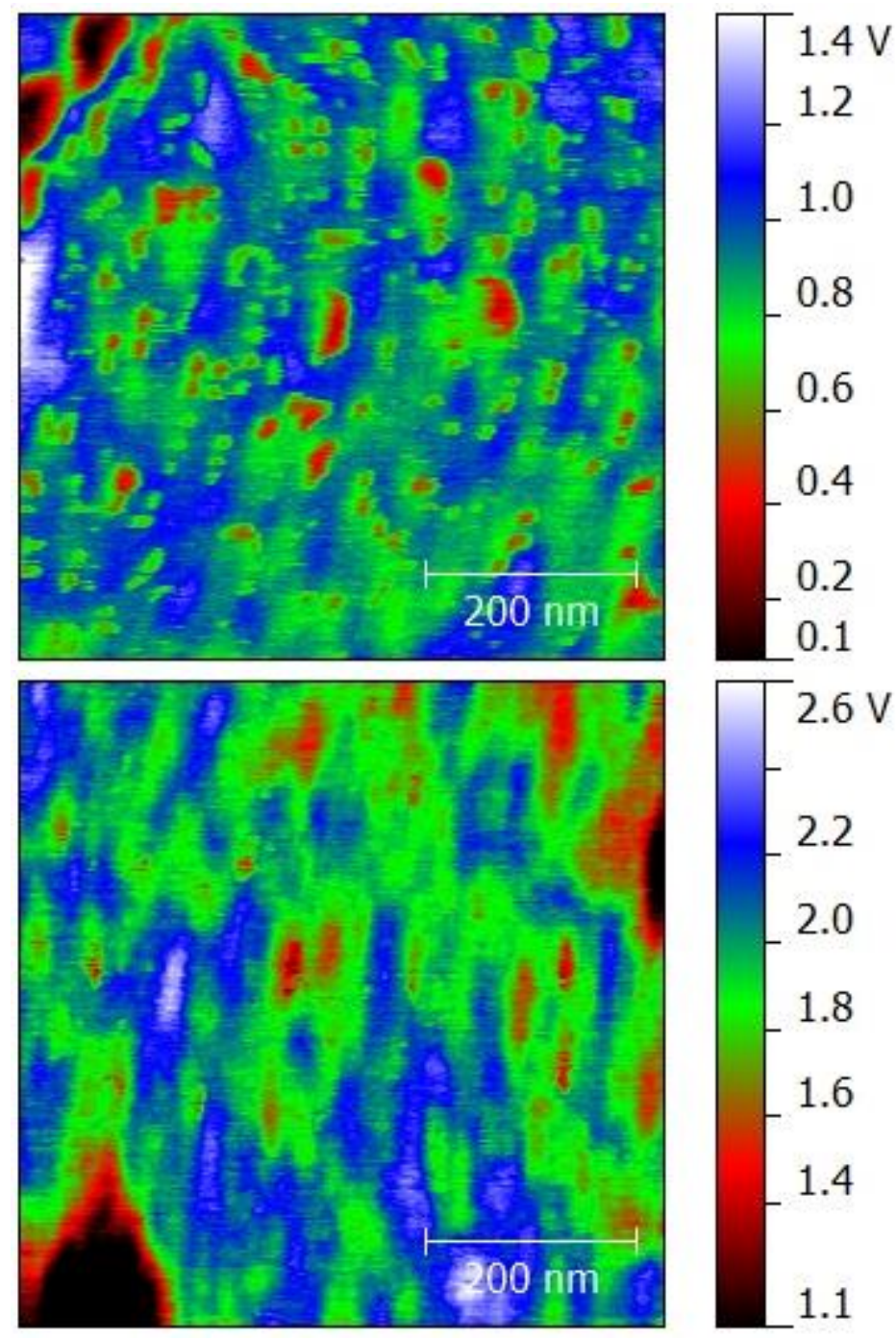

Figure 56: EFM phase images of OPVs sensitized with $0.25 \mathrm{mg} / \mathrm{mL}$ QDs (top) and $1.0 \mathrm{mg} / \mathrm{mL}$ (bottom) that exhibit increased phase potential differences and increased areas of electric field response when compare to the control.

The effect of increasing size for any one electric field response is poorer performance and increased recombination. The transitions between areas which can conduct the $5 \mathrm{~V}$ positive applied bias and those that cannot become larger and more diffuse with increasing QD concentration. 


\section{DISCUSSION}

It is a casual observation that the addition of QDs to further sensitize PCPDTBTP3HT:PCBM OPVs results in an overall poor performance. The more interesting aspect is why this decrease in performance occurred. In order to answer this question, we need to construct a model of how the addition of QD affects the solar cells that can explain the observed phenomena. Such a model would allow a deeper understanding of this particular material system as well as the results of this study, and ultimately will develop the conclusions to inform future work. The observed results are complex, with many different responses; however, when those results are combined with an in-depth understanding of OPV behavior, meaningful information can be extracted.

\section{The PCPDTBT-P3HT:PCBM Control}

To understand how the QDs change the OPVs, we must first understand the process the control undergoes. After the initial processing of the substrates, once the active layer has been applied, planarized, and patterned, the as-cast BHJ film is chaotic. The chlorobenzene solvent evaporates quickly in the dry nitrogen atmosphere of the glove box. Without the solvent, the donor polymers P3HT and PCPDTBT and the acceptor methanofullerene are no longer free to move, essentially frozen in place. Due to the speed of evaporation, the materials have no chance to rearrange into a more ordered, lower energy state. This active layer, while having an incredibly high interfacial area between donor and acceptor materials, is too disordered to easily allow free charge carriers to percolate through to their respective electrodes. The answer to the disorder is heat. Heating the device gives mobility to the polymers and PCBM allowing the polymer to diffuse and create polymer rich phases while the PCBM does the same. These newly formed "crystalline" regions create a pathway for free charge carriers to traverse the 
active layer, reach the electrodes, and be put to work. Unfortunately, free charge carriers are not the only charge carriers of interest. We also care about the excitons, coulombically bound electron-hole pairs. Since the dielectric constant of the active layer is low, negatively charged electrons excited by the absorption of light are not effectively screened from the positively charged hole left behind. The attraction between the two must be actively broken to create free charge carriers that can be used to produce work. This is where the BHJ comes in, because whenever an exciton reaches an interface between donor and acceptor material, the electron can lower its energy by transferring to the acceptor material. These excitons can only travel for so long without recombining, which means that there is a negative consequence for growing the polymer rich phases in the BHJ. Therefore, if the polymer rich phases are too small in the BHJ, free charge carriers face more resistance reaching the electrodes; however, if those phases are too large, a portion of the excitons generated from absorbing light will recombine before separating to become useful free charge carriers.

This behavior was precisely the behavior observed in the control devices. In the as-cast state, control devices exhibit poor $\mathrm{J}_{\mathrm{SC}}$, low FFs, and an average of $0.13 \%$ PCE. Once annealed to $110^{\circ} \mathrm{C}, \mathrm{J}_{\mathrm{SC}}$ increased four-fold, FFs improved, and PCE increased to eight times its previous level. A consequence of the increased PCBM crystallinity resulted in slightly lower $\mathrm{V}_{\mathrm{OC}}$, around a $1 \%$ loss. Annealing to higher temperatures, the creation of overly large polymer rich phases, suppressed device improvement and increased the drop in $\mathrm{V}_{\mathrm{OC}}$. 


\section{The Addition of Quantum Dots}

Quantum dots are small, 7-10 $\mathrm{nm}$ in diameter. Their smallness is what gives them the ability to tune their band-gap; however, small things have a large surface area to volume ratio that increases the energy associated with the solid. This small, high-energy site, serves as a nucleation point for $\mathrm{P} 3 \mathrm{HT}$ rich crystalline regions. It could serve as a nucleation site for any of the components of the BHJ; however, the only evidence of increased crystallinity was an increased "shoulder" response in the optical density, indicative of P3HT crystallinity, while no statistically significant drop in $\mathrm{V}_{\mathrm{OC}}$ was found indicating no significant increases in PCBM crystallinity. Because of the small increase in P3HT crystallinity, QD sensitized devices exhibited comparatively higher $\mathrm{J}_{\mathrm{SC}}$, decreased series resistance, and a PCE of $0.2 \%$ before annealing.

So the as-cast QD sensitized devices show higher crystallinity with QD concentration. If the QDs are acting as nucleation points for polymer to create a small amount of order, then the QD is lodged within the P3HT crystallites, complicating the extraction of free charge carriers. Given that QD devices exhibited poor FFs, higher series resistance, and less improvement of either after annealing, it is likely that the QDs are somehow affecting the extraction of charge. Since the QD is likely in the P3HT rich phase, further annealing would add P3HT to the crystallites distorted by the QD, meaning that the QD would further decrease device performance with increasing anneal temperature. 


\section{CONCLUSIONS}

The addition of QDs to further sensitize PCPDTBT-P3HT:PCBM OPVs to the solar spectrum resulted in an increased optical density and poor electrical performance. The added QDs served as nucleation points for the P3HT, which resulted in small crystallites surrounding QDs that are directly observable in the crystalline P3HT "shoulders" in the optical density measurements. The higher degree of crystallization in as-cast devices resulted in larger $\mathrm{J}_{\mathrm{SC}}$, and PCE with smaller series resistance. Since the QDs served as nucleation points for the P3HT, the typical performance increases from annealing were suppressed. The annealed QD sensitized devices showed smaller improvement in the $\mathbf{J}_{\mathrm{SC}}$ and similar final $\mathbf{J}_{\mathrm{SC}}$ values, lower FFs and, a smaller decrease in series resistance leading to a larger final series resistance. Although we would expect the $\mathrm{J}_{\mathrm{SC}}$ to also be smaller, a large temperature dependence in the final $\mathrm{J}_{\mathrm{SC}}$ value could effectively mask any observable trend. The addition of QDs and over annealing decreased $\mathrm{V}_{\mathrm{OC}}$ well beyond the decrease observed in the control devices. A decrease in hole mobility from unfavorable morphology created by the addition of QDs could increase the number of holes present in the HOMO level of the donor polymer, effectively decreasing the LUMO HOMO offset. Increased anneal temperature added more $\mathrm{P} 3 \mathrm{HT}$ to the crystallites containing QDs, meaning that more material responsible for hole transport was added to the unfavorable morphology, which compounds the effect on mobility and $\mathrm{V}_{\mathrm{OC}}$. Thus the addition of QDs to sensitize PCPDTBTP3HT:PCBM OPVs results in:

1. Increased optical density, and decreased electrical performance.

2. Quantum dots serving as nucleation points for P3HT crystallization.

3. Decrease in overall hole mobility within the P3HT due to QDs distorting the polymer crystal. 


\section{REFERENCES}

1. "Energy and Industrialization." OPEC Review 8.4 (1984): 409-33. Web.

2. "International Energy Statistics - EIA." International Energy Statistics - EIA. Energy Information Agency, n.d. Web. 02 Apr. 2014.

3. "U.S. Energy Information Administration - EIA - Independent Statistics and Analysis." International Energy Outlook 2013. Energy Information Agency, n.d. Web. 12 Apr. 2014.

4. "Members and Partners - OECD." Members and Partners - OECD. Organization for Co-operation and Development, n.d. Web. 13 Jan. 2014.

5. "World Energy Demand and Economic Outlook." United States of America. U.S. Energy Information Administration. Office of Energy Analysis. International Energy Outlook 2013 with Projections to 2040. S.1.: Energy Information Admini, 2013. Print.

6. "Energy-Related Carbon Dioxide Emissions" United States of America. U.S. Energy Information Administration. Office of Energy Analysis.International Energy Outlook 2013 with Projections to 2040. S.1.: Energy Information Admini, 2013. Print.

7. United States of America. U.S. Department of Energy. Office of Electricity Delivery \& Energy Reliability. Smart Grid. U.S. Department of Energy, n.d. Web. 01 June 2014.

8. NREL. "Levelized Cost of Energy Calculator." NREL: Energy Analysis -. NREL, n.d. Web. 03 June 2014. 
9. U.S. Energy Information Administration. "Levelized Cost and Levelized Avoided Cost of New Generation Resources in the Annual Energy Outlook 2014." U.S. Energy Information Administration. U.S. Energy Information Administration, 17 Apr. 2014. Web.

10. Luque, A., and Steven Hegedus. "Solar Grade Silicon Feedstock." Handbook of Photovoltaic Science and Engineering. Hoboken, NJ: Wiley, 2003. 153-204. Print.

11. Kasap, S. O. "Optical Absorption." Principles of Electronic Materials and Devices. 3rd ed. Boston: McGraw-Hill, 2006. 427-31. Print.

12. Shockley, William, and Hans J. Queisser. "Detailed Balance Limit of Efficiency of P-N Junction Solar Cells." Journal of Applied Physics 32.3 (1961): 510-19. Web.

13. Jain, Kanti, M. Klosner, and S. Raghunandan. "Flexible Electronics and Displays: High-Resolution, Roll-to-Roll, Projection Lithography and Photoablation Processing Technologies for High-Throughput Production." Flexible Electronics and Displays: High-Resolution, Roll-to-Roll, Projection Lithography and Photoablation Processing Technologies for High-Throughput Production 93.8 (2005): 1500-510. IEEE Xplore. IEEE. Web. 02 June 2014.

14. Brabec, Christoph J., Srinivas Gowrisanker, Jonathan J. M. Halls, Darin Laird, Shijun Jia, and Shawn P. Williams. "Polymer-Fullerene Bulk-Heterojunction Solar Cells." Advanced Materials 22.34 (2010): 3839-856. Web.

15. Inoue, Kanzan, Ross Ulbricht, Pallavi C. Madakasira, William M. Sampson, Sergey Lee, Jose Gutierrez, John Ferraris, and Anvar A. Zakhidov. "Temperature and 
Time Dependence of Heat Treatment of RR-P3HT/PCBM Solar Cell." Synthetic Metals 154.1-3 (2005): 41-44. Web.

16. Mandoc, M. M., L. J. A. Koster, and P. W. M. Blom. "Optimum Charge Carrier Mobility in Organic Solar Cells."Applied Physics Letters 90.13 (2007): 133504. Web.

17. Peumans, Peter, and Stephen R. Forrest. "Separation of Geminate Charge-pairs at Donor-acceptor Interfaces in Disordered Solids." Chemical Physics Letters 398.1-3 (2004): 27-31. Web.

18. Kim, J. Y., K. Lee, N. E. Coates, D. Moses, T.-Q. Nguyen, M. Dante, and A. J. Heeger. "Efficient Tandem Polymer Solar Cells Fabricated by All-Solution Processing." Science 317.5835 (2007): 222-25. Web.

19. Tipnis, Ritesh, Jan Bernkopf, Shinjun Jia, John Krieg, Sergey Li, Mark Storch, and David Laird. "Large-area Organic Photovoltaic Module—Fabrication and Performance." Solar Energy Materials \& Solar Cells 93 (2009): 442-46. Web.

20. Hauch, Jens A., Pavel Schilinsky, Stelios A. Choulis, Richard Childers, Markus Biele, and Christoph J. Brabec. "Flexible Organic P3HT:PCBM Bulkheterojunction Modules with More than 1 Year Outdoor Lifetime." Solar Energy Materials and Solar Cells 92.7 (2008): 727-31. Web.

21. Zhou, Yunfei, Michael Eck, Clemens Veit, Birger Zimmermann, Frank Rauscher, Phenwisa Niyamakom, Seyfullah Yilmaz, Ines Dumsch, Sybille Allard, and Ullrich Scherf. "Efficiency Enhancement for Bulk-heterojunction Hybrid Solar Cells Based on Acid Treated CdSe Quantum Dots and Low Bandgap Polymer 93 
PCPDTBT." Solar Energy Materials and Solar Cells 95.4 (2011): 1232-237.

Web.

22. Bullis, Kevin. "Quantum Dots Produce More Colorful Sony TVs | MIT Technology Review." MIT Technology Review. MIT Technology Review, 11 Jan. 2013. Web. 10 July 2014.

23. Carroll, Bradley W., and Dale A. Ostlie. "Blackbody Radiation." An Introduction to Modern Astrophysics. 2nd ed. San Francisco, Calif. ; Munich: Pearson/Addison Wesley, 2007. 68-71. Print.

24. Young, Hugh D., Roger A. Freedman, A. Lewis Ford, and Francis Weston Sears. "Scattering of Light." Sears and Zemansky's University Physics. 12th ed. Vol. 1. San Francisco: Pearson Addison Wesley, 2008. 1142-144. Print.

25. ASTM Standard G173, "Reference Solar Spectral Irradiances," ASTM International, West Conshohocken, 2008.

26. Kasap, S. O. "Elementary Quantum Physics." Principles of Electronic Materials and Devices. Boston: McGraw-Hill, 2006. 191-284. Print.

27. Brown, Ronald F. "Insulators and Semiconductors." Solid State Physics: An Introduction for Scientists and Engineers. San Luis Obispo, CA: El Corral Publications, 2001. 225-55. Print.

28. Klauk, Hagen. Organic Electronics: Materials, Manufacturing and Applications. Weinheim: Wiley-VCH, 2006. Print.

29. "Discover LG Premium Entertainment | LG USA." Discover LG Premium Entertainment | LG USA. N.p., n.d. Web. 10 July 2014 
30. Koezuka, H., A. Tsumura, and T. Ando. "Field-effect Transistor with Polythiophene Thin Film." Synthetic Metals 18.1-3 (1987): 699-704. Web.

31. "The Nobel Prize in Chemistry 2000." The Nobel Prize in Chemistry 2000. Nobel Media, n.d. Web. 02 June 2014.

32. Tang, C. W. "Two-layer Organic Photovoltaic Cell." Applied Physics Letters 48.2 (1986): 183. Web.

33. Silberberg, Martin S., and Elizabeth Bent. Weberg. "The Mode of Orbital Overlap and The Types of Covalent Bonds."Chemistry: The Molecular Nature of Matter and Change. 5th ed. Boston: McGraw-Hill, 2009. 418-22. Print.

34. Brédas, Jean-Luc, David Beljonne, Veaceslav Coropceanu, and Jérôme Cornil. "Charge-Transfer and Energy-Transfer Processes in $\pi$-Conjugated Oligomers and Polymers: A Molecular Picture." Chemical Reviews 104.11 (2004): 4971-5004. Web.

35. Cubane. "File:PCBM.png." Wikipedia. Wikimedia, 7 Nov. 2009. Web.

36. Ma, W., C. Yang, X. Gong, K. Lee, and A. J. Heeger. "Thermally Stable, Efficient Polymer Solar Cells with Nanoscale Control of the Interpenetrating Network Morphology." Advanced Functional Materials 15.10 (2005): 1617-622. Web.

37. Chamberlain, G.a. "Organic Solar Cells: A Review." Solar Cells 8.1 (1983): 47-83. Web.

38. Koppe, Markus, Hans-Joachim Egelhaaf, Gilles Dennler, Markus C. Scharber, Christoph J. Brabec, Pavel Schilinsky, and Claudia N. Hoth. "Near IR Sensitization of Organic Bulk Heterojunction Solar Cells: Towards Optimization 
of the Spectral Response of Organic Solar Cells." Advanced Functional Materials 20.2 (2010): 338-46. Web.

39. Halls, J. J. M., K. Pichler, R. H. Friend, S. C. Moratti, and A. B. Holmes. "Exciton Diffusion and Dissociation in a Poly(p-phenylenevinylene)/C60 Heterojunction Photovoltaic Cell." Applied Physics Letters 68.22 (1996): 3120. Web.

40. Sigma Aldrich. "Poly(3-hexylthiophene-2,5-diyl)." P3HT. Sigma Aldrich, n.d. Web. 03 June 2014.

41. Sigma Aldrich. "PCPDTBT." AldrichCPR, Mw 7,000-20,000. Sigma Aldrich, n.d. Web. 03 June 2014.

42. Flanagan, D. "File:Polythiophenes Pedotpss.png." Wikipedia. Wikimedia, 15 Nov. 2012. Web.

43. Morana, Mauro, Matthias Wegscheider, Alberta Bonanni, Nikos Kopidakis, Sean Shaheen, Markus Scharber, Zhengguo Zhu, David Waller, Russell Gaudiana, and Christoph Brabec. "Bipolar Charge Transport in PCPDTBT-PCBM BulkHeterojunctions for Photovoltaic Applications." Advanced Functional Materials 18.12 (2008): 1757-766. Web.

44. Gong, Xiong, Minghong Tong, Fulvio G. Brunetti, Junghwa Seo, Yanming Sun, Daniel Moses, Fred Wudl, and Alan J. Heeger. "Bulk Heterojunction Solar Cells with Large Open-Circuit Voltage: Electron Transfer with Small Donor-Acceptor Energy Offset." Advanced Materials 23.20 (2011): 2272-277. Web.

45. Halls, J., J. Cornil, D. Dos Santos, R. Silbey, D.-H. Hwang, A. Holmes, J. Brédas, and R. Friend. "Charge- and Energy-transfer Processes at Polymer/polymer 
Interfaces: A Joint Experimental and Theoretical Study." Physical Review B 60.8(1999): 5721-727. Web.

46. Alvarado, S., P. Seidler, D. Lidzey, and D. Bradley. "Direct Determination of the Exciton Binding Energy of Conjugated Polymers Using a Scanning Tunneling Microscope." Physical Review Letters 81.5 (1998): 1082-085. Web.

47. Alivisatos, A. P. "Semiconductor Clusters, Nanocrystals, and Quantum Dots." Science 271.5251 (1996): 933-37. Web.

48. Murray, C. B., D. J. Norris, and M. G. Bawendi. "Synthesis and Characterization of Nearly Monodisperse CdE (E = Sulfur, Selenium, Tellurium) Semiconductor Nanocrystallites." Journal of the American Chemical Society115.19 (1993): 8706715. Web.

49. Dubertret, B. "In Vivo Imaging of Quantum Dots Encapsulated in Phospholipid Micelles." Science 298.5599 (2002): 1759-762. Web.

50. Dabbousi, B. O., J. Rodriguez-Viejo, F. V. Mikulec, J. R. Heine, H. Mattoussi, R. Ober, K. F. Jensen, and M. G. Bawendi. "(CdSe)ZnS Core-Shell Quantum Dots: Synthesis and Characterization of a Size Series of Highly Luminescent Nanocrystallites." The Journal of Physical Chemistry B 101.46 (1997): 9463-475. Web.

51. Kim, Sungjee, Brent Fisher, Hans-Jürgen Eisler, and Moungi Bawendi. "Type-II Quantum Dots: CdTe/CdSe(Core/Shell) and CdSe/ZnTe(Core/Shell) Heterostructures." Journal of the American Chemical Society 125.38 (2003): 11466-1467. Web. 
52. Kim, Sungjee, and Moungi G. Bawendi. "Oligomeric Ligands for Luminescent and Stable Nanocrystal Quantum Dots."Journal of the American Chemical Society 125.48 (2003): 14652-4653. Web.

53. Kasap, S. O. "Solar Cells." Principles of Electronic Materials and Devices. 3rd ed. Boston: McGraw-Hill, 2006. 551-64. Print.

54. Brown, Ronald F. "Semiconductor Devices." Solid State Physics: An Introduction for Scientists and Engineers. San Luis Obispo, CA: El Corral Publications, 2001. 256-312. Print.

55. Tsoi, Wing C., Steve J. Spencer, Li Yang, Amy M. Ballantyne, Patrick G. Nicholson, Alan Turnbull, Alex G. Shard, Craig E. Murphy, Donal D. C. Bradley, Jenny Nelson, and Ji-Seon Kim. "Effect of Crystallization on the Electronic Energy Levels and Thin Film Morphology of P3HT:PCBM Blends." Macromolecules 44.8 (2011): 2944-952. Web.

56. Brabec, C. J., A. Cravino, D. Meissner, N. S. Sariciftci, T. Fromherz, M. T. Rispens, L. Sanchez, and J. C. Hummelen. "Origin of the Open Circuit Voltage of Plastic Solar Cells." Advanced Functional Materials 11.5 (2001): 374-80. Web.

57. Günes, Serap, Helmut Neugebauer, and Niyazi Serdar Sariciftci. "Conjugated Polymer-Based Organic Solar Cells."Chemical Reviews 107.4 (2007): 1324-338. Web.

58. Mihailetchi, V. D., H. X. Xie, B. De Boer, L. J. A. Koster, and P. W. M. Blom. "Charge Transport and Photocurrent Generation in Poly(3-hexylthiophene): Methanofullerene Bulk-Heterojunction Solar Cells." Advanced Functional Materials 16.5 (2006): 699-708. Web. 
59. Dennler, Gilles, Markus C. Scharber, and Christoph J. Brabec. "Polymer-Fullerene Bulk-Heterojunction Solar Cells."Advanced Materials 21.13 (2009): 1323-338. Web.

60. Khodabakhsh, S., B. M. Sanderson, J. Nelson, and T. S. Jones. "Using SelfAssembling Dipole Molecules to Improve Charge Collection in Molecular Solar Cells." Advanced Functional Materials 16.1 (2006): 95-100. Web.

61. Zhang, F. L., A. Gadisa, O. Inganäs, M. Svensson, and M. R. Andersson. "Influence of Buffer Layers on the Performance of Polymer Solar Cells." Applied Physics Letters 84.19 (2004): 3906. Web.

62. Heraeus: Conductive Polymers Division. "CLEVIOSTM P VP AI 4083." CLEVIOS ${ }^{\mathrm{TM}}$ P VP AI 4083 (2010): n. pag. Heraeus, 22 Dec. 2010. Web.

63. Madsen, Morten V., Thomas Tromholt, Arvid Böttiger, Jens W. Andreasen, Kion Norrman, and Frederik C. Krebs. "Influence of Processing and Intrinsic Polymer Parameters on Photochemical Stability of Polythiophene Thin Films." Polymer Degradation and Stability 97.11 (2012): 2412-417. Web.

64. "Ocean Optics - USB2000+ Fiber Optic Spectrometer.” Ocean Optics USB200+ Fiber Optic Spectrometer. N.p., n.d. Web. 10 July 2014.

65. Morita, S. Roadmap of Scanning Probe Microscopy. Berlin: Springer, 2007. Print.

66. Lei, C. H., A. Das, M. Elliott, and J. E. Macdonald. "Quantitative Electrostatic Force Microscopy-phase Measurements." Nanotechnology 15.5 (2004): 627-34. Web. 